INTERNATIONAL MONETARY FUND

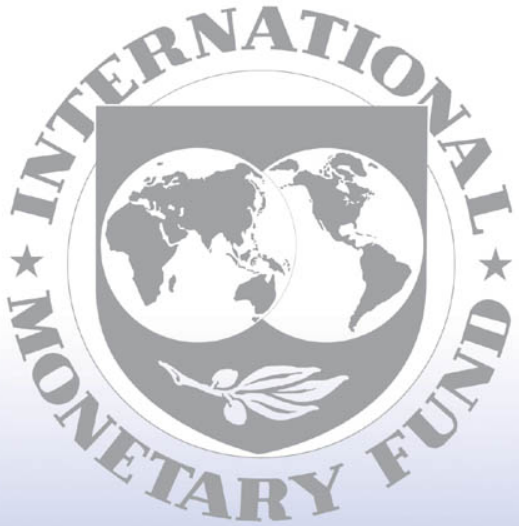

Staff

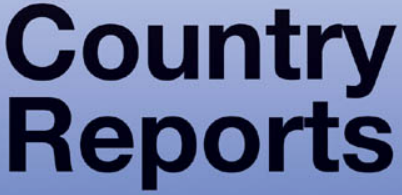




\section{Senegal: Third and Fourth Reviews Under the Three-Year Arrangement Under the Poverty Reduction and Growth Facility and Request for Waiver of Performance Criteria-Staff Report; Press Release on the Executive Board Discussion; and Statement by the Executive Director for Senegal}

In the context of the third and fourth reviews under the three-year arrangement under the Poverty Reduction and Growth Facility and a request for a waiver of performance criteria, the following documents have been released and are included in this package:

- $\quad$ the staff paper for the Third and Fourth Reviews Under the Three-Year Arrangement Under the Poverty Reduction and Growth Facility and Request for Waiver of Performance Criteria, prepared by a staff team of the IMF, following discussions that ended on December 16, 2006, with the officials of Senegal on economic developments and policies. Based on information available at the time of these discussions, the staff report was completed on December 29, 2006. The views expressed in the staff report are those of the staff team and do not necessarily reflect the views of the Executive Board of the IMF.

- $\quad$ a Press Release summarizing the views of the Executive Board as expressed during its January 9, 2006 discussion of the staff report that completed the request and reviews.

- $\quad$ a statement by the Executive Director for Senegal.

The documents listed below have been or will be separately released.

Letter of Intent sent to the IMF by the authorities of Senegal*

Memorandum of Economic and Financial Policies by the authorities of Senegal*

*Also included in Staff Report

The policy of publication of staff reports and other documents allows for the deletion of market-sensitive information.

To assist the IMF in evaluating the publication policy, reader comments are invited and may be sent by e-mail to publicationpolicy@imf.org.

Copies of this report are available to the public from

International Monetary Fund • Publication Services

$70019^{\text {th }}$ Street, N.W. • Washington, D.C. 20431

Telephone: (202) 623-7430 • Telefax: (202) 623-7201

E-mail: publications@imf.org • Internet: http://www.imf.org

Price: $\$ 15.00$ a copy

International Monetary Fund

Washington, D.C. 
This page intentionally left blank

(C)International Monetary Fund. Not for Redistribution 


\section{INTERNATIONAL MONETARY FUND}

\section{SENEGAL}

\section{Third and Fourth Reviews Under the Three-Year Arrangement Under the Poverty Reduction and Growth Facility and Request for Waiver of Performance Criteria}

Prepared by the African Department

(In consultation with other departments)

Approved by Saul Lizondo and Adnan Mazarei

December 29, 2005

- Senegal has enjoyed political stability in recent years. The civil strife in the Casamance region was resolved with the signing of a cease-fire agreement in January 2005. Presidential and parliamentary elections are scheduled for 2007.

- Senegal's three-year PRGF arrangement in an amount equivalent to SDR 24.27 million (15 percent of quota) was approved on April 28, 2003.

- The last Article IV consultation and the second review of the PRGF-supported program were completed in March 2005, and Senegal reached its completion point under the enhanced HIPC Initiative on April 19, 2004.

- The Executive Board has determined that Senegal meets the qualification criteria for receiving debt relief under the Multilateral Debt Relief Initiative (MDRI) and that the Fund is prepared to approve MDRI relief for Senegal as soon as the remaining consents of the contributors to the PRGF Trust Subsidy Account are received.

- In the attached letter of intent and memorandum on economic and financial policies (MEFP) dated December 29, 2005 (Appendix I), the Minister of Economy and Finance reviews progress during 2005, and outlines the government's economic program and policies for 2006.

- Discussions for the third and fourth reviews of the PRGF-supported program were held in Dakar (June 3-17 and December 9-16, 2005) and Washington (June 27-July 1 and September 22-29, 2005). The principal Senegalese representatives included Mr. Diop, Minister of Economy and Finance, Mr. Soumaré, Minister of Budget, and Mr. Ndiaye, National Director of the BCEAO. Staff also met with the Prime Minister, Mr. Sall, the Minister of Energy, Mr. Niang, and representatives of the diplomatic community, NGOs, trade unions, the business community, and international institutions. The staff team participating in one or more of the missions comprised Mr. Vaez-Zadeh (head), Ms. Pattillo, and Messrs. Doré, Josz, Krichene, Roudet (all AFR), and Segura-Ubiergo (FAD). Mr. Morisset (World Bank) participated in the December 2005 mission, and Mr. Sembene, Advisor to the Executive Director for Senegal, attended some of the meetings. 


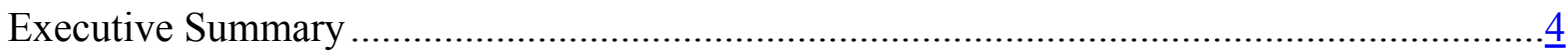

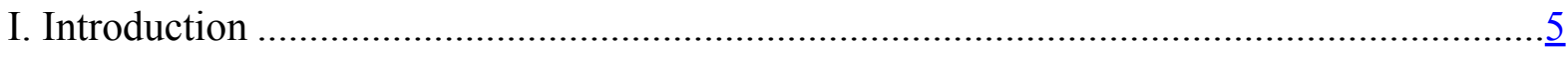

II. Economic Developments and Program implementation During 2004 and the

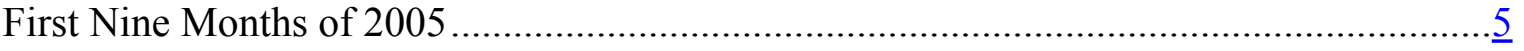

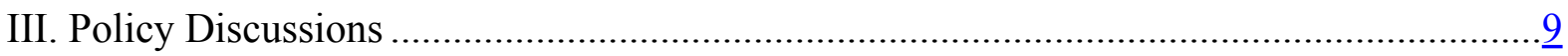

A. The Macroeconomic Outlook for 2006 ..............................................................

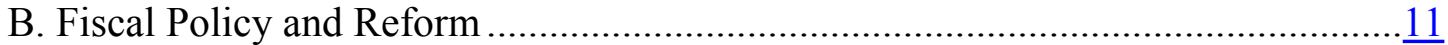

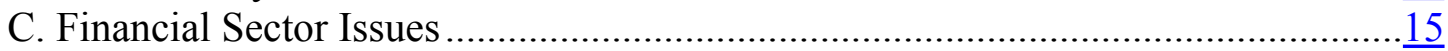

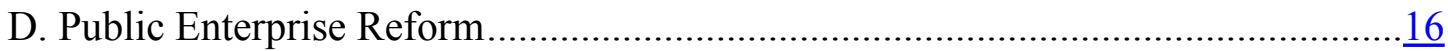

E. Relations with the Fund..............................................................................

F. Statistical Issues and Disclosure.....................................................................

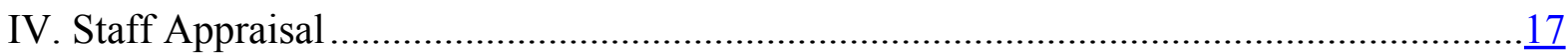

Boxes

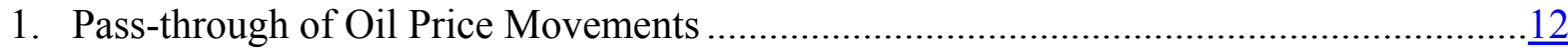

Figures

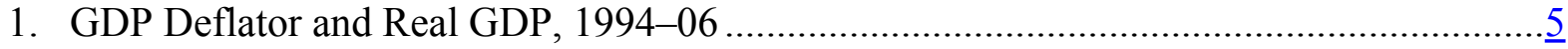

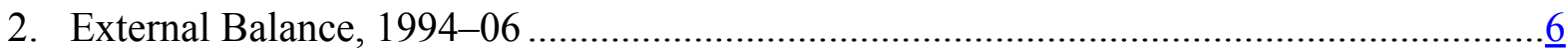

3. Exports of Goods and Workers' Remittances 1994-06.....................................................

4. Terms of Trade, Export and Relative Prices, and Effective Exchange Rates, 1994-September 2005

5. WAEMU: Average Real Effective Exchange Rates, 1994-September 2005 .......................7

6. Government Revenue and Expenditure 2000-06 …...................................................

7. Credit to the Economy and Banks' Excess Reserves, March 2000-September 05 .............. $\frac{8}{8}$

8. Progress Toward Millennium Development Goals, 1990-15 ........................................10

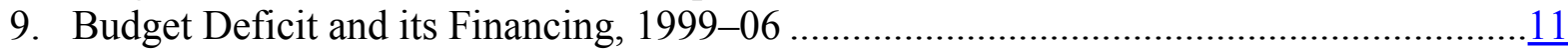

Tables

1. Quantitative Performance Criteria and Indicative Targets for 2005 ….............................19

2. Prior Actions, Structural Performance Criteria and Benchmarks for the Program under

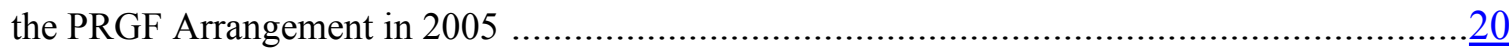

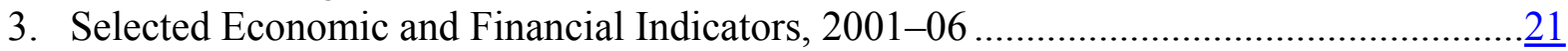

4. Savings-Investment Balances and National Accounts 2001-06.....................................22

5. Government Financial Operations, 2001-06 (in billions of CFA francs) ..........................23

6. Government Financial Operations, 2001-06 (in percent of GDP) ...................................24

7. Quarterly Government Financial Operations, 2005 …................................................. 


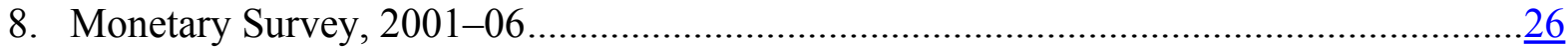

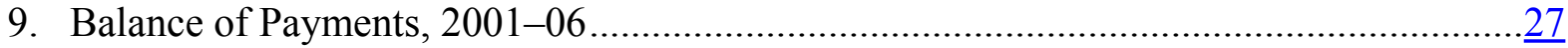

10. Selected Medium-Term Economic and Financial Indicators, 2003-10............................28

11. Medium-Term Balance of Payments, 2003-10 …….....................................................29

12. Fund Position During the Period of the PRGF Arrangement, 2003-06 ………................

13. Financial Soundness Indicators for the Banking Sector, 2000-05 ...................................

14. Schedule of Projected Reviews and Disbursements Under the PRGF

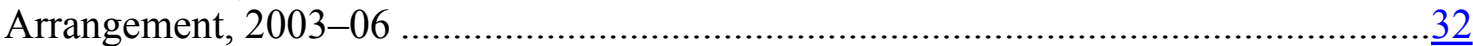

15. Millennium Development Goals ...........................................................................

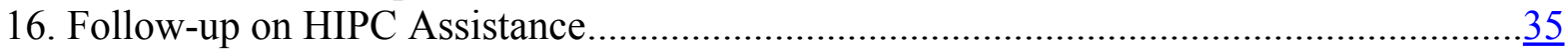

Appendices

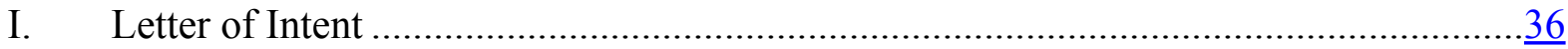

Attachment: Memorandum of Economic and Financial Policies ............................ $\frac{38}{2}$

Annex I Priority Measures for Improving Fiscal Transparency in 2005-06 … $\underline{\underline{52}}$

Annex II: Decree on Transparency Procedures for the International Airport

Blaise Diagne de Ndiass ..............................................................................

Annex III: Priority Measures for Enhancing the Performance of the

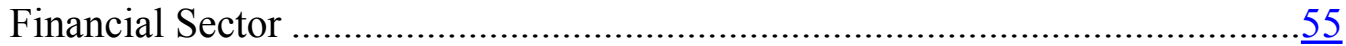

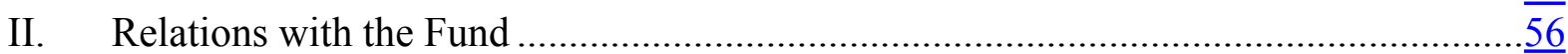

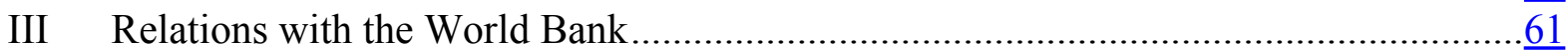

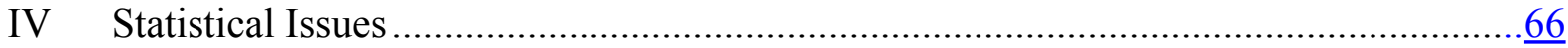




\section{EXECUTIVE SUMMARY}

Program implementation in 2005 was uneven. Most performance criteria (PCs) for the third and fourth reviews were met, except for the continuous PCs on the arrears of, and budgetary transfers to, the state electricity company, and the ceiling on no-bid public contracts (Table 1). On the basis of the corrective actions taken and planned, the authorities requested waivers for the missed PCs. The end-December 2005 PC on elimination of a protective tax for SONACOS was respected, but a new protective tax was imposed in December 2005. The other structural PC and four of the five structural benchmarks for 2005 were also met, two with some delay (Table 2).

Macroeconomic performance was strong in $\mathbf{2 0 0 4}$ and 2005, although slightly weaker than programmed. The program's growth and inflation objectives for 2004 were achieved and are expected to have been broadly attained in 2005. The overall fiscal deficit (including grants) more than doubled to 3.3 percent of GDP in 2004, and rose slightly further in 2005 . The deficits were overfinanced by external borrowing and debt relief in both years. The external current account deficit (including grants) is estimated to have widened by about one percentage point of GDP to 7.6 percent of GDP in 2005.

Economic prospects for $\mathbf{2 0 0 6}$ are for continued strong growth and low inflation. The projected overall budget deficit of 4 percent will not endanger macroeconomic stability, given the relatively low level of public debt (50 percent of GDP before the Multilateral Debt Relief Initiative (MDRI) - and mostly on concessional terms) and limited domestic borrowing planned for 2006. The external current account deficit is envisaged to remain broadly unchanged as percentage of GDP.

Fiscal transparency and governance will be enhanced. In particular, the use of the revenues from the new air travel tax — earmarked for the construction of a new airport—will be subject to transparency and accountability procedures; the procurement framework will be strengthened with the help of the World Bank; and the government's financial operations table - the main source of information on budget execution - will be revised to improve the transparency of fiscal outcomes.

The financial system is generally sound but burdened by a lingering high share of nonperforming loans, credit concentration, and an inadequate regulatory and judicial framework. The authorities have prepared an action plan to address these issues and, in particular, ensure easier seizure of collateral and better accounting practices by firms.

The monitoring of enterprises in which the government has a significant stake should be strengthened to enhance the control of the government's contingent liabilities. In particular, the financial problems of the ICS (a chemical company), one of Senegal's largest companies, should be addressed urgently as they could impose costs on the budget and pose a risk to the soundness of banks.

The authorities would like to establish a nonfinancial arrangement with the Fund on the expiry of the PRGF arrangement in April 2006. 


\section{INTRODUCTION}

1. Executive Board consideration of the third review of the program was delayed owing mainly to ongoing discussions of transparency procedures for the implementation of Dakar's new airport project. These discussions were completed in December 2005, when information for the test date for the fourth review (endSeptember 2005) also became available, making it possible to combine the third and fourth reviews.

2. Senegal has enjoyed high growth and low inflation in recent years (Figure 1 and Table 3). It has registered one of the lowest inflation rates in the West African Economic and Monetary Union (WAEMU) region since 1997. The PRGF arrangement aims at safeguarding these gains, while fostering an environment conducive to private sector-led growth and poverty reduction. Fiscal transparency and structural reforms constitute the two critical pillars of the authorities' economic program.

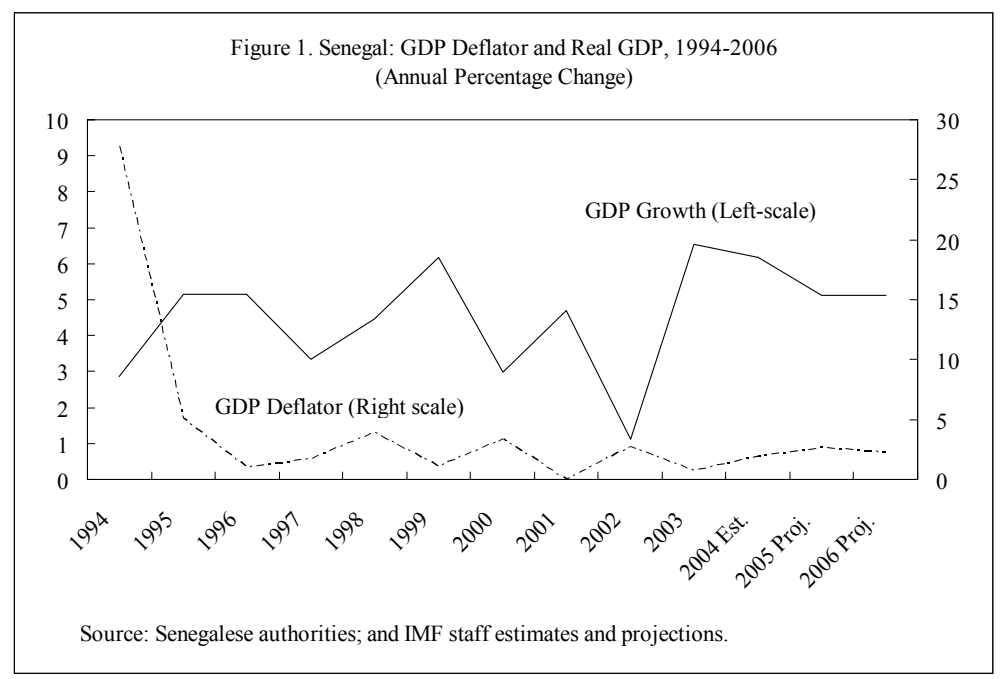

\section{Economic Developments And Program Implementation During 2004 AND THE FIRST NINE MONTHS OF 2005}

3. Macroeconomic performance in 2004 and 2005 was broadly in line with program projections, with robust growth and low inflation in both years. However, real GDP growth in 2005 was lower than the program target by about one percentage point (Table 4), mainly due to the increase in world oil prices.

4. The balance of payments is estimated to have deteriorated slightly in 2005 . The overall balance was in surplus in 2004, owing to strong inflow of private remittances and external assistance, but is estimated to have turned to a deficit in 2005 as the current account deficit widened with the rise in oil prices (Table 9, Figures 2 and 3).

5. The real effective exchange rate (REER) remained relatively stable in 2004, but depreciated slightly in the first nine months of 2005, reflecting mainly the depreciation of the euro against the U.S. dollar (Figure 4). Since 1994 (when the CFA franc was devalued), Senegal's REER has depreciated by about 12 percent, in contrast to an appreciation of the REER in almost all other countries of the WAEMU (Figure 5). 

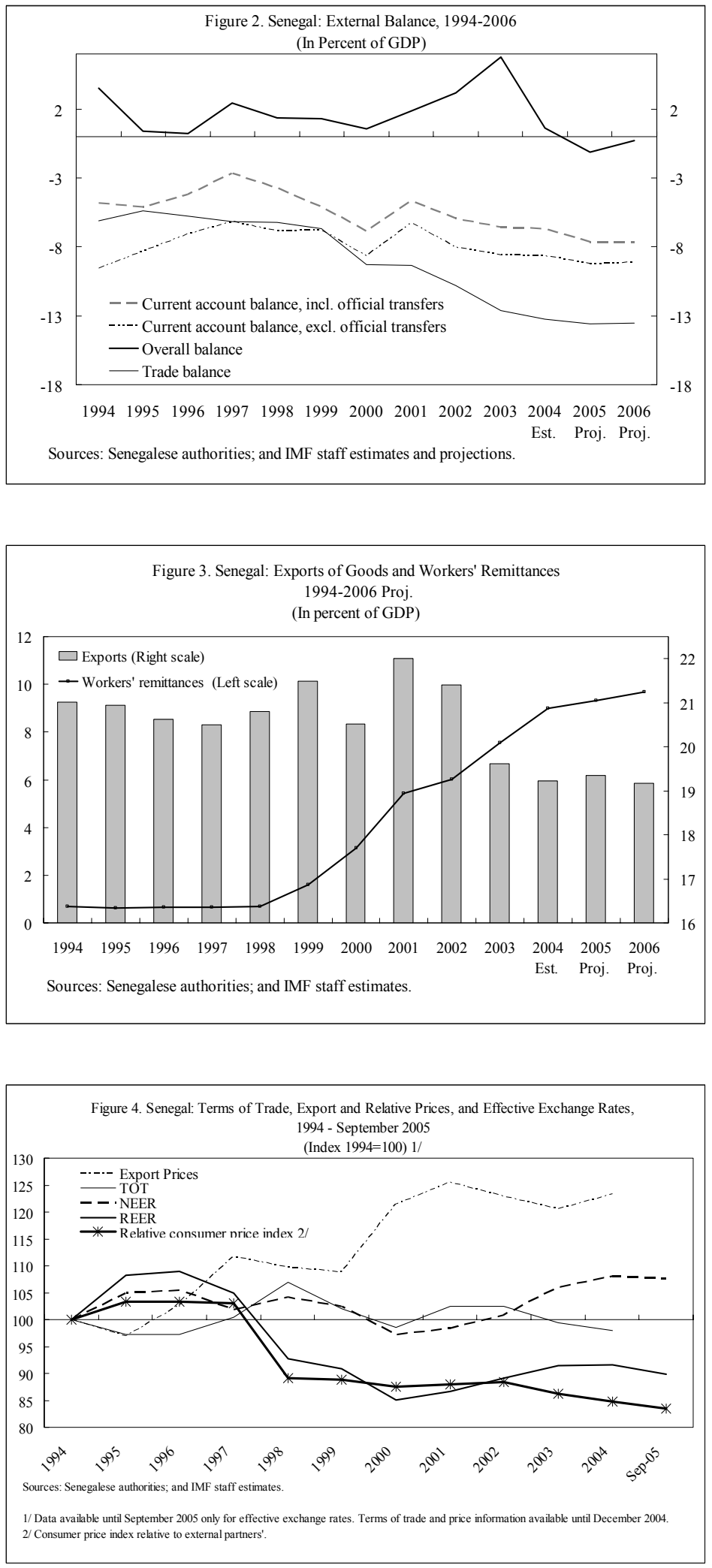

(C) International Monetary Fund. Not for Redistribution 
6. Fiscal performance in $\mathbf{2 0 0 4}$ and 2005 was in line with program targets (Tables 5, 6, and 7), ${ }^{1}$ with the basic fiscal surplus higher than programmed in the first nine months of 2005. The overall deficit is estimated to have increased slightly to 3.5 percent of GDP in 2005, but was over-financed through external resources (concessional loans). ${ }^{2}$ The government's net position vis-à-vis the banking sector improved.

\section{Revenue performance} remained strong in 2004 and further improved in the first three quarters of 2005, owing to the higher revenues from corporate income tax and oil-related taxes (Figure 6). ${ }^{3}$ In April 2005, a new tax on air travel was introduced by Presidential decree to finance construction of Dakar's new airport. The full year yield from this tax is estimated at 0.3 percent of GDP.

\section{Total expenditure} increased by about one percent of GDP in both 2004 and 2005, reflecting the growth of capital outlays and current spending, respectively. The growth of spending in 2005 was mainly due to higher transfers to the electricity company (SENELEC) and the oil refinery (SAR), estimated at 1.3 percent of GDP in 2005, to compensate them for insufficient increases in electricity rates and butane gas prices. The wage bill also rose substantially in 2005 as the three-year recruitment program came close to completion.

\footnotetext{
${ }^{1}$ The discussion of fiscal developments is based on the revised Table on Government Financial Operations (TOFE). The changes are explained in the MEFP, Box 1.

${ }^{2}$ Official flows of loans, grants, and debt relief have averaged 7 percent of GDP per year since the 1994 devaluation.

${ }^{3}$ Customs revenues weakened in 2004, as the share of zero- and low-tariff imports in total has increased, but strengthened in the first nine months of 2005 as customs administration improved and oil prices increased.
} 
9. On the monetary front, credit to the economy increased substantially in 2005, as short-term credit to oil suppliers and the chemical company (ICS) grew markedly (Table 8). Banks' excess reserves have trended downward since mid2004, but, at about 50 percent of required reserves, remained large at end-September 2005 (Figure 7). The BCEAO has tried to tailor reserve requirements to the liquidity situation in each WAEMU country, but it has not completely absorbed excess liquidity in the region (and in Senegal, in particular). As a result, banks' interest rates have been largely unaffected by changes in the BCEAO's lending rates, and remained broadly unchanged in 2005 .

\section{Staff projections indicate that the ratio of the NPV of debt to exports would} decline to about 116 percent by end-2005, following enhanced HIPC Initiative debt relief. The authorities have intensified contacts with all creditors with a view to increasing the rate of participation in the Enhanced HIPC Initiative and securing greater debt relief (Table 16). The ratio would fall to 72 percent after MDRI debt relief.

11. Program implementation during 2005 was mixed. Most of the quantitative performance criteria were met (Table 1). However, owing to the rapid increase in oil prices in 2005, the financial situation of the electricity company SENELEC deteriorated and the continuous PCs on arrears of, and budgetary transfers to, the company were missed. The performance criterion on no-bid public contracts was also exceeded at end-September. The authorities explained that the strategic nature of a few large contracts (digitized identification cards for the next election and military equipment) prevented recourse to the usual tender procedures. However, they agreed with staff that the excessive use of no-bid contracts was a recurring problem.

\section{Staff supports the government's request for a waiver for the nonobservance of} the three PCs, in light of the authorities' explanations and the remedial actions already taken and envisaged (MEFP, paras. 7 and 9). These include: (i) the decision to fully adjust electricity prices to oil price increases by end-2006, in accordance with the new marketoriented electricity price formula adopted in October 2005; (ii) repayment of the arrears by SENELEC; (iii) remedial actions taken by SENELEC to improve its profitability; and (iv) measures to improve procurement practices. 
13. Compliance with structural conditionality was not fully satisfactory. One structural PC and four of the five structural benchmarks were met, two with some delay (Table 2). The only structural benchmark that was not met (improved presentation of the main fiscal table) is a prior action for the combined reviews. After two failed privatization attempts in 1995 and 1999, the groundnut processing company (SONACOS) was privatized in March 2005. The tax protecting SONACOS was eliminated by end-2005 as agreed. However, although this structural PC was observed in form, it has been already breached in spirit, as the authorities introduced another tax with the same protective effect in December 2005. The authorities explained that the tax conforms to the safeguard clauses of the WTO and WAEMU treaties (memorandum of economic and financial policies (MEFP, para. 14). They justified the imposition of the new tax on the basis of a surge in vegetable oil imports since 2004, which they believed had weakened SONACOS' financial position and threatened its ability to buy the groundnut crop. Staff did not find these arguments convincing and regretted the introduction of the tax - to which it had expressed strong objections - on grounds that the new tax is regressive, creates wrong incentives, and does not resolve the company's immediate financial problems.

\section{Progress was made on the poverty reduction agenda and toward achievement of} the MDGs, particularly in maternal and child mortality, and access to safe water, although not sufficient to reach the MDGs (Figure 8 and MEFP, para. 15). However, few policy initiatives targeting vulnerable groups have been introduced. The second PRSP progress report was completed at end-June 2005, and a new PRSP, building on the first PRSP and the accelerated growth strategy under elaboration in five sectors (MEFP, para. 31) is expected in early 2006.

\section{Policy Discussions}

\section{A. The Macroeconomic Outlook for 2006}

\section{The macroeconomic outlook for $\mathbf{2 0 0 6}$ is broadly favorable, although somewhat} weaker than anticipated before the recent rise in oil prices. ${ }^{4}$ Staff projections indicate that (a) real GDP growth in 2006 would be about 5 percent, as in 2005; (b) the average CPI inflation rate would rise by about one percentage point in 2006 to about 2.5 percent; and (c) the external current account deficit (including official transfers) would remain at about 7.6 percent of GDP in 2006. The main risks to the short-term economic outlook include a further sustained rise in oil prices and a slackening of political resolve to follow through with the reform agenda ahead of the elections in 2007, which could adversely affect the steady flow of external financing — on which the robust growth of the economy depends.

\footnotetext{
${ }^{4}$ The staff's projection of the average per barrel price of oil for 2005 increased from US\$37 in December 2004 to US\$54 in December 2005. Oil price movements are substantially passed through to consumers (Box 1).
} 
Figure 8. Senegal: Progress Toward Millennium Development Goals, 1990-2015 (In percent, unless otherwise indicated)
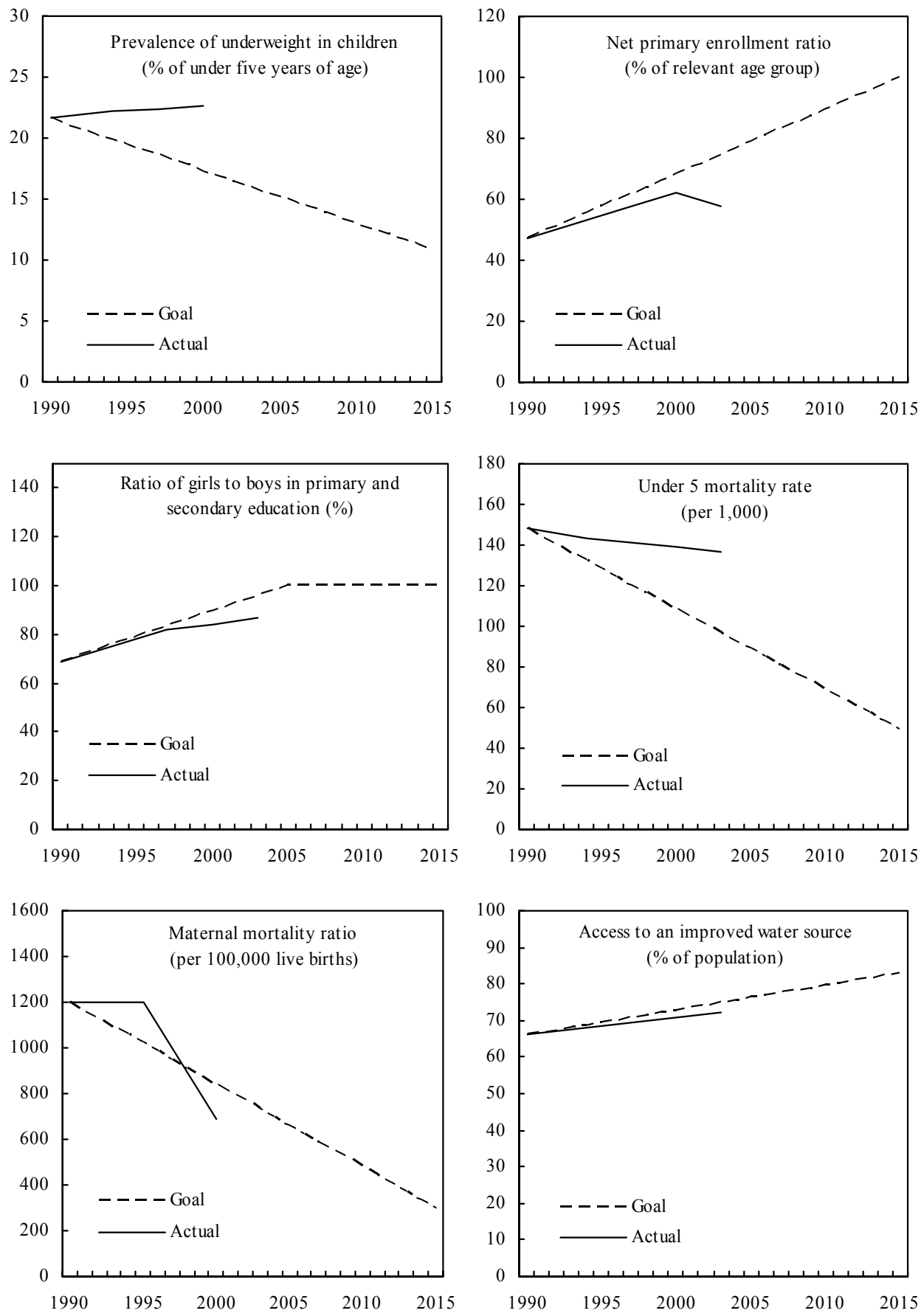

Sources: World Bank, http://www.developmentgoals.org; and United Nations, http://unstats.un.org. 


\section{B. Fiscal Policy and Reform}

16. Driven mainly by the increase in domestically financed capital expenditures, the rising trend in the overall fiscal deficit since 2000 will continue with a moderate increase in the deficit in 2006. While the 2006 budget projects an overall deficit (including grants) of 6.5 percent of GDP, the authorities agreed with staff that this level of deficit could pose a problem for macroeconomic stability. Therefore, they committed to keep the level of public investment-which has

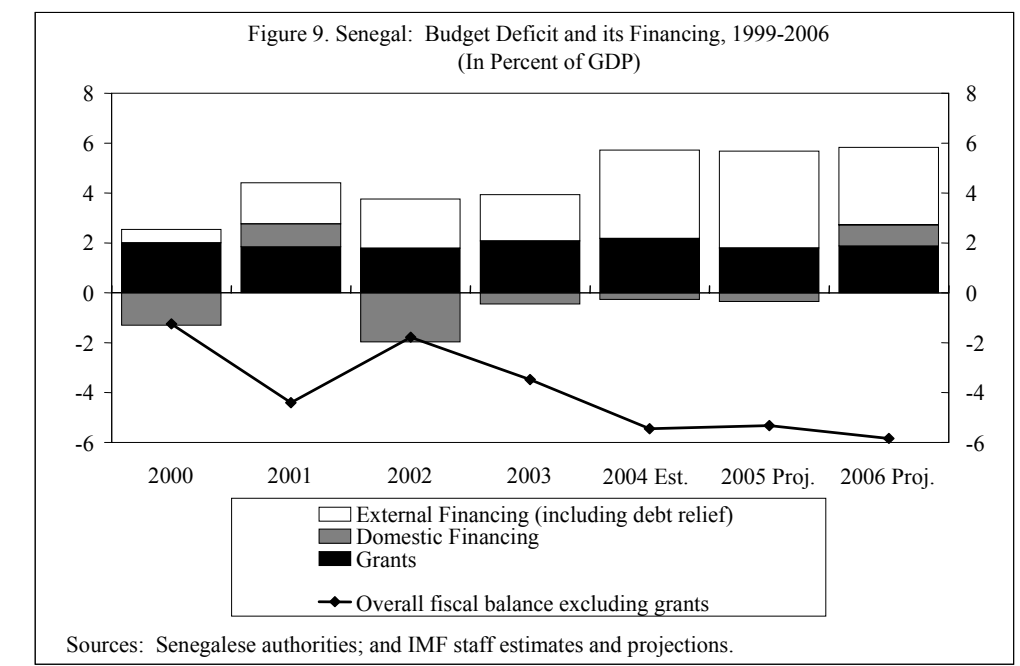
grown rapidly in recent years - below budget levels in order to contain the deficit to 4 percent of GDP. ${ }^{5}$ The basic balance would then remain positive, in line with the WAEMU convergence criterion, and there will be recourse to a moderate amount of net domestic financing in 2006, for the first time since 2001 (Figure 9). Given the low level of domestic and external public debt ( 7 percent, and 43 percent of GDP, respectively) and the concessional terms of the external debt, the composition of deficit financing, low inflation, and little likelihood of crowding out the private sector from the credit market, the fiscal outlook for 2006 is consistent with macroeconomic stability. ${ }^{6}$

\section{The authorities agreed that further increases in public investment (which has} grown from 7 percent of GDP in 2001 to more than 10 percent of GDP in 2004) should be contained, until better project planning, evaluation, and monitoring are established. ${ }^{7}$ The need for a prudent public investment program is strengthened by the insufficient productivity of these outlays (as noted by the 2005 World Bank Public Expenditure Review).

\footnotetext{
5 This adjustment was achieved by reducing domestically financed capital expenditures by about 0.5 percent of GDP and by taking absorptive capacity constraints into account for the projection of externally financed capital expenditures.

${ }^{6}$ In addition, debt service savings owing to the MDRI would average US\$90 million per year (1 percent of GDP) over the medium term.

${ }^{7}$ This includes the presentation of an economic classification of investment projects (para. 23), the regular preparation of procurement plans in the main spending ministries, and the strengthening of the medium-term expenditure framework through a better system to assess costs and benefits.
} 


\section{Box 1. Senegal-Pass-Through of Oil Price Movements}

Petroleum product prices at the pump, except for butane gas, are adjusted regularly to reflect changes in world oil prices. Domestic prices for petroleum products are set by the Conseil National des Hydrocarbures using a formula based on import prices, distribution, transportation, and retail margins, and taxes. Prices are reviewed every four weeks, and adjusted if the world oil price changes by at least 4 percent during that period. The price of butane gas is fixed and subsidized, for social and environmental policy reasons. However the subsidy is relatively large ( 0.8 percent of GDP) and poorly targeted, and will be phased out in line with WAEMU directives. The price of fuel purchased by the electricity company SENELEC from the oil refinery SAR was frozen during July-October 2005, necessitating a budgetary transfer of CFAF 3.8 billion ( 0.1 percent of GDP) to SAR.

Higher petroleum prices have had a direct impact on demand for oil imports and reduced profitability of non-oil output. In the first three quarters of 2005, crude oil import volumes were about 15 percent lower than in the corresponding period in 2004, and year-on-year growth in the industrial production index was 2 percent, compared to 10 percent in the previous year. However, the impact of the oil price increases on growth and inflation have been mitigated by the relatively low intermediate oil consumption and administered prices of electricity, butane gas, and public transport.

\section{Staff supported the significantly higher allocations for the justice and} environment sectors in the recently approved 2006 budget law, but expressed concern that the allocations for the health sector have been decreased, while those for the Presidency and the Office of the Prime Minister continue to grow. The authorities noted that some projects in the health sector had been completed and new ones will take time to develop and implement, resulting in a temporary decrease in expenditure in that sector. They also explained that the allocations for the Presidency and Prime Minister's Office had been increased because the implementation of some projects had been shifted to the agencies under their control. There was agreement that the medium-term expenditure framework had to be strengthened to prevent unwarranted decreases in priority expenditures, particularly in light of the uneven progress toward the MDGs. Staff also urged the authorities to take advantage of the savings associated with the MDRI to allocate more resources to the heath sector, other social services, and the Audit Court. The authorities noted that a supplementary budget will be prepared to account transparently for the MDRI savings and any other resources that may be received (including from the Islamic Conference Organization, the 
Millennium Challenge Account, and sale of communication licenses). The supplementary budget will allocate resources for the health and other priority sectors identified in the PRSP.

\section{Agreement was reached on procedures that would ensure transparency and} accountability in the use of the proceeds of the new air travel tax (RDIA), earmarked for Dakar new airport project. ${ }^{8}$ The authorities insisted that the proceeds of the RDIA should be kept off-budget. They did not provide a convincing justification for their preferences, but claimed that off-budget procedures would accelerate construction and facilitate financing of the project. They did not agree with staff's strong preference for the transfer of the proceeds of the RDIA to the treasury and their use according to normal budgetary procedures. Given the authorities' strong preferences, staff agreed to a second-best alternative that would ensure transparency and accountability in the use of these resources (MEFP, paras. 24-25, and Annex II). The authorities issued a decree to institute new procedures agreed with staff (a prior action for the combined review).

\section{The authorities also agreed to ensure that risks associated with the airport} project are fully accounted for. To this end (a) implementation will begin only when concerns raised by the World Bank about the project's viability are addressed, ${ }^{9}$ and the views of the Council on Infrastructure (created by law) and the Parliament are taken on board; ${ }^{10}$ (b) the contract for the construction of the new airport will be drawn up in consultation with the World Bank and Fund staffs, and submitted to the Parliament for information; (c) the contract's fiscal implications (both actual and contingent), determined in consultation with the World Bank and Fund staffs, will be reflected in the fiscal accounts; and (d) Parliamentary oversight is further assured through regular reports to the Parliament by the Minister of Finance regarding project implementation and financing (MEFP, paras. 24-25).

\section{In support of the strategy to stimulate private sector activity, the corporate} income tax was cut from 33 percent to 25 percent, effective in 2006, and a special sales tax (taxe d'égalisation) will be eliminated in 2007. Staff concurred with these policies in light of the strong revenue performance in the past two years and moderate revenue losses ( 0.3 percent of GDP) associated with these tax reforms.

\section{The mission supported recent improvements in tax administration but urged faster implementation of the long-overdue transfer of the treasury's tax collection}

\footnotetext{
${ }^{8}$ The cost of the new airport project is estimated at about 4 percent of GDP.

${ }^{9}$ These issues include (i) transparent modalities for the creation of the construction company in charge of the project, and for the selection of its shareholders; (ii) risk sharing arrangement between the government and the construction company, and the evaluation of potential contingent liabilities for the government;

(iii) management of the shift from the old to the new airport and the existing airport's assets; (iv) the size of the project and the timetable for its implementation; and (v) the control and incentive mechanisms for the management of the airport.

10 The authorities formally informed staff that parliamentary approval of the project, originally proposed by staff, was not possible under Senegal's constitution, as it impinged on the authority of the government.
} 
responsibilities to the tax department. The authorities noted important technical difficulties in the implementation of this reform, but agreed to prepare a study on its feasibility. Staff stressed that, in the meantime, the data-sharing system between the three tax collecting agencies should be made fully operational, to allow better monitoring of tax evasion and more effective assessment of the performance of each tax agency. This would require completing the long-overdue installation of the necessary software at the treasury, which the authorities committed to achieve by end-March 2006.

\section{Fiscal transparency}

23. The authorities' new action plan to address weaknesses in fiscal transparency (MEFP, Annex I) ${ }^{11}$ includes the following priority actions.

\section{- $\quad$ Procurement rules and practices will be strengthened with World Bank} assistance (MEFP, para. 9). The measures include revision of the legal and institutional frameworks in order to limit noncompetitive bids, timely preparation of procurement plans by major spending ministries, introduction of annual audits, and sanctions for infractions.

- Autonomous budget-execution agencies will be subject to strict control. A decree was issued in December 2005 to define the agencies' legal status and regulatory framework. Transfers to each agency (whether through ministerial budget allocations or directly) are clearly identified in the 2006 budget law for the first time and the practice will continue in the future. The operations of the agencies will be subject to continuous monitoring and ex post controls.

- The presentation of the table on government's financial operations (TOFE) — the main source of information on budget execution-has been revised, starting with the October 2005 table. The revision improves the transparency of fiscal outcomes (MEFP, Box 1) and brings the TOFE closer to that recommended by the WAEMU.

- $\quad$ The ability of the Audit Court to exercise control will be strengthened. Additional resources will be provided to the court in 2006 and 2007 to accelerate examination of budget review laws and the audit of treasury accounts (MEFP, Annex I). ${ }^{12}$

- Public investment expenditure will be presented according to an economic classification beginning with the 2007 budget. The presentation will facilitate the

\footnotetext{
11 The action plan is based on the recommendations of the 2003 World Bank's Country Financial Accountability Assessment (CFAA) and review of Country Procurement Practices (CPAR), the 2004 Assessment and Action Plan (AAP), and the 2005 fiscal ROSC.

12 The authorities noted that the difficulties with the new budget nomenclature and the installation of the computerized system for monitoring budget execution (SIGFIP) led to delays in transmission of these documents.
} 
measurement of recurrent costs and sharpen the distinction between capital and current expenditure.

\section{Debt management}

24. To improve domestic public debt management, the authorities are strengthening the debt unit of the Ministry of Finance. The unit will be responsible for identifying and monitoring government's existing and future domestic and external contingent liabilities arising from the activities of public enterprises. It will produce a semiannual report, the first of which will be issued in January 2006.

\section{Financial Sector Issues}

25. The banking system remains reasonably sound, but sizeable nonperforming loans (NPLs) and excessive credit concentration raise the vulnerability of the system. As of end-September 2005, NPLs had grown to 13.4 percent of total loans and provisions had declined relative to NPLs (Table 13). ${ }^{13}$ Also, loans to the five largest borrowers amounted to the equivalent of 143 percent of banks' capital. The capital adequacy ratio (CAR) for the system as a whole averaged 11.6 percent, but continued on a downward trend. The ratio for one bank slipped below the statutory 8 percent level. The mission stressed the need for a strict enforcement of prudential norms and urged the noncomplying bank be given a properly short deadline to raise its CAR.

26. As agreed during the previous review discussions, the authorities have prepared an action plan for enhancing bank soundness and improving credit availability (MEFP, Annex III). ${ }^{14}$ The priority actions include (i) simplifying the regulatory framework for loan recovery and collateral seizure to facilitate the reduction of nonperforming loans; and (ii) raising the required CAR above 8 percent, given that the structure of the economy limits the scope for reducing the risk of credit concentration. The authorities noted that they had proposed the measures that required action at the regional level to the Council of Ministers of WAEMU - its main decision-making body — for consideration. Staff also encouraged the authorities to urge speedy progress by the WAEMU on the draft law criminalizing the financing of terrorism and to update the Anti-Money Laundering law.

${ }^{13}$ As a result, the ratio of NPLs (net of provisions) to capital increased to 42 percent.

14 The action plan also reflects the recommendations of "Senegal-Financial System Stability Assessment Update," IMF Country Report No. 05/126. 


\section{Public Enterprise Reform}

27. The chemical company (ICS) - one of the largest enterprises in the country-in which the state has a 47 percent stake, is teetering on the verge of bankruptcy. ${ }^{15}$ As the largest borrower in the country (accounting for 7 percent of total bank credit), the ICS' inability to repay its loans, if not addressed, would seriously affect the soundness of some banks. The plan for its rehabilitation involves injection of capital by private shareholders. The government has agreed not to make any budgetary transfers to the company.

28. There is a need to adjust electricity prices-which were lowered by 5 percent in October 2004-to reflect the sharp increase in SENELEC's fuel costs. The government intends to raise prices gradually, with lower increases for the poor households. As a first step, prices were raised by 10 percent, on average, in November 2005. The mission urged that further price increases be implemented as needed during 2006, in light of the evolution of SENELEC's financial situation. The authorities have committed to further raising electricity prices in order to fully apply the new price formula by end-2006 (MEFP, para. 7). ${ }^{16}$

\section{E. Relations with the Fund}

29. The authorities noted their intention to continue their close collaboration with the Fund after the current arrangement expires in April 2006. They reiterated their interest in an arrangement under the Policy Support Instrument. In the meantime, in consultation with staff, the government has established a number of indicative targets to monitor the progress of its policies in 2006 (MEFP, Tables 1 and 2).

30. Senegal has an excellent record in meeting its debt service obligations to the Fund. Given the trajectory of its external and fiscal positions, it should not have any difficulty discharging its future obligations to the Fund in a timely manner (Table 12).

\section{F. Statistical Issues and Disclosure}

31. Overall, Senegal's economic database is adequate for program monitoring, but there are weaknesses in the data on national accounts, production, international trade, and social indicators. Senegal participates in the General Data Dissemination Standard (GDDS). Its metadata have been posted on the Fund's Dissemination Standards Bulletin Board since September 2001.

\footnotetext{
15 Its losses amounted to about 2 percent of GDP in 2003-04, owing mainly to overruns on investments costs, high input and low export prices during 2001-03, and the depreciation of the U.S. dollar with respect to the euro, which reduced the CFA franc value of its export earnings.

16 The 2006 fiscal program provides for sufficient transfers to SENELEC, should tariffs not be raised (Table 5).
} 


\section{STAFf APPRAisal}

32. Senegal has attained macroeconomic stability and debt sustainability, accompanied by robust economic growth, but the economy remains fragile, dependent on donor support, and vulnerable to shocks. The steady flow of external financing - on which the robust growth of the economy depends-will largely hinge on the authorities' ability to maintain a high level of credibility in the implementation of appropriate fiscal policy and structural reforms.

33. The fiscal stance for $\mathbf{2 0 0 6}$ is in line with the program's macroeconomic objectives. It allows for a modest increase in expenditure, keeps investment outlays in line with absorptive capacity of the economy and foreign financing, and aims at a realistic revenue target. However the composition of expenditure in the 2006 budget is of concern, as it shows a reduction in allocation for health services and social affairs. Staff recommends that this shift be corrected through additional allocation to health and social services in the 2006 supplementary budget.

\section{Expenditure management and fiscal transparency should be further improved.}

The decision to implement transparent procedures for the construction of the new airport is an important step in this direction. However, these procedures are second-best compared to the normal budgetary procedures; they should not be applied to other government projects. Public expenditure management should be buttressed by strict enforcement of the new procurement code in the case of ministries and autonomous agencies that execute public expenditure to reduce no-bid contracts, and by effective ex post monitoring.

35. Investment planning, evaluation, and monitoring should be improved before capital outlays are raised beyond the current levels relative to GDP. Public capital spending should remain consistent with implementation capacity and fiscal sustainability.

36. Staff welcomes the completion of the privatization of the state-owned groundnut processing company, but regrets the imposition of a new tax to protect the company. The tax is contrary to the objectives of privatization. It should be rescinded as soon as possible.

37. The prompt rehabilitation of ICS and SENELEC should be given priority. In the case of ICS, the government should press for cost cutting, management improvements, and capital injection by private partners. The financial health of SENELEC depends on its ability to adjust electricity rates in line with market conditions. To this end, and to provide incentives for investment in the electricity sector, the market-oriented formula for setting electricity prices should be adhered to.

38. Improvements in the financial sector require coordination at the regional level, but the government's action plan for the sector is a step in the right direction. Staff urges the authorities to implement the judicial reforms quickly to facilitate loan recovery. The authorities should remain vigilant in detecting fragility in the banking system, and impose timely corrective action on banks facing difficulties or in noncompliance with prudential norms. 


\section{Staff recommends the completion of the third and fourth reviews under the} PRGF arrangement. Staff also recommends waivers to be granted for the three missed performance criteria. In the short term, staff does not foresee any substantial risk on the macroeconomic front - barring supply shocks or further increases in the price of oil, and assuming the policies outlined above. The main risks stem from the possibility of policy reversals if the political will for reform slackens, especially in public finance management. However, given the government's prudent fiscal and debt policies for 2006, remedial measures already taken and planned to ensure that the program is and remains on track, and commitment to take other measures as needed, staff recommends completion of the reviews and granting of the necessary waivers. 
Table 1. Senegal: Quantitative Performance Criteria and Indicative Targets for 2005

(In billions of CFA francs, cumulative from the beginning of the year, unless otherwise specified; end of period)

\begin{tabular}{|c|c|c|c|c|c|c|c|c|c|c|c|c|c|}
\hline & \multicolumn{4}{|c|}{ March 31} & \multicolumn{4}{|c|}{ June 30} & \multicolumn{4}{|c|}{ Sept. 30} & \multirow{2}{*}{$\begin{array}{c}\text { Dec. } 31 \\
\begin{array}{c}\text { Indicative } \\
\text { Targets }\end{array}\end{array}$} \\
\hline & $\begin{array}{l}\text { Performance } \\
\text { Criteria }\end{array}$ & $\begin{array}{l}\text { Performance } \\
\text { Criteria } \\
\text { after } \\
\text { adjusters }\end{array}$ & $\begin{array}{c}\text { Actual } \\
\text { provisional }\end{array}$ & Status & $\begin{array}{l}\text { Indicative } \\
\text { Targets }\end{array}$ & $\begin{array}{l}\text { Indicative } \\
\text { Targets } \\
\text { atter } \\
\text { adjusters }\end{array}$ & $\begin{array}{c}\text { Actual } \\
\text { provisional }\end{array}$ & Status & $\begin{array}{l}\text { Performance } \\
\text { Criteria }\end{array}$ & $\begin{array}{l}\text { Performance } \\
\text { Criteria } \\
\text { atter } \\
\text { adjusters }\end{array}$ & $\begin{array}{c}\text { Actual } \\
\text { provisional }\end{array}$ & Status & \\
\hline \multicolumn{14}{|l|}{ Performance Criteria } \\
\hline \multicolumn{14}{|l|}{ Floor on the basic fiscal balance, excluding temporary costs of structural } \\
\hline Ceiling on the cumulative change in net bank credit to the government & -16.1 & 5.2 & 2.6 & met & -24.5 & -24.0 & -81.0 & met & -27.3 & -39.6 & -119.4 & met & -17.5 \\
\hline Ceiling on government domestic payments arrears $2 /$ & 0.0 & & 0.0 & met & 0.0 & & 0.0 & met & 0.0 & & 0.0 & met & 0.0 \\
\hline \multirow{2}{*}{\multicolumn{14}{|c|}{ Ceiling on the contracting or guaranteeing of new nonconcessional }} \\
\hline & & & & & & & & & & & & & \\
\hline external debt by the government $2 / 3 /$ & 0.0 & & 0.0 & met & 0.0 & & 0.0 & met & 0.0 & & 0.0 & met & 0.0 \\
\hline Ceiling on the amount of government contracts signed without budgetary allocation $2 /$ & 0.0 & & 0.0 & met & 0.0 & & 0.0 & met & 0.0 & & 0.0 & met & 0.0 \\
\hline \multirow{2}{*}{\multicolumn{14}{|c|}{ Ceiling on }} \\
\hline & & & & & & & & & & & & & \\
\hline transfers to SONACOS $2 /$ & 0.0 & & 0.0 & met & n.a. $4 /$ & & & n.a. $4 /$ & n.a. $4 /$ & & & n.a. $4 /$ & n.a. 4/ \\
\hline stock of arrears of SONACOS $2 /$ & 0.0 & & 0.0 & met & n.a. $4 /$ & & & n.a. $4 /$ & n.a. $4 /$ & & & n.a. 4/ & n.a. $4 /$ \\
\hline SONACOS' debt to the banking system & 35.5 & & $\ldots$ & n.a $4 /$ & n.a. $4 /$ & & & n.a. $4 /$ & n.a. $4 /$ & & & n.a. 4/ & n.a. $4 /$ \\
\hline \multicolumn{14}{|l|}{ Ceiling on } \\
\hline budgetary transfers to cover SENELEC operating losses $2 /$ & 0.0 & & 0.0 & met & 0.0 & & 0.0 & met & 0.0 & & 0.0 & not met $5 /$ & 0.0 \\
\hline the stock of arrears of SENELEC $2 /$ & 0.0 & & 0.9 & not met & 0.0 & & 0.9 & not met & 0.0 & & 1.0 & not met & 0.0 \\
\hline SENELEC's debt to the banking system & 52.5 & & 36.6 & met & 48.4 & & 40.3 & met & 46.6 & & 36.3 & met & 42.5 \\
\hline \multicolumn{14}{|l|}{ Indicative Targets } \\
\hline Floor on tax revenue & 176.5 & & 190.7 & met & 385.1 & & 435.6 & met & 601.6 & & 634.6 & met & 840.1 \\
\hline \multicolumn{14}{|l|}{$\begin{array}{l}\text { Ceiling on the amount of current non-wage non-interest expenditures } \\
\text { and domestically financed capital expenditures executed through }\end{array}$} \\
\hline exceptional procedures & 21.0 & & 22.0 & not met & 21.0 & & 14.0 & met & 21.0 & & 32.6 & not met & 21.0 \\
\hline Ceiling on the wage bill & 58.6 & & 61.3 & not met & 118.6 & & 124.2 & not met & 179.0 & & 185.9 & not met & 255.3 \\
\hline \multicolumn{14}{|l|}{$\begin{array}{l}\text { Floor on the difference between the net creditor flow in the treasury accounts of the postal service } \\
\text { and the net creditor flow in the deposit accounts at the Centre de Cheqques Postaux (CCP) }\end{array}$} \\
\hline and the saving accounts at the Caisse Nationale d'Epargne (CNE) $2 /$ & 0.0 & & 0.7 & met & 0.0 & & -2.0 & not met & 0.0 & & 11.9 & met & 0.0 \\
\hline \multicolumn{14}{|l|}{$\begin{array}{l}\text { Ceiling on the stock of net deposits in the correspondent accounts of the } \\
\text { treasury, excluding the correspondent accounts of local authorities, public }\end{array}$} \\
\hline agencies, SN La Poste, IPRES, and deposit and guarantee accounts & 18.0 & & 35.9 & not met & 18.0 & & 12.3 & met & 18.0 & & 29.5 & not met & 18.0 \\
\hline Ceiling on guarantee deposits of the government & 0.0 & & 0.0 & met & 0.0 & & 0.0 & met & 0.0 & & 0.0 & met & 0.0 \\
\hline \multicolumn{14}{|l|}{ Memorandum items: } \\
\hline External budgetary assistance, excluding IMF & 32.8 & & 20.1 & & 41.2 & & 25.0 & & 44.6 & & 27.3 & & 53.0 \\
\hline Grants & 3.7 & & 0.0 & & 7.7 & & 4.9 & & 11.1 & & 6.3 & & 11.5 \\
\hline Loans & 29.1 & & 20.1 & & 33.6 & & 20.1 & & 33.6 & & 21.0 & & 41.5 \\
\hline Programmed spending of HIPC debt relief & 5.9 & & 6.8 & & 27.5 & & 18.5 & & 34.3 & & 26.2 & & 69.0 \\
\hline
\end{tabular}

1/ Defined as total revenue minus total expenditure and net lending, excluding externally financed capital expenditure and on-lending, the cost of structural reforms and HIPC spending.

2/ This performance criterion or indicative target will be monitored on a continuous basis.

3/ This criterion excludes government or government-guaranteed CFAF borrowing from financial institutions within WAEMU

4/ Not applicable: SONACOS was privatized in March 2005.

$5 /$ SENELEC received budgetary transfers amounting to CFAF 16 billion in December 2005 . 
Table 2. Senegal: Prior Actions, Structural Performance Criteria, and Benchmarks for the Program under the PRGF Arrangement in 2005

\section{Measures}

\section{Proposed prior actions for the combined third and fourth reviews}

- Promulgate a decree on transparency procedures for financing and construction of Dakar's new airport.

- Improve the transparency of the Government's Table of Financial Operations (TOFE) for October 2005 in line with the methodological Changes to the government operations table explained in the box of the memorandum of economic and financial policies in 2006 .

\section{Structural performance criteria}

- Submit to the IMF staff the monthly table of the government's financial operations (TOFE) for January 2005.

- Eliminate the specific tax on refined vegetable oil.

\section{Benchmarks}

- Produce a report on direct and indirect taxes due and collected in January 2005.

- Produce reports on the execution of capital expenditures at endJune, end-September and end-December 2004. The reports will include (i) the payments authorized by the Debt and Investment Directorate for each project; (ii) the funds transferred to the accounts of these projects in the banking system by the treasury; (iii) the external funding allocated to these projects (grants, loans); and (iv) the external funds deposited in these accounts.

- Improve the transparency of the Government's Table of Financial Operations (TOFE) in line with the recommendations of the AFR/FAD technical mission of February 2004 concerning the treatment of correspondent accounts and the different government definitions used in the TOFE and the net bank credit to government.

- Prepare a report on commitments, verification, payment orders, and payment by major spending lines for the months JanuaryJune 2005, using the software "Système Intégré de Gestion des Finances Publiques” (SIGFIP).

- Implement a new formula for electricity prices compatible with operator profitability.
Implemented

Implemented

March 31, 2005

Implemented

December 31, $2005 \quad$ Implemented

March 31, 2005

Implemented with delay

March 31, 2005

Implemented

June 30, 2005

Not implemented

July 31,2005

Implemented with delay

October 1, 2005
Gradual implementation, starting in November 2005 
Table 3. Senegal: Selected Economic and Financial Indicators, 2001-06

\begin{tabular}{|c|c|c|c|c|c|c|c|c|}
\hline & \multirow[t]{2}{*}{2001} & \multirow{2}{*}{2002} & \multirow[t]{2}{*}{2003} & \multirow{2}{*}{$\begin{array}{l}2004 \\
\text { Est. }\end{array}$} & \multicolumn{2}{|c|}{2005} & \multicolumn{2}{|c|}{2006} \\
\hline & & & & & Prog. 1/ & Proj. & Proj. 1/ & Rev. proj. \\
\hline & & & Annual $\mathrm{p}$ & entage ch & ge, unless o & ise indi & & \\
\hline \multicolumn{9}{|l|}{ National income and prices } \\
\hline GDP at constant prices & 4.7 & 1.1 & 6.5 & 6.2 & 6.4 & 5.1 & 5.2 & 5.1 \\
\hline Of which : nonagriculture GDP & 4.9 & 4.9 & 4.4 & 6.3 & 6.1 & 4.7 & 5.2 & 4.8 \\
\hline GDP deflator & 0.0 & 2.7 & 0.7 & 1.9 & 2.0 & 2.6 & 2.0 & 2.3 \\
\hline \multicolumn{9}{|l|}{ Consumer prices } \\
\hline Annual average & 3.0 & 2.3 & 0.0 & 0.5 & 1.6 & 1.8 & 1.9 & 2.6 \\
\hline End of period & 3.9 & 1.5 & -1.4 & 1.7 & 1.6 & 2.1 & 1.9 & 2.2 \\
\hline \multicolumn{9}{|l|}{ External sector } \\
\hline Exports, f.o.b. (in CFA francs) & 12.3 & 1.1 & -1.7 & 6.1 & 6.2 & 8.6 & 5.5 & 6.5 \\
\hline Imports, f.o.b. (in CFA francs) & 10.0 & 6.8 & 7.4 & 9.0 & 2.3 & 9.5 & 5.5 & 6.7 \\
\hline Export volume & 8.7 & 3.2 & 0.1 & 3.6 & 4.3 & 3.1 & 4.3 & 4.1 \\
\hline Import volume & 12.5 & 6.6 & 4.1 & 5.9 & 5.4 & 1.9 & 4.6 & 3.4 \\
\hline Terms of trade (deterioration -) & 5.7 & -1.0 & -5.5 & -0.6 & 4.1 & -1.9 & 0.7 & -0.5 \\
\hline Nominal effective exchange rate $2 /$ & 1.2 & 2.4 & 5.2 & 1.9 & $\ldots$ & -0.4 & $\ldots$ & $\cdots$ \\
\hline \multirow[t]{2}{*}{ Real effective exchange rate $2 /$} & 1.8 & 2.8 & 2.7 & 0.1 & $\ldots$ & -1.9 & $\ldots$ & $\cdots$ \\
\hline & \multicolumn{8}{|c|}{ (Changes in percent of beginning-of-year broad money, unless otherwise indicated) } \\
\hline \multicolumn{9}{|l|}{ Money and credit } \\
\hline Net domestic assets & 4.3 & -6.4 & 5.4 & 3.1 & 3.8 & 11.7 & 4.2 & 8.3 \\
\hline Domestic credit & 6.6 & -4.9 & 5.7 & 2.5 & 4.2 & 11.5 & 4.4 & 8.4 \\
\hline Credit to the government (net) & 2.7 & -8.3 & -4.3 & -3.1 & 0.2 & -1.2 & -0.9 & 2.4 \\
\hline Credit to the economy (percentage growth) & 4.9 & 4.7 & 14.3 & 9.2 & 5.7 & 21.5 & 7.7 & 9.5 \\
\hline Broad money (M2) & 14.5 & 7.6 & 31.5 & 12.9 & 8.5 & 16.0 & 6.3 & 11.0 \\
\hline Velocity (GDP/M2; end of period) & 3.7 & 3.6 & 2.9 & 2.8 & 3.4 & 2.6 & 3.4 & 2.5 \\
\hline \multicolumn{9}{|l|}{ Interest rates (end of period; in percent) } \\
\hline Discount rate & 6.50 & 5.00 & 5.00 & 4.50 & $\ldots$ & $\ldots$ & $\ldots$ & ... \\
\hline \multirow[t]{2}{*}{ Money market rate } & 4.95 & 4.95 & 4.95 & 4.95 & $\cdots$ & $\cdots$ & $\cdots$ & $\cdots$ \\
\hline & \multicolumn{8}{|c|}{ (In percent of GDP) } \\
\hline \multicolumn{9}{|l|}{ Government financial operations } \\
\hline Revenue & 18.0 & 19.1 & 19.3 & 19.3 & 19.3 & 20.2 & 19.3 & 20.0 \\
\hline Grants & 1.8 & 1.8 & 2.1 & 2.2 & 1.9 & 1.8 & 2.2 & 1.9 \\
\hline Total expenditure and net lending & 22.3 & 21.6 & 23.1 & 24.5 & 24.1 & 25.6 & 22.9 & 25.8 \\
\hline \multicolumn{9}{|l|}{ Overall fiscal surplus or deficit (-) } \\
\hline Payment order basis, excluding grants & -4.4 & -1.8 & -3.5 & -5.5 & -4.8 & -5.3 & -3.5 & -5.8 \\
\hline Payment order basis, including grants & -2.6 & 0.0 & -1.4 & -3.3 & -2.9 & -3.5 & -1.4 & -4.0 \\
\hline Primary fiscal balance $3 /$ & -1.5 & 0.5 & -0.5 & -1.9 & -1.9 & -2.5 & -0.4 & -3.0 \\
\hline Basic fiscal balance, program definition 4/ & -0.8 & 2.1 & 1.3 & 0.8 & 0.7 & 0.9 & 0.8 & 0.0 \\
\hline Gross domestic investment & 19.2 & 16.7 & 20.7 & 23.4 & 23.4 & 23.4 & 22.6 & 23.7 \\
\hline Gross domestic savings & 9.5 & 5.6 & 7.7 & 9.8 & 12.1 & 9.3 & 11.6 & 9.8 \\
\hline Gross national savings & 14.5 & 10.8 & 14.1 & 16.7 & 17.9 & 15.7 & 17.5 & 16.1 \\
\hline \multicolumn{9}{|l|}{ External current account deficit (-) } \\
\hline Excluding current official transfers & -6.3 & -8.0 & -8.6 & -8.7 & -7.1 & -9.2 & -6.9 & -9.1 \\
\hline Including current official transfers & -4.7 & -6.0 & -6.6 & -6.7 & -5.4 & -7.6 & -5.1 & -7.6 \\
\hline Domestic public debt $6 /$ & 9.2 & 7.2 & 6.8 & 6.5 & 6.5 & 7.0 & 5.6 & 6.6 \\
\hline External public debt (nominal) $5 / 6 /$ & 67.3 & 66.1 & 54.5 & 46.3 & 38.3 & 42.8 & 35.4 & 39.9 \\
\hline NPV of external debt 5/ 7/ & $\ldots$ & $\ldots$ & $\ldots$ & 33.7 & 28.2 & 32.2 & 27.1 & 31.9 \\
\hline NPV of external debt (in percent of exports) 5/7/ & $\ldots$ & $\ldots$ & $\ldots$ & 121.2 & 104.7 & 116.4 & 104.8 & 117.1 \\
\hline & & percent & exports c & oods and & ffactor serv & unless $\mathrm{C}$ & rwise indic & \\
\hline External public debt service 5/ & 9.0 & 9.2 & 8.3 & 6.9 & 6.4 & 6.0 & 7.1 & 6.9 \\
\hline In percent of government revenue & 15.3 & 14.8 & 12.3 & 9.9 & 9.0 & 8.2 & 9.6 & 9.4 \\
\hline GDP at current market prices (in billions of CFA francs) & 3,343 & 3,473 & 3,725 & 4,029 & 4,364 & 4,346 & 4,682 & 4,673 \\
\hline
\end{tabular}

Sources: Senegalese authorities; and staff estimates and projections.

1/ Staff Report for the 2004 Article IV consultation and the second review.

2/ First 9 months in 2005 .

3/ Defined as total revenue and grants minus total expenditure and net lending, excluding interest expenditure.

4/ Defined as total revenue minus total expenditure and net lending, excluding externally financed capital expenditure, on-lending,

temporary costs of structural reforms and expenditure financed with HIPC Initiative assistance.

5/ After HIPC debt relief.

6/ Debt outstanding at year-end.

7/ Including programmed new debt. 
Table 4. Senegal: Savings-Investment Balances and National Accounts 2001-06

\begin{tabular}{|c|c|c|c|c|c|c|c|c|c|}
\hline & \multirow{2}{*}{$\begin{array}{l}\text { Composition } \\
\text { of GDP in } 1999 \\
\text { (In percent) }\end{array}$} & \multirow{2}{*}{2001} & \multirow{2}{*}{2002} & \multirow{2}{*}{2003} & \multirow{2}{*}{$\begin{array}{l}2004 \\
\text { Est. }\end{array}$} & \multicolumn{2}{|c|}{2005} & \multicolumn{2}{|c|}{2006} \\
\hline & & & & & & Prog. 1/ & Proj. & Proj. 1/ & Rev. proj. \\
\hline & \multicolumn{9}{|c|}{ (Annual percentage change at constant prices, unless otherwise indicated) } \\
\hline Primary sector & 18.1 & 3.3 & -20.6 & 19.8 & 4.3 & 7.3 & 7.4 & 3.8 & 6.0 \\
\hline Agriculture & 9.9 & 3.0 & -32.2 & 36.3 & 4.2 & 9.5 & 10.0 & 5.3 & 8.0 \\
\hline Livestock & 5.1 & 4.5 & -6.4 & 4.2 & 5.0 & 5.0 & 5.0 & 4.0 & 4.0 \\
\hline Forestry & 0.8 & 3.0 & 2.3 & 4.0 & 4.0 & 3.0 & 3.2 & 3.0 & 3.0 \\
\hline Fishing & 2.4 & 2.3 & -6.4 & 4.9 & 2.4 & 4.0 & 1.9 & -5.0 & 1.9 \\
\hline Secondary sector & 18.7 & 4.3 & 9.8 & 6.4 & 5.8 & 6.2 & 3.1 & 6.5 & 3.6 \\
\hline Mining & 1.3 & 0.7 & 11.3 & 28.0 & -11.9 & 4.3 & -13.0 & 4.0 & 3.5 \\
\hline Industry & 11.6 & 6.1 & 11.5 & 1.4 & 7.0 & 4.3 & 1.6 & 5.0 & 0.1 \\
\hline Oil milling & 0.2 & 6.2 & -9.6 & -22.8 & -17.9 & 10.0 & 26.8 & 10.0 & 15.0 \\
\hline Energy & 1.9 & 6.7 & -2.7 & 18.4 & 1.1 & 5.0 & 0.9 & 5.7 & 1.5 \\
\hline Construction and public works & 3.6 & -1.0 & 12.4 & 10.5 & 14.0 & 13.0 & 13.0 & 11.6 & 13.0 \\
\hline Tertiary sector & 63.2 & 5.2 & 4.7 & 3.7 & 6.7 & 6.2 & 5.3 & 5.1 & 5.4 \\
\hline Transportation and telecommunications & 6.3 & 14.1 & 5.5 & 7.3 & 13.3 & 9.0 & 10.4 & 7.5 & 9.0 \\
\hline Commerce & 18.5 & 1.7 & 5.9 & 4.2 & 6.3 & 6.7 & 4.6 & 4.0 & 4.0 \\
\hline Public administration & 19.7 & 7.5 & 3.1 & 2.2 & 4.7 & 5.4 & 5.4 & 4.8 & 5.4 \\
\hline Other & 18.7 & 2.9 & 5.1 & 3.5 & 6.7 & 5.3 & 3.4 & 5.3 & 4.9 \\
\hline GDP & 100.0 & 4.7 & 1.1 & 6.5 & 6.2 & 6.4 & 5.1 & 5.2 & 5.1 \\
\hline Nonagriculture GDP & 90.1 & 4.9 & 4.9 & 4.4 & 6.3 & 6.1 & 4.7 & 5.2 & 4.8 \\
\hline GDP deflator & $\ldots$ & 0.0 & 2.7 & 0.7 & 1.9 & 2.0 & 2.6 & 2.0 & 2.3 \\
\hline \multirow[t]{2}{*}{ Consumer price index (period average) } & & 3.0 & 2.3 & 0.0 & 0.5 & 1.6 & 1.8 & 1.9 & 2.6 \\
\hline & \multicolumn{9}{|c|}{ (In percent of GDP) } \\
\hline Gross domestic investment & & 19.2 & 16.7 & 20.7 & 23.4 & 23.4 & 23.4 & 22.6 & 23.7 \\
\hline Government 2/ & & 6.9 & 7.9 & 9.1 & 10.2 & 9.7 & 9.8 & 8.9 & 10.1 \\
\hline Nongovernment & & 12.2 & 8.8 & 11.6 & 13.2 & 13.7 & 13.6 & 13.7 & 13.6 \\
\hline Gross domestic savings & & 9.5 & 5.6 & 7.7 & 9.8 & 12.1 & 9.3 & 11.6 & 9.8 \\
\hline Government & & 5.4 & 9.0 & 8.9 & 8.8 & 10.0 & 6.5 & 9.8 & 6.6 \\
\hline Nongovernment & & 4.2 & -3.4 & -1.1 & 0.9 & 2.1 & 2.8 & 1.8 & 3.3 \\
\hline Savings - investment balance & & -9.7 & -11.2 & -13.0 & -13.6 & -11.2 & -14.0 & -11.0 & -13.8 \\
\hline Government & & -1.6 & 1.0 & -0.2 & -1.3 & 0.3 & -3.3 & 0.9 & -3.5 \\
\hline Nongovernment & & -8.1 & -12.2 & -12.8 & -12.3 & -11.5 & -10.7 & -11.9 & -10.3 \\
\hline External current account balance 3/ & & -4.7 & -6.0 & -6.6 & -6.7 & -5.4 & -7.6 & -5.1 & -7.6 \\
\hline Gross national savings & & 14.5 & 10.8 & 14.1 & 16.7 & 17.9 & 15.7 & 17.5 & 16.1 \\
\hline Memorandum item: & & & & (In bil & ns of CF & francs) & & & \\
\hline GDP at current prices & & 3,343 & 3,473 & 3,725 & 4,029 & 4,364 & 4,346 & 4,682 & 4,673 \\
\hline
\end{tabular}

Sources: Senegalese authorities; and staff estimates and projections.

1/ Staff Report for the 2004 Article IV consultation and the second review.

2/ Includes capital expenditure financed with HIPC Initiative assistance.

3/ Includes current official transfers. 
Table 5. Senegal: Government Financial Operations, 2001-06

\begin{tabular}{|c|c|c|c|c|c|c|c|c|c|c|c|c|}
\hline & \multicolumn{2}{|c|}{2001} & \multicolumn{2}{|c|}{2002} & \multicolumn{2}{|c|}{2003} & \multicolumn{2}{|c|}{2004} & \multicolumn{2}{|c|}{2005} & \multicolumn{2}{|c|}{2006} \\
\hline & Old $1 /$ & $\overline{\text { New } 2 /}$ & Old $1 /$ & New $2 /$ & Old $1 /$ & $\overline{\text { New } 2 /}$ & Old est. 1/ & $\overline{\text { New est. } 2 /}$ & Prog. 1/3/ & Proj. $2 /$ & Proj. 1/3/ & Rev. Proj. $2 /$ \\
\hline & \multicolumn{12}{|c|}{ (In billions of CFA francs) } \\
\hline Total revenue and grants & 664.4 & 664.4 & 726.7 & 726.7 & 797.8 & 797.8 & 864.8 & 864.8 & 922.4 & 957.0 & $1,006.1$ & $1,022.4$ \\
\hline Revenue & 602.7 & 602.7 & 664.6 & 664.6 & 720.1 & 720.1 & 776.8 & 776.8 & 841.0 & 879.0 & 905.1 & 934.5 \\
\hline Tax revenue & 576.8 & 576.8 & 629.2 & 629.2 & 677.0 & 677.0 & 738.5 & 738.5 & 802.1 & 840.1 & 862.7 & 892.1 \\
\hline Nontax revenue & 25.9 & 25.9 & 35.4 & 35.4 & 43.1 & 43.1 & 38.3 & 38.3 & 38.9 & 38.9 & 42.4 & 42.4 \\
\hline Grants & 61.7 & 61.7 & 62.1 & 62.1 & 77.7 & 77.7 & 88.0 & 88.0 & 81.4 & 78.1 & 101.0 & 87.9 \\
\hline $\begin{array}{l}\text { Budgetary } \\
\text { Budgeted develonment proiects }\end{array}$ & 0.0 & 0.0 & 1.9 & 1.9 & 17.6 & 17.6 & 18.5 & 18.5 & 14.8 & 11.5 & 29.1 & 16.0 \\
\hline Budgeted development projects & 61.7 & 61.7 & 60.2 & 60.2 & 60.1 & 60.1 & 69.5 & 69.5 & 66.6 & 66.6 & 71.9 & 71.9 \\
\hline Total expenditure and net lending & 748.1 & 744.3 & 730.3 & 748.5 & 850.1 & 861.2 & 962.7 & 987.8 & $1,049.8$ & $1,110.4$ & $1,071.2$ & $1,207.3$ \\
\hline Current expenditure & 516.6 & 516.6 & 478.2 & 478.2 & 529.5 & 529.5 & 553.9 & 553.9 & 600.4 & 658.2 & 647.5 & 700.7 \\
\hline Wages and salaries & 177.3 & 177.3 & 199.4 & 199.4 & 203.7 & 203.7 & 217.6 & 217.6 & 247.3 & 255.3 & 267.1 & 266.3 \\
\hline Interest due & 30.3 & 30.3 & 39.8 & 39.8 & 44.6 & 44.6 & 46.7 & 46.7 & 43.2 & 43.7 & 47.0 & 46.3 \\
\hline Of which: external & 23.7 & 23.7 & 35.4 & 35.4 & 40.0 & 40.0 & 41.1 & 41.1 & 37.9 & 38.3 & 36.0 & 37.9 \\
\hline Other current expenditure & 309.0 & 309.0 & 239.0 & 239.0 & 281.2 & 281.2 & 289.6 & 289.6 & 309.9 & 359.2 & 333.5 & 388.1 \\
\hline Transfers and subsidies & 178.5 & 178.5 & ... & & 140.9 & 140.9 & 135.4 & 135.4 & 142.9 & 193.9 & 152.9 & 198.3 \\
\hline $\begin{array}{l}\text { Of which : SAR } \\
\text { Of which SENELEC }\end{array}$ & 0.0 & 0.0 & 0.0 & 0.0 & 0.0 & 0.0 & 9.2 & 9.2 & 9.2 & 43.0 & 9.2 & 35.0 \\
\hline $\begin{array}{l}\text { Of which : SENELEC } \\
\text { Goods and services }\end{array}$ & $\begin{array}{r}40.0 \\
130.5\end{array}$ & $\begin{array}{r}0.0 \\
1305\end{array}$ & 0.0 & 0.0 & 0.0 & 0.0 & $\begin{array}{r}0.0 \\
1480\end{array}$ & $\begin{array}{r}0.0 \\
1480\end{array}$ & $\begin{array}{r}0.0 \\
1570\end{array}$ & ${ }_{155.0}^{16.0}$ & $\begin{array}{r}0.0 \\
1806\end{array}$ & 28.1 \\
\hline $\begin{array}{l}\text { Goods and services } \\
\text { HIPC current spending }\end{array}$ & 130.5 & 130.5 & $\cdots$ & $\cdots$ & 140.3 & 140.3 & $\begin{aligned} 148.0 \\
62\end{aligned}$ & $\begin{aligned} 148.0 \\
62\end{aligned}$ & $\begin{array}{l}157.0 \\
10.0\end{array}$ & $\begin{array}{l}155.3 \\
100\end{array}$ & $\begin{aligned} 180.6 \\
0.0\end{aligned}$ & $\begin{array}{l}181.1 \\
86\end{array}$ \\
\hline Capital expenditure & 232.3 & 232.3 & 275.9 & 275.9 & 338.5 & 338.5 & $\begin{array}{r}6.2 \\
410.3\end{array}$ & $\begin{array}{r}6.2 \\
410.3\end{array}$ & $\begin{array}{r}102.0 \\
42.9\end{array}$ & $\begin{array}{r}10.0 \\
425.2\end{array}$ & $\begin{array}{r}0.0 \\
415.7\end{array}$ & $\begin{array}{r}8.6 \\
473.1\end{array}$ \\
\hline Domestically financed & 133.6 & 133.6 & 147.9 & 147.9 & 190.3 & 190.3 & 221.3 & 221.3 & 279.0 & 257.4 & 259.0 & 295.0 \\
\hline HIPC financed & 15.1 & 15.1 & 4.1 & 4.1 & 28.4 & 28.4 & 22.5 & 22.5 & 49.6 & 59.0 & 30.5 & 44.5 \\
\hline Non HIPC financed & 118.5 & 118.5 & 143.8 & 143.8 & 161.9 & 161.9 & 198.8 & 198.8 & 229.4 & 198.4 & 228.6 & 250.5 \\
\hline Externally financed & 98.7 & 98.7 & 128.0 & 128.0 & 148.2 & 148.2 & 189.0 & 189.0 & 143.9 & 167.8 & 156.7 & 178.1 \\
\hline Treasury special accounts and correspondents (net) & 3.8 & & -18.2 & & -11.1 & & -25.1 & & 0.0 & & 0.0 & \\
\hline Net lending & -4.6 & -4.6 & -5.6 & -5.6 & -6.8 & -6.8 & 12.3 & 12.3 & 8.0 & 23.0 & 8.0 & 18.0 \\
\hline Temporary costs of structural reforms & 0.0 & 0.0 & 0.0 & 0.0 & 0.0 & 0.0 & 11.3 & 11.3 & 18.5 & 4.0 & 0.0 & 15.5 \\
\hline Selected public sector entities balance 4 / & $\ldots$ & -5.8 & $\ldots$ & 21.9 & $\ldots$ & 11.5 & $\ldots$ & -8.7 & $\ldots$ & 0.0 & $\ldots$ & 0.0 \\
\hline Primary fiscal balance 5 / & -53.4 & -55.4 & 36.2 & 39.9 & -7.7 & -7.4 & 11.3 & -85.0 & -84.2 & -109.7 & -18.1 & -138.6 \\
\hline $\begin{array}{l}\text { Overall fiscal balance (including grants) } \\
\text { Overall fiscal balance (excluding grants) }\end{array}$ & $\begin{array}{r}-83.7 \\
-145.4\end{array}$ & $\begin{array}{r}-85.7 \\
-147.4\end{array}$ & $\begin{array}{r}-3.6 \\
-65.7\end{array}$ & $\begin{array}{r}0.1 \\
-62.0\end{array}$ & $\begin{array}{r}-52.3 \\
-130.0\end{array}$ & $\begin{array}{r}-52.0 \\
-129.7\end{array}$ & $\begin{array}{r}-97.9 \\
-185.9\end{array}$ & $\begin{array}{l}-131.7 \\
-219.7\end{array}$ & $\begin{array}{l}-127.5 \\
-208.8\end{array}$ & $\begin{array}{l}-153.3 \\
-231.4\end{array}$ & $\begin{array}{r}-65.1 \\
-166.1\end{array}$ & $\begin{array}{l}-184.9 \\
-272.8\end{array}$ \\
\hline Basic fiscal balance (program definition) 6/ & -25.9 & -27.9 & 69.1 & 72.8 & 49.3 & 49.6 & 67.1 & 33.3 & 29.2 & 40.4 & 37.1 & 0.0 \\
\hline Financing & 83.7 & 85.7 & 3.6 & -0.1 & 52.3 & 52.0 & 97.9 & 131.7 & 127.5 & 153.3 & 65.1 & 184.9 \\
\hline External financing & 54.9 & 54.9 & 68.4 & 68.4 & 68.9 & 68.9 & 142.3 & 142.3 & 139.2 & 168 & 78.0 & 145.5 \\
\hline Drawings & 103.3 & 103.3 & 112.7 & 112.7 & 90.8 & 90.8 & 156.6 & 156.6 & 148.6 & 173.7 & 127.8 & 164.1 \\
\hline Program loans & 60.6 & 60.6 & 42.2 & 42.2 & 0.0 & 0.0 & 13.1 & 13.1 & 55.3 & 41.5 & 27.0 & 31.9 \\
\hline Project loans & 42.7 & 42.7 & 70.5 & 70.5 & 90.8 & 90.8 & 143.5 & 143.5 & 93.3 & 132.2 & 100.8 & 132.2 \\
\hline Amortization due & -64.2 & -64.2 & -81.0 & -81.0 & -73.9 & -73.9 & -126.9 & -126.9 & -127.1 & -129.7 & -128.4 & -132.0 \\
\hline Debt relief and HIPC Initiative assistance $7 /$ & 15.8 & 15.8 & 36.7 & 36.7 & 44.0 & 44.0 & 109.2 & 109.2 & 105.7 & 112.4 & 93.1 & 98.4 \\
\hline T-bills and bonds issued in WAEMU & 0.0 & 0.0 & 0.0 & 0.0 & 8.0 & 8.0 & 3.4 & 3.4 & 12.1 & 12.1 & -14.4 & 15.0 \\
\hline Domestic financing & 17.4 & 10.8 & -70.7 & -74.4 & -16.5 & -11.6 & -42.3 & -8.4 & -11.8 & -15.2 & -13.0 & 39.3 \\
\hline Banking system & 21.8 & 39.9 & -75.4 & -67.0 & -42.3 & -13.9 & -40.2 & -49.2 & 2.5 & -17 & -11.3 & 41.0 \\
\hline Of which: T-bills and bonds & & & & & 15.0 & 15.0 & 18.9 & 18.9 & 36.6 & 36.6 & 2.8 & 15.0 \\
\hline Nonbank financing & -4.4 & -29.1 & 4.7 & -7.4 & 25.8 & 2.2 & -2.1 & 40.8 & -14.3 & 2.3 & -1.7 & -1.7 \\
\hline $\begin{array}{l}\text { Of which : privatization } \\
\text { correspondents' accounts }\end{array}$ & -44.1 & $\begin{array}{r}-44.1 \\
2.0\end{array}$ & 1.1 & $\begin{array}{r}1.1 \\
-3.7\end{array}$ & 1.1 & $\begin{array}{r}1.1 \\
-0.4\end{array}$ & 1.1 & $\begin{array}{r}1.1 \\
33.8\end{array}$ & 1.1 & 6.4 & 1.1 & 1.1 \\
\hline Errors and omissions & 114 & 200 & 59 & 59 & & -04 & -21 & -22 & 00 & 00 & 00 & 00 \\
\hline $\begin{array}{l}\text { Errors and omissions } \\
\text { Financing gap }\end{array}$ & 0.0 & 0.0 & 0.0 & 0.0 & 0.0 & 0.0 & 0.0 & 0.0 & 0.0 & 0.0 & 0.0 & 0.0 \\
\hline Memor & & & & & & & & & & & & \\
\hline Airport travel tax earmarked for new airport (RDIA) & & & & & & & & & & 11.5 & & 16.1 \\
\hline HIPC Initiative expenditure 8/ & 15.1 & 15.1 & 4.1 & 4.1 & 28.4 & 28.4 & 28.7 & 28.7 & 59.6 & 69.0 & 30.5 & 53.2 \\
\hline $\begin{array}{l}\text { Banking deposits without counterpart in the fiscal accounts } \\
\text { Gross domestic product }\end{array}$ & $3,342.7$ & $\begin{array}{r}-18.1 \\
3.342 .7\end{array}$ & $3,472.7$ & $\begin{array}{r}-8.4 \\
3.772 .7\end{array}$ & $3,725.4$ & $3 .-28.4$ & 40292 & $\begin{array}{r}9.0 \\
4.029 .2\end{array}$ & $4,364.3$ & 4345.5 & $4,682.1$ & 4.6727 \\
\hline
\end{tabular}

Sources: Senegalese authorities; and staff estimates and projections.

1/ Before methodological changes presented in the box of the Memorandum of Economic and Financial Policies.

2/ Incorporates methodological changes presented in the box 1 the Memorandum of Economic and Financial Policies.

$3 /$ Staff Report for the 2004 Article IV consultation and the second review.

4/ Local governments, autonomous public sector entities (e.g. hospitals, universities), and the civil servants' pension fund (FNR).

6/ Defined as total revenue minus total expenditure and net lending, excluding externally financed capital expenditure, on-lending, cost of structural reforms and HIPC expenditure.

8/ Refers to HIPC-financed current spending in 2001, and, for 2002-06, HIPC-financed capital and other expenditure authorized in the supplement budgets of 2001, 2003 and 2004, and expenditures expected in 2005-06. 
Table 6. Senegal: Government Financial Operations, 2001-06

\begin{tabular}{|c|c|c|c|c|c|c|c|c|c|c|c|c|}
\hline & \multicolumn{2}{|c|}{2001} & \multicolumn{2}{|c|}{2002} & \multicolumn{2}{|c|}{2003} & \multicolumn{2}{|c|}{2004} & \multicolumn{2}{|c|}{2005} & \multicolumn{2}{|c|}{2006} \\
\hline & Old $1 /$ & $\mathrm{New} 2 /$ & Old $1 /$ & New $2 /$ & Old $1 /$ & $\overline{\text { New } 2 /}$ & Old est. 1/ & $\overline{\text { New est. } 2 /}$ & Prog. $1 / 3 /$ & $\overline{\text { Proj. } 21}$ & Proj. 1/3/ & $\overline{\text { Rev. Proj. } 2 /}$ \\
\hline & \multicolumn{12}{|c|}{ In percent of GDP } \\
\hline Total revenue and grants & 19.9 & 19.9 & 20.9 & 20.9 & 21.4 & 21.4 & 21.5 & 21.5 & 21.1 & 22.0 & 21.5 & 21.9 \\
\hline Revenue & 18.0 & 18.0 & 19.1 & 19.1 & 19.3 & 19.3 & 19.3 & 19.3 & 19.3 & 20.2 & 19.3 & 20.0 \\
\hline Tax revenue & 17.3 & 17.3 & 18.1 & 18.1 & 18.2 & 18.2 & 18.3 & 18.3 & 18.4 & 19.3 & 18.4 & 19.1 \\
\hline Nontax revenue & 0.8 & 0.8 & 1.0 & 1.0 & 1.2 & 1.2 & 1.0 & 1.0 & 0.9 & 0.9 & 0.9 & 0.9 \\
\hline Grants & 1.8 & 1.8 & 1.8 & 1.8 & 2.1 & 2.1 & 2.2 & 2.2 & 1.9 & 1.8 & 2.2 & 1.9 \\
\hline Total expenditure and net lending & 22.4 & 22.3 & 21.0 & 21.6 & 22.8 & 23.1 & 23.9 & 24.5 & 24.1 & 25.6 & 22.9 & 25.8 \\
\hline Current expenditure & 15.5 & 15.5 & 13.8 & 13.8 & 14.2 & 14.2 & 13.7 & 13.7 & 13.8 & 15.1 & 13.8 & 15.0 \\
\hline Wages and salaries & 5.3 & 5.3 & 5.7 & 5.7 & 5.5 & 5.5 & 5.4 & 5.4 & 5.7 & 5.9 & 5.7 & 5.7 \\
\hline Interest payments & 0.9 & 0.9 & 1.1 & 1.1 & 1.2 & 1.2 & 1.2 & 1.2 & 1.0 & 1.0 & 1.0 & 1.0 \\
\hline Other current expenditure & 9.2 & 9.2 & 6.9 & 6.9 & 7.5 & 7.5 & 7.2 & 7.2 & 7.1 & 8.3 & 7.1 & 8.3 \\
\hline Goods and services & 3.9 & 3.9 & $\ldots$ & $\ldots$ & 3.8 & 3.8 & 3.7 & 3.7 & 3.6 & 3.6 & 3.9 & 3.9 \\
\hline Transfers and subsidies & 5.3 & 5.3 & $\ldots$ & $\ldots$ & 3.8 & 3.8 & 3.4 & 3.4 & 3.3 & 4.5 & 3.3 & 4.2 \\
\hline Capital expenditure & 6.9 & 6.9 & 7.9 & 7.9 & 9.1 & 9.1 & 10.2 & 10.2 & 9.7 & 9.8 & 8.9 & 10.1 \\
\hline Domestically financed & 4.0 & 4.0 & 4.3 & 4.3 & 5.1 & 5.1 & 5.5 & 5.5 & 6.4 & 5.9 & 5.5 & 6.3 \\
\hline Externally financed & 3.0 & 3.0 & 3.7 & 3.7 & 4.0 & 4.0 & 4.7 & 4.7 & 3.3 & 3.9 & 3.3 & 3.8 \\
\hline Treasury special accounts and correspondents (net) & 0.1 & $\ldots$ & -0.5 & & -0.3 & $\ldots$ & -0.6 & $\ldots$ & 0.0 & $\ldots$ & 0.0 & $\ldots$ \\
\hline Net lending & -0.1 & -0.1 & -0.2 & -0.2 & -0.2 & -0.2 & 0.3 & 0.3 & 0.2 & 0.5 & 0.2 & 0.4 \\
\hline Temporary costs of structural reforms & 0.0 & 0.0 & 0.0 & 0.0 & 0.0 & 0.0 & 0.3 & 0.3 & 0.4 & 0.1 & 0.0 & 0.3 \\
\hline Selected public sector entities balance 4 / & $\ldots$ & -0.2 & $\ldots$ & 0.6 & $\ldots$ & 0.3 & $\ldots$ & -0.2 & $\ldots$ & 0.0 & $\ldots$ & 0.0 \\
\hline Primary fiscal balance 5/ & -1.6 & -1.5 & 1.0 & 0.5 & -0.2 & -0.5 & -1.3 & -1.9 & -1.9 & -2.5 & -0.4 & -3.0 \\
\hline \multicolumn{13}{|l|}{ Overall fiscal balance } \\
\hline Payment order basis, excluding grants & -4.3 & -4.4 & -1.9 & -1.8 & -3.5 & -3.5 & -4.6 & -5.5 & -4.8 & -5.3 & -3.5 & -5.8 \\
\hline Payment order basis, including grants & -2.5 & -2.6 & -0.1 & 0.0 & -1.4 & -1.4 & -2.4 & -3.3 & -2.9 & -3.5 & -1.4 & -4.0 \\
\hline Basic fiscal balance (program definition ) $6 /$ & -0.8 & -0.8 & 2.0 & 2.1 & 1.3 & 1.3 & 1.7 & 0.8 & 0.7 & 0.9 & 0.8 & 0.0 \\
\hline Financing & 2.5 & 2.6 & 0.1 & 0.0 & 1.4 & 1.4 & 2.4 & 3.3 & 2.9 & 3.5 & 1.4 & 4.0 \\
\hline External financing & 1.6 & 1.6 & 2.0 & 2.0 & 1.8 & 1.8 & 3.5 & 3.5 & 2.9 & 3.9 & 2.0 & 3.1 \\
\hline Domestic financing & 0.5 & 0.3 & -2.0 & -2.1 & -0.4 & -0.3 & -1.0 & -0.2 & 0.0 & -0.3 & -0.6 & 0.8 \\
\hline Errors and omissions & 0.3 & 0.6 & 0.2 & 0.2 & 0.0 & 0.0 & -0.1 & -0.1 & 0.0 & 0.0 & 0.0 & 0.0 \\
\hline Financing gap & 0.0 & 0.0 & 0.0 & 0.0 & 0.0 & 0.0 & 0.0 & 0.0 & 0.0 & 0.0 & 0.0 & 0.0 \\
\hline Memorandum items: & \multicolumn{12}{|c|}{ (In percent of GDP, unless otherwise notified) } \\
\hline Airport travel tax earmarked for new airport (RDIA) & & & & & & & & & & 0.3 & & 0.3 \\
\hline Basic fiscal balance (WAEMU definition) $7 /$ & -1.2 & -1.3 & 1.9 & 2.0 & 0.6 & 0.6 & 0.0 & -0.2 & -1.1 & -0.8 & 0.1 & -1.5 \\
\hline HIPC Initiative expenditure & 0.5 & 0.5 & 0.1 & 0.1 & 0.8 & 0.8 & 0.7 & 0.7 & 1.4 & 1.6 & 0.7 & 1.1 \\
\hline Health expenditure & 2.1 & 2.1 & 1.7 & 1.7 & 1.8 & 1.8 & 2.1 & 2.1 & $\ldots$ & $\ldots$ & $\ldots$ & $\ldots$ \\
\hline Education expenditure & 4.3 & 4.3 & 4.4 & 4.4 & 5.0 & 5.0 & 4.9 & 4.9 & $\ldots$ & & $\ldots$ & $\ldots$ \\
\hline Wages and salaries (in percent of fiscal revenue) & 29.4 & 29.4 & 30.0 & 30.0 & 28.3 & 28.3 & 28.0 & 28.0 & 29.4 & 29.0 & 29.5 & 28.5 \\
\hline Gross domestic product (in billions of CFA francs) & 3,343 & 3,343 & 3,473 & 3,473 & 3,725 & 3,725 & 4,029 & 4,029 & 4,364 & 4,346 & 4,682 & 4,673 \\
\hline
\end{tabular}

Sources: Senegalese authorities; and staff estimates and projections.

1/ Before methodological changes: see Table 5, footnote 1 .

2/ After methodological changes: see Table 5, footnote 2 .

3/ Staff Report for the 2004 Article IV consultation and the second review.

4/ Local governments, autonomous public sector entities (e.g. hospitals, universities), and the civil servants' pension fund (FNR).

$5 /$ Defined as total revenue and grants minus total expenditure and net lending, excluding interest expenditure.

6/ Defined as total revenue minus total expenditure and net lending, excluding externally financed capital expenditure, on-lending

cost of structural reforms and HIPC expenditure.

7/ Defined as total revenue minus total expenditure and net lending, excluding externally financed capital expenditure, and on-lending. 
Table 7. Senegal: Quarterly Government Financial Operations, 2005

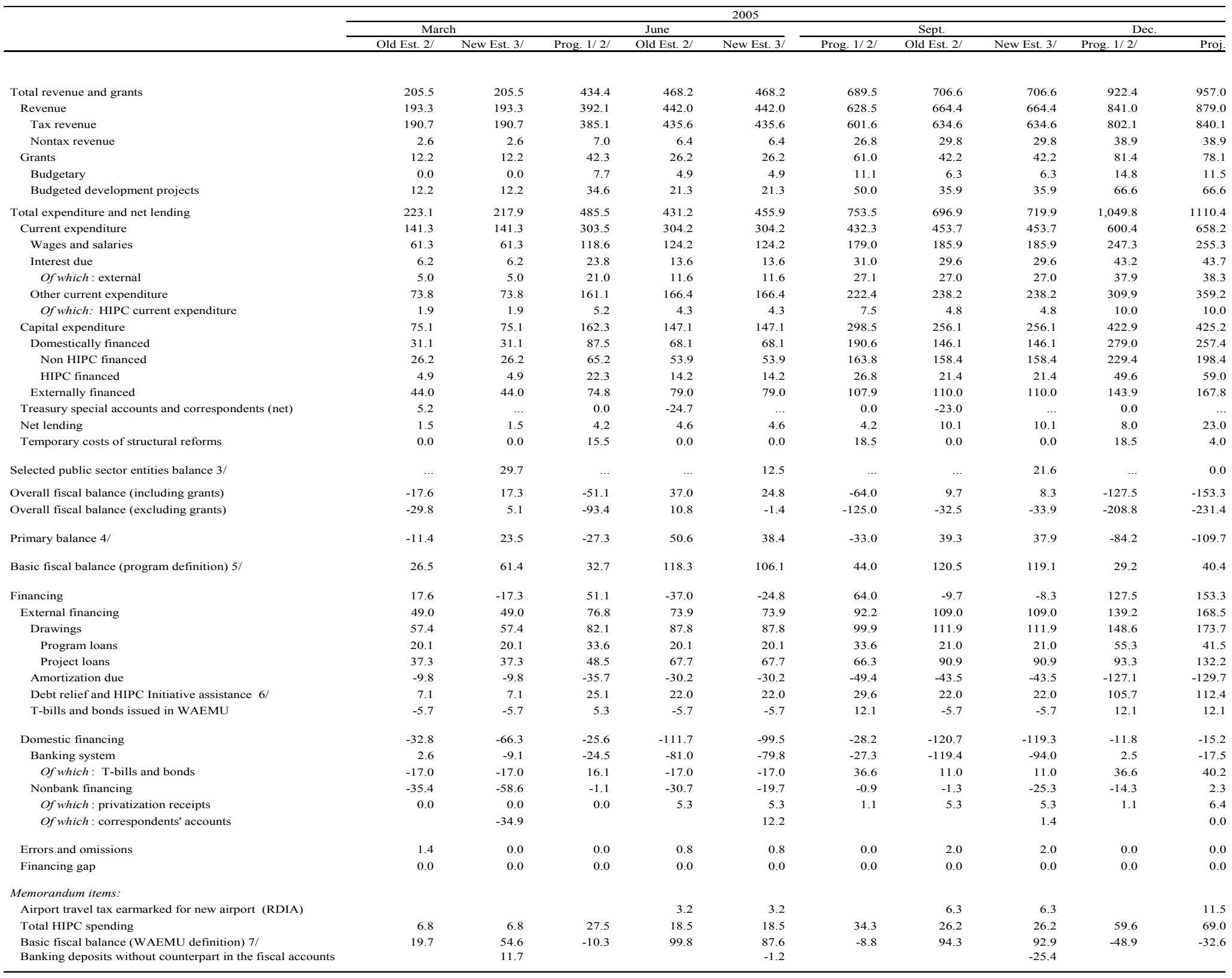

Sources: Senegalese authorities; and staff estimates and projections.

1/ Staff Report for the 2004 Article IV consultation and the second review.

2/ Before methodological changes: see Table 5, footnote 1 .

3/ After methodological changes: see Table 5 , footnote 2 .

4/ Local governments, autonomous public sector entities (e.g. hospitals, universities), and the civil servants' pension fund (FNR)

Defined as total revenue and grants minus total expenditure and net lending, excluding interest expenditure.

6/ Defined as total revenue minus total expenditure and net lending, excluding externally financed caphe

8/ Defined as total revenue minus total expenditure and net lending, excluding externally financed capital expenditure, and on-lending. 
Table 8. Senegal: Monetary Survey, 2001-06

\begin{tabular}{|c|c|c|c|c|c|c|c|c|c|}
\hline & \multirow[t]{2}{*}{2001} & \multirow[t]{2}{*}{2002} & \multirow[t]{2}{*}{2003} & \multirow{2}{*}{$\begin{array}{l}2004 \\
\text { Est. }\end{array}$} & \multicolumn{3}{|c|}{2005} & \multicolumn{2}{|c|}{2006} \\
\hline & & & & & Sept. & Prog. 1/ & Proj. & Proj. 1/ & Rev. proj. \\
\hline & \multicolumn{9}{|c|}{ (In billions of CFA francs) } \\
\hline Net foreign assets & 169.6 & 296.8 & 550.8 & 676.3 & 711.7 & 513.2 & 739.2 & 540.5 & 783.3 \\
\hline Central Bank of West African States (BCEAO) & 66.7 & 137.7 & 351.6 & 477.1 & 508.3 & 289.5 & 530.1 & 305.6 & 563.7 \\
\hline Commercial banks & 102.9 & 159.1 & 199.2 & 199.2 & 203.4 & 223.8 & 209.2 & 235.0 & 219.6 \\
\hline Net domestic assets & 735.6 & 677.3 & 729.8 & 769.5 & 781.0 & 786.7 & 938.6 & 841.7 & $1,078.3$ \\
\hline Net domestic credit & 837.4 & 792.9 & 848.8 & 880.9 & 925.7 & 913.5 & 1047.3 & 970.2 & $1,187.7$ \\
\hline Net credit to the government & 182.0 & 106.5 & 64.2 & 24.0 & -95.4 & 35.1 & 6.5 & 23.9 & 47.5 \\
\hline Central bank & 221.0 & 188.7 & 175.5 & 107.9 & 1.4 & 104.7 & 53.8 & 90.7 & 79.8 \\
\hline Commercial banks & -42.0 & -83.0 & -117.2 & -93.7 & -104.3 & -75.5 & -57.1 & -72.7 & -42.1 \\
\hline Other institutions & 3.0 & 0.8 & 5.9 & 9.8 & 7.5 & 5.9 & 9.8 & 5.9 & 9.8 \\
\hline Credit to the economy & 655.5 & 686.4 & 784.6 & 856.9 & 1021.1 & 878.3 & 1040.8 & 946.3 & $1,140.2$ \\
\hline Of which : crop credit & 5.0 & 0.9 & 3.6 & 14.0 & 20.5 & 2.2 & 15.1 & 2.4 & 16.2 \\
\hline Other items (net) & -101.8 & -115.6 & -119.0 & -111.4 & -144.7 & -126.8 & -108.7 & -128.5 & -109.4 \\
\hline Broad money (M2) & 905.2 & 974.1 & $1,280.6$ & $1,445.8$ & $1,492.7$ & $1,299.9$ & $1,677.8$ & $1,382.3$ & $1,861.6$ \\
\hline Currency outside banks & 217.8 & 192.6 & 337.5 & 344.3 & 323.2 & 199.2 & 349.7 & 211.7 & 372.5 \\
\hline Total deposits & 687.4 & 781.5 & 943.1 & $1,101.5$ & $1,169.5$ & $1,100.8$ & $1,328.1$ & $1,170.6$ & $1,489.1$ \\
\hline Demand deposits & 323.5 & 372.6 & 495.4 & 563.1 & 584.1 & 524.8 & 833.5 & 558.1 & 934.6 \\
\hline \multirow[t]{2}{*}{ Time deposits } & 363.9 & 408.9 & 447.7 & 538.4 & 585.4 & 576.0 & 494.6 & 612.5 & 554.5 \\
\hline & \multicolumn{9}{|c|}{ (Change in percentage of beginning-of-period broad money stock) } \\
\hline Net foreign assets & 10.2 & 14.1 & 26.1 & 9.8 & 2.4 & 4.7 & 4.4 & 2.1 & 2.6 \\
\hline BCEAO & 9.1 & 7.8 & 22.0 & 9.8 & 2.2 & 3.8 & 3.7 & 1.2 & 2.0 \\
\hline Commercial banks & 1.1 & 6.2 & 4.1 & 0.0 & 0.3 & 0.8 & 0.7 & 0.9 & 0.6 \\
\hline Net domestic assets & 4.3 & -6.4 & 5.4 & 3.1 & 0.8 & 3.8 & 11.7 & 4.2 & 8.3 \\
\hline Net credit to the government & 2.7 & -8.3 & -4.3 & -3.1 & -8.3 & 0.2 & -1.2 & -0.9 & 2.4 \\
\hline Credit to the economy & 3.8 & 3.4 & 10.1 & 5.6 & 11.4 & 4.0 & 12.7 & 5.2 & 5.9 \\
\hline Other items (net) & -2.3 & -1.5 & -0.3 & 0.6 & -2.3 & -0.4 & 0.2 & -0.1 & 0.0 \\
\hline Broad money (M2) & 14.5 & 7.6 & 31.5 & 12.9 & 3.2 & 8.5 & 16.0 & 6.3 & 11.0 \\
\hline Memorandum items: & \multicolumn{9}{|c|}{ (in units indicated) } \\
\hline Velocity (GDP/M2; end of period) & 3.7 & 3.6 & 2.9 & 2.8 & 2.9 & 3.4 & 2.6 & 3.4 & 2.5 \\
\hline Nominal GDP growth (percentage growth) & 4.7 & 3.9 & 7.3 & 8.2 & 7.9 & 8.5 & 7.9 & 7.3 & 7.5 \\
\hline Credit to the economy (percentage growth) & 4.9 & 4.7 & 14.3 & 9.2 & 19.2 & 5.7 & 21.5 & 7.7 & 9.5 \\
\hline Credit to the economy/GDP (in percent) & 19.6 & 19.3 & 21.1 & 21.3 & 25.3 & 20.1 & 24.0 & 20.2 & 24.4 \\
\hline $\begin{array}{l}\text { Variation of net credit to the government } \\
\text { (since the beginning of the year; in billions of CFA francs) }\end{array}$ & 21.8 & -75.4 & -42.3 & -40.2 & -119.4 & 2.5 & -17.5 & -11.3 & 41.0 \\
\hline
\end{tabular}

Senrces: Senalese authorities; and staff estimates and projections.

1/ Staff Report for the 2004 Article IV consultation and the second review. 
Table 9. Senegal: Balance of Payments, 2001-06

\begin{tabular}{|c|c|c|c|c|c|c|c|c|}
\hline & \multirow[t]{2}{*}{2001} & \multirow[t]{2}{*}{2002} & \multirow[t]{2}{*}{2003} & \multirow{2}{*}{$\begin{array}{r}2004 \\
\text { Est. }\end{array}$} & \multicolumn{2}{|c|}{2005} & \multicolumn{2}{|c|}{2006} \\
\hline & & & & & Prog. 1/ & Proj. & Proj. 1/ & $\overline{\text { v. proj. }}$ \\
\hline & \multicolumn{8}{|c|}{ (In billions of CFA francs, unless otherwise indicated) } \\
\hline Current account & -156 & -207 & -245 & -270 & -236 & -331 & -238 & -356 \\
\hline Balance on goods & -312 & -375 & -470 & -533 & -466 & -591 & -492 & -632 \\
\hline Exports, f.o.b. & 735 & 743 & 731 & 775 & 815 & 841 & 860 & 896 \\
\hline Imports, f.o.b. & $-1,047$ & $-1,118$ & $-1,200$ & $-1,308$ & $-1,282$ & $-1,432$ & $-1,352$ & $-1,528$ \\
\hline Services and incomes (net) & -67 & -92 & -87 & -98 & -97 & -102 & -94 & -99 \\
\hline Credits & 341 & 365 & 400 & 401 & 414 & 417 & 429 & 434 \\
\hline Of which: tourism & 128 & 132 & 121 & 128 & 134 & 135 & 140 & 141 \\
\hline Debits & -408 & -457 & -487 & -499 & -512 & -518 & -522 & -532 \\
\hline Of which: interest on public debt & -25 & -36 & -41 & -42 & -38 & -39 & -36 & -38 \\
\hline Unrequited current transfers (net) & 223 & 260 & 312 & 362 & 327 & 361 & 348 & 374 \\
\hline Private (net) & 173 & 192 & 241 & 287 & 260 & 297 & 270 & 309 \\
\hline Public (net) & 50 & 68 & 71 & 75 & 67 & 64 & 78 & 65 \\
\hline Of which: budgetary grants & 0 & 2 & 19 & 19 & 15 & 11 & 29 & 16 \\
\hline Capital and financial account & 218 & 316 & 459 & 294 & 186 & 282 & 176 & 306 \\
\hline Capital account & 85 & 66 & 67 & 77 & 74 & 74 & 80 & 80 \\
\hline Private capital transfers & 2 & 6 & 7 & 7 & 7 & 7 & 8 & 8 \\
\hline Project grants & 62 & 60 & 60 & 70 & 67 & 67 & 72 & 72 \\
\hline Debt cancellation & 21 & 0 & 0 & 0 & 0 & 0 & 0 & 0 \\
\hline Financial account & 133 & 249 & 392 & 217 & 112 & 208 & 96 & 226 \\
\hline Direct investment & 29 & 31 & 29 & 58 & 63 & 59 & 70 & 75 \\
\hline Portfolio investment & 10 & 2 & 13 & 11 & 3 & 19 & -20 & 22 \\
\hline Other investment & 94 & 217 & 350 & 149 & 46 & 130 & 46 & 129 \\
\hline Public sector (net) & 55 & 43 & 20 & 37 & 24 & 47 & 1 & 33 \\
\hline Of which: disbursements & 117 & 121 & 91 & 162 & 151 & 175 & 130 & 164 \\
\hline program loans & 61 & 42 & 0 & 13 & 55 & 41 & 27 & 32 \\
\hline project loans & 43 & 71 & 91 & 144 & 93 & 132 & 101 & 132 \\
\hline other & 5 & 0 & 0 & 2 & 2 & 1 & 2 & 0 \\
\hline amortization & -62 & -79 & -72 & -126 & -127 & -129 & -128 & -132 \\
\hline Private sector (net) & 41 & 128 & 133 & 90 & 22 & 83 & 45 & 96 \\
\hline Errors and omissions & -2 & 46 & 197 & 23 & 0 & 0 & 0 & 0 \\
\hline Overall balance & 62 & 109 & 214 & 24 & -50 & -50 & -62 & -50 \\
\hline Financing & -62 & -109 & -214 & -24 & 50 & 50 & 62 & 50 \\
\hline Net foreign assets (BCEAO) & -72 & -71 & -214 & -126 & -46 & -53 & -16 & -34 \\
\hline Net use of Fund resources & -2 & -13 & -20 & -23 & -17 & -22 & -13 & -10 \\
\hline Purchases & 22 & 8 & 3 & 3 & 6 & 3 & 7 & 11 \\
\hline Repurchases & -23 & -21 & -23 & -26 & -24 & -24 & -20 & -21 \\
\hline Other & -71 & -58 & -194 & -102 & -29 & -31 & -3 & -24 \\
\hline Deposit money banks & -9 & -56 & -40 & 0 & -10 & -10 & -11 & -10 \\
\hline Payments arrears (reduction -) & 0 & 0 & 0 & 0 & 0 & 0 & 0 & 0 \\
\hline Exceptional financing 2/ & 19 & 18 & 40 & 101 & 106 & 112 & 89 & 94 \\
\hline Residual financing gap & 0 & 0 & 0 & 0 & 0 & 0 & 0 & 0 \\
\hline \multicolumn{9}{|l|}{ Memorandum items: } \\
\hline \multicolumn{9}{|l|}{ Current account balance } \\
\hline As percentage of GDP (incl. current official transfers) & -4.7 & -6.0 & -6.6 & -6.7 & -5.4 & -7.6 & -5.1 & -7.6 \\
\hline As percentage of GDP (excl. current official transfers) & -6.3 & -8.0 & -8.6 & -8.7 & -7.1 & -9.2 & -6.9 & -9.1 \\
\hline Gross official reserves (in billions of CFA francs) & 339 & 404 & 577 & 668 & 473 & 707 & 480 & 732 \\
\hline (in months of imports of GNFS) & 3.0 & 3.3 & 4.5 & 4.8 & 3.4 & 4.7 & 3.3 & 4.6 \\
\hline Nominal GDP (in billions of CFA francs) & 3,343 & 3,473 & 3,725 & 4,029 & 4,364 & 4,346 & 4,682 & 4,673 \\
\hline
\end{tabular}

Sources: Central Bank of West African States (BCEAO); and staff estimates and projections.

1/ Staff Report for the 2004 Article IV consultation and the second review.

2/ HIPC Initiative debt relief granted by the IMF is recorded as a grant, and that granted by the World Bank, the African Development Bank,

Paris Club creditors and Kuwait is recorded as exceptional financing. 
Table 10. Senegal: Selected Medium-Term Economic and Financial Indicators, 2003-10

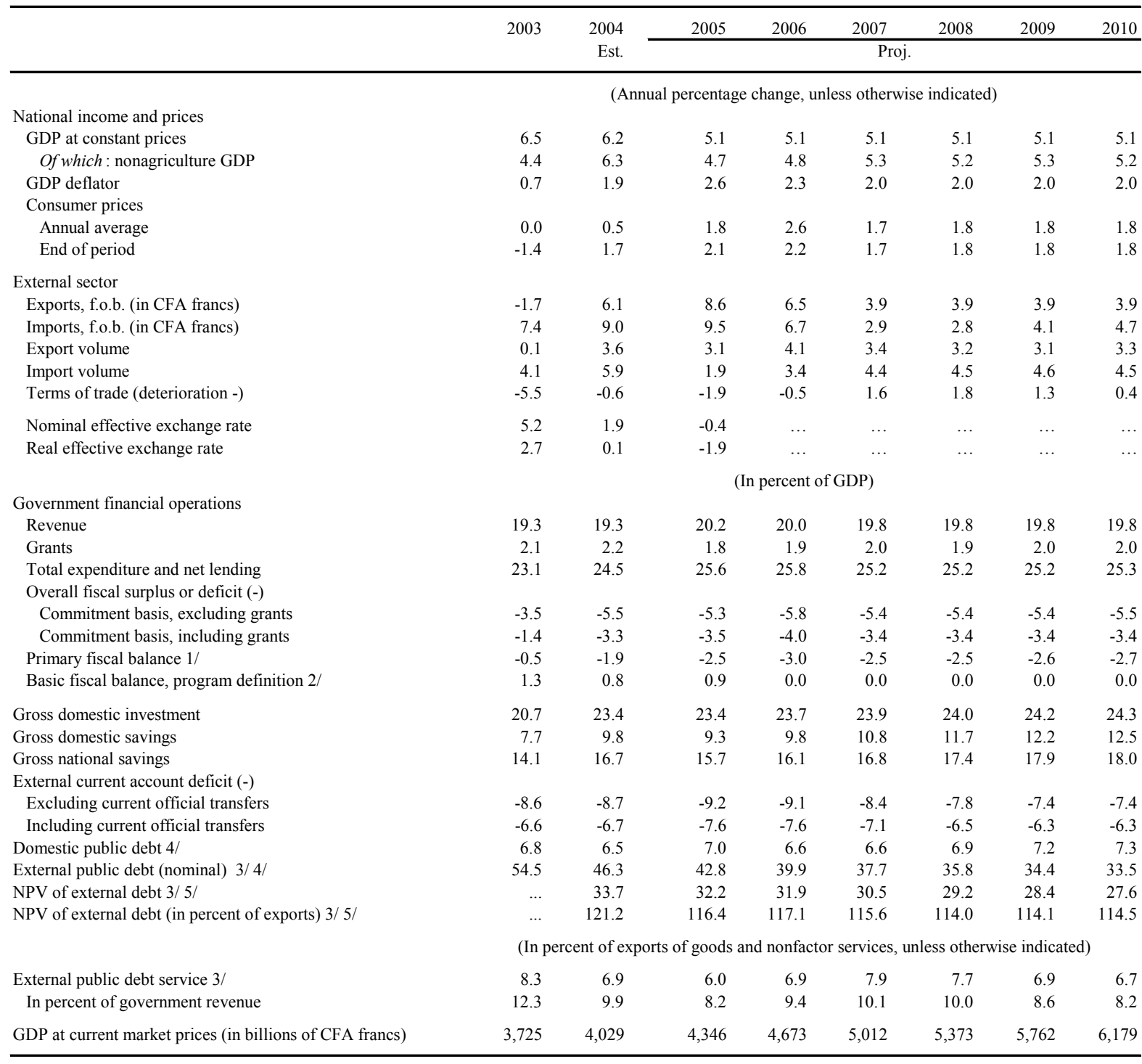

Sources: Senegalese authorities; and staff estimates and projections.

1/ Defined as total revenue and grants minus total expenditure and net lending, excluding interest expenditure.

2/ Defined as total revenue minus total expenditure and net lending, excluding externally financed capital expenditure, on-lending,

temporary costs of structural reforms and expenditure financed with HIPC Initiative assistance.

3/ After HIPC debt relief.

4/ Debt outstanding at year-end.

5/ Including programmed new debt. 
Table 11 Senegal: Medium-Term Balance of Payments, 2003-10

\begin{tabular}{|c|c|c|c|c|c|c|c|c|}
\hline & 2003 & 2004 & 2005 & 2006 & 2007 & 2008 & 2009 & 2010 \\
\hline & & Est. & \multicolumn{6}{|c|}{ Proj. } \\
\hline & \multicolumn{8}{|c|}{ (In billions of CFA francs, unless otherwise indicated) } \\
\hline Current account & -245 & -270 & -331 & -356 & -355 & -351 & -361 & -390 \\
\hline Balance on goods & -470 & -533 & -591 & -632 & -641 & -648 & -676 & -715 \\
\hline Exports, f.o.b. & 731 & 775 & 841 & 896 & 931 & 968 & 1006 & 1045 \\
\hline Imports, f.o.b. & $-1,200$ & $-1,308$ & $-1,432$ & $-1,528$ & $-1,572$ & $-1,616$ & $-1,682$ & $-1,760$ \\
\hline Services and incomes (net) & -87 & -98 & -102 & -99 & -97 & -98 & -95 & -99 \\
\hline Credits & 400 & 401 & 417 & 434 & 451 & 470 & 489 & 509 \\
\hline Debits & -487 & -499 & -518 & -532 & -548 & -568 & -583 & -608 \\
\hline Unrequited current transfers (net) & 312 & 362 & 361 & 374 & 382 & 395 & 409 & 424 \\
\hline Private (net) & 241 & 287 & 297 & 309 & 321 & 334 & 347 & 360 \\
\hline Public (net) & 71 & 75 & 64 & 65 & 61 & 61 & 63 & 64 \\
\hline Capital and financial account & 459 & 294 & 282 & 306 & 342 & 294 & 336 & 368 \\
\hline Capital account & 67 & 77 & 74 & 80 & 90 & 97 & 106 & 116 \\
\hline Private capital transfers & 7 & 7 & 7 & 8 & 8 & 9 & 9 & 9 \\
\hline Project grants & 60 & 70 & 67 & 72 & 82 & 88 & 97 & 107 \\
\hline Financial account & 392 & 217 & 208 & 226 & 252 & 198 & 230 & 252 \\
\hline Direct investment & 29 & 58 & 59 & 75 & 80 & 84 & 87 & 91 \\
\hline Portfolio investment & 13 & 11 & 19 & 22 & 17 & 17 & 27 & 26 \\
\hline Other investment & 350 & 149 & 130 & 129 & 155 & 97 & 116 & 135 \\
\hline Public sector (net) & 20 & 37 & 47 & 33 & 55 & 62 & 83 & 103 \\
\hline Of which: disbursements & 91 & 162 & 175 & 164 & 186 & 200 & 224 & 245 \\
\hline program loans & 0 & 13 & 41 & 32 & 34 & 37 & 39 & 42 \\
\hline project loans & 91 & 144 & 132 & 132 & 152 & 163 & 184 & 203 \\
\hline other & 0 & 2 & 1 & 0 & 0 & 0 & 0 & 0 \\
\hline amortization & -72 & -126 & -129 & -132 & -132 & -139 & -141 & -141 \\
\hline Private sector (net) & 133 & 90 & 83 & 96 & 99 & 35 & 33 & 32 \\
\hline Errors and omissions & 197 & 23 & 0 & 0 & 0 & 0 & 0 & 0 \\
\hline Overall balance & 214 & 24 & -50 & -50 & -13 & -56 & -24 & -22 \\
\hline Financing & -214 & -24 & 50 & 50 & 13 & 56 & 24 & 22 \\
\hline Net foreign assets (BCEAO) & -214 & -126 & -53 & -34 & -57 & -16 & -48 & -45 \\
\hline Deposit money bank & -40 & 0 & -10 & -10 & -11 & -12 & -12 & -13 \\
\hline Exceptional financing 1/ & 40 & 101 & 112 & 94 & 81 & 84 & 84 & 80 \\
\hline Residual financing gap & 0 & 0 & 0 & 0 & 0 & 0 & 0 & 0 \\
\hline \multicolumn{9}{|l|}{ Memorandum items: } \\
\hline \multicolumn{9}{|l|}{ Current account balance } \\
\hline As percentage of GDP (incl. current official transfers) & -6.6 & -6.7 & -7.6 & -7.6 & -7.1 & -6.5 & -6.3 & -6.3 \\
\hline As percentage of GDP (excl. current official transfers) & -8.6 & -8.7 & -9.2 & -9.1 & -8.4 & -7.8 & -7.4 & -7.4 \\
\hline Gross official reserves & 577 & 668 & 707 & 732 & 772 & 775 & 813 & 852 \\
\hline (in months of imports of GNFS) & 4.5 & 4.8 & 4.7 & 4.6 & 4.7 & 4.6 & 4.6 & 4.6 \\
\hline Nominal GDP (in billions of CFA francs) & 3,725 & 4,029 & 4,346 & 4,673 & 5,012 & 5,373 & 5,762 & 6,179 \\
\hline
\end{tabular}

Sources: Central Bank of West African States (BCEAO); and staff estimates and projections.

1/ HIPC Initiative debt relief is recorded as a grant for the IMF, and as exceptional financing for the World Bank, the African Development Bank, Paris Club creditors and Kuwait. 
Table 12. Senegal: Fund Position During the Period of the PRGF Arrangement, 2003-06

\begin{tabular}{|c|c|c|c|c|c|c|c|c|c|c|c|c|c|c|c|c|c|c|c|c|}
\hline & \multicolumn{5}{|c|}{2003} & \multicolumn{5}{|c|}{2004} & \multicolumn{5}{|c|}{2005} & \multicolumn{5}{|c|}{2006} \\
\hline & $\begin{array}{l}\text { Jan.- } \\
\text { Mar. }\end{array}$ & $\begin{array}{l}\text { Apr.- } \\
\text { June }\end{array}$ & $\begin{array}{l}\text { Jul.- } \\
\text { Sep. }\end{array}$ & $\begin{array}{l}\text { Oct.- } \\
\text { Dec. }\end{array}$ & TOTAL & $\begin{array}{l}\text { Jan.- } \\
\text { Mar. }\end{array}$ & $\begin{array}{l}\text { Apr.- } \\
\text { June }\end{array}$ & $\begin{array}{l}\text { Jul.- } \\
\text { Sep. }\end{array}$ & $\begin{array}{l}\text { Oct.- } \\
\text { Dec. }\end{array}$ & TOTAL & $\begin{array}{l}\text { Jan.- } \\
\text { Mar. }\end{array}$ & $\begin{array}{l}\text { Apr.- } \\
\text { June }\end{array}$ & $\begin{array}{l}\text { Jul.- } \\
\text { Sep. }\end{array}$ & $\begin{array}{l}\text { Oct.- } \\
\text { Dec. }\end{array}$ & TOTAL & $\begin{array}{l}\text { Jan.- } \\
\text { Mar. }\end{array}$ & $\begin{array}{l}\text { Apr.- } \\
\text { June }\end{array}$ & $\begin{array}{l}\text { Jul.- } \\
\text { Sep. }\end{array}$ & $\begin{array}{l}\text { Oct.- } \\
\text { Dec. }\end{array}$ & TOTAL \\
\hline & \multicolumn{20}{|c|}{ (In millions of SDRs) } \\
\hline Disbursements under PRGF & 0.0 & 3.5 & 0.0 & 0.0 & 3.5 & 3.5 & 0.0 & 0.0 & 0.0 & 3.5 & 3.5 & 0.0 & 0.0 & 0.0 & 3.5 & 13.9 & 0.0 & 0.0 & 0.0 & 13.9 \\
\hline IMF HIPC assistance & 0.0 & 1.4 & 1.9 & 1.8 & 5.1 & 0.5 & 2.8 & 3.5 & 3.2 & 10.0 & 3.6 & 2.6 & 2.1 & 1.7 & 10.0 & 2.8 & 0.6 & 1.2 & 0.6 & 5.2 \\
\hline Repurchases/repayments & 7.6 & 5.5 & 7.6 & 7.3 & 27.9 & 7.6 & 9.0 & 7.6 & 9.0 & 33.3 & 7.4 & 9.0 & 7.4 & 7.4 & 31.2 & 7.4 & 5.0 & 8.8 & 5.0 & 26.2 \\
\hline Ordinary resources & 0.0 & 0.0 & 0.0 & 0.0 & 0.0 & 0.0 & 0.0 & 0.0 & 0.0 & 0.0 & 0.0 & 0.0 & 0.0 & 0.0 & 0.0 & 0.0 & 0.0 & 0.0 & 0.0 & 0.0 \\
\hline $\mathrm{SAF} / \mathrm{PRGF}$ and SBA & 7.6 & 5.5 & 7.6 & 7.3 & 27.9 & 7.6 & 9.0 & 7.6 & 9.0 & 33.3 & 7.4 & 9.0 & 7.4 & 7.4 & 31.2 & 7.4 & 5.0 & 8.8 & 5.0 & 26.2 \\
\hline Charges and interests & 0.1 & 0.5 & 0.1 & 0.5 & 1.2 & 0.1 & 0.5 & 0.1 & 0.5 & 1.1 & 0.1 & 0.4 & 0.1 & 0.4 & 1.1 & 0.1 & 0.4 & 0.1 & 0.3 & 1.0 \\
\hline Ordinary resources & 0.0 & 0.0 & 0.0 & 0.0 & 0.0 & 0.0 & 0.0 & 0.0 & 0.0 & 0.0 & 0.0 & 0.0 & 0.0 & 0.0 & 0.0 & 0.0 & 0.0 & 0.0 & 0.0 & 0.0 \\
\hline $\mathrm{SAF} / \mathrm{PRGF}$ and SBA & 0.0 & 0.4 & 0.0 & 0.4 & 0.9 & 0.0 & 0.4 & 0.0 & 0.4 & 0.7 & 0.0 & 0.3 & 0.0 & 0.3 & 0.6 & 0.0 & 0.2 & 0.0 & 0.2 & 0.4 \\
\hline \multicolumn{21}{|l|}{ Total Fund credit outstanding } \\
\hline (end of period) & 178.2 & 176.2 & 168.5 & 161.3 & 161.3 & 157.1 & 148.1 & 140.5 & 131.5 & 131.5 & 127.6 & 118.5 & 111.2 & 103.8 & 103.8 & 110.3 & 105.3 & 96.5 & 91.5 & 91.5 \\
\hline Ordinary resources & 0.0 & 0.0 & 0.0 & 0.0 & 0.0 & 0.0 & 0.0 & 0.0 & 0.0 & 0.0 & 0.0 & 0.0 & 0.0 & 0.0 & 0.0 & 0.0 & 0.0 & 0.0 & 0.0 & 0.0 \\
\hline SAF / PRGF and SBA & 178.2 & 176.2 & 168.5 & 161.3 & 161.3 & 157.1 & 148.1 & 140.5 & 131.5 & 131.5 & 127.6 & 118.5 & 111.2 & 103.8 & 103.8 & 110.3 & 105.3 & 96.5 & 91.5 & 91.5 \\
\hline Quota & 161.8 & 161.8 & 161.8 & 161.8 & 161.8 & 161.8 & 161.8 & 161.8 & 161.8 & 161.8 & 161.8 & 161.8 & 161.8 & 161.8 & 161.8 & 161.8 & 161.8 & 161.8 & 161.8 & 161.8 \\
\hline & \multicolumn{20}{|c|}{ (In percent of quota) } \\
\hline \multicolumn{21}{|l|}{ Total Fund credit outstanding } \\
\hline (end of period) & 110.1 & 108.9 & 104.2 & 99.7 & 99.7 & 97.1 & 91.5 & 86.8 & 81.3 & 81.3 & 78.8 & 73.3 & 68.7 & 64.1 & 64.1 & 68.2 & 65.1 & 59.6 & 56.5 & 56.5 \\
\hline Ordinary resources & 0.0 & 0.0 & 0.0 & 0.0 & 0.0 & 0.0 & 0.0 & 0.0 & 0.0 & 0.0 & 0.0 & 0.0 & 0.0 & 0.0 & 0.0 & 0.0 & 0.0 & 0.0 & 0.0 & 0.0 \\
\hline $\mathrm{SAF} / \mathrm{PRGF}$ & 110.1 & 108.9 & 104.2 & 99.7 & 99.7 & 97.1 & 91.5 & 86.8 & 81.3 & 81.3 & 78.8 & 73.3 & 68.7 & 64.1 & 64.1 & 68.2 & 65.1 & 59.6 & 56.5 & 56.5 \\
\hline
\end{tabular}

Sources: International Monetary Fund, Finance Department; and staff projections. 
Table 13. Financial Soundness Indicators for the Banking Sector, 2000-05

\begin{tabular}{|c|c|c|c|c|c|c|}
\hline & 2000 & 2001 & 2002 & 2003 & 2004 & \multirow{2}{*}{$\begin{array}{r}2005 \\
\text { Sept. }\end{array}$} \\
\hline & \multicolumn{5}{|c|}{ December } & \\
\hline & \multicolumn{6}{|c|}{ (In percent, unless otherwise indicated) } \\
\hline \multicolumn{7}{|l|}{ Capital Adequacy } \\
\hline Capital to risk weighted assets & 20.6 & 16.8 & 16.0 & 12.1 & 11.9 & 11.6 \\
\hline Regulatory capital to risk weighted assets & 20.6 & 16.8 & 15.5 & 11.7 & 11.5 & 11.4 \\
\hline Capital to total assets & 9.9 & 9.7 & 10.3 & 7.8 & 7.7 & 8.1 \\
\hline \multicolumn{7}{|l|}{ Asset composition and quality } \\
\hline Total loans to total assets & 62.8 & 59.4 & 58.3 & 59.6 & 57.1 & 62.5 \\
\hline $\begin{array}{l}\text { Concentration: loans to } 5 \text { largest borrowers to capital } \\
\text { Sectoral distribution of loans }\end{array}$ & & 143.1 \\
\hline Industrial & 31.3 & 33.1 & 36.4 & 41.1 & 33.6 & 33.6 \\
\hline Retail and wholesale trade & 28.6 & 23.6 & 22.2 & 19.9 & 19.3 & 17.5 \\
\hline Services, transport and communications & 14.9 & 16.3 & 17.5 & 17.2 & 27.4 & 28.9 \\
\hline Gross NPLs to total loans & 18.1 & 17.8 & 18.5 & 13.3 & 12.6 & 13.4 \\
\hline Provisions to NPLs & 67.6 & 70.2 & 70.5 & 75.3 & 75.7 & 62.9 \\
\hline NPLs net of provisions to total loans & 6.7 & 5.6 & 5.5 & 3.3 & 3.4 & 5.4 \\
\hline NPLs net of provisions to capital & 51.3 & 34.3 & 30.7 & 27.8 & 25.1 & 41.9 \\
\hline \multicolumn{7}{|l|}{ Earnings and Profitability } \\
\hline Average cost of borrowed funds & 2.2 & 2.4 & 2.2 & 1.8 & 1.9 & $\ldots$ \\
\hline Average interest rate on loans & 10.1 & 10.1 & 9.7 & 8.7 & 8.3 & $\ldots$ \\
\hline Average interest margin ${ }^{1 /}$ & 7.9 & 7.7 & 7.6 & 6.7 & 6.4 & $\ldots$ \\
\hline After-tax return on average assets & 1.7 & 1.6 & 1.8 & 1.8 & 1.8 & $\ldots$ \\
\hline After-tax return on average equity & 20.3 & 18.6 & 21.1 & $22.1^{2 /}$ & 17.6 & $\ldots$ \\
\hline Noninterest expenses/net banking income & 45.7 & 44.5 & 45.4 & 48.9 & 48.7 & $\ldots$ \\
\hline Salaries and wages/net banking income & 21.3 & 19.9 & 20.6 & 21.8 & 21.5 & $\ldots$ \\
\hline \multicolumn{7}{|l|}{ Liquidity } \\
\hline Liquid assets to total assets ${ }^{2 /}$ & $\ldots$ & $\ldots$ & 65.1 & 66.5 & 66.4 & $\ldots$ \\
\hline Liquid assets to total deposits ${ }^{3 /}$ & & & 82.9 & 81.0 & 82.0 & \\
\hline Total deposits to total liabilities & 81.5 & 75.5 & 78.5 & 82.0 & 79.6 & 77.8 \\
\hline
\end{tabular}

Source: Senegalese authorities.

1/ Excluding the tax on banking operations.

2/ Estimate.

2/ As of March 2004. 
Table 14. Senegal: Schedule of Projected Reviews and Disbursements

Under the PRGF Arrangement, 2003-06

\begin{tabular}{|c|c|c|}
\hline Date & Action & Disbursement \\
\hline April 2003 & $\begin{array}{l}\text { Executive Board consideration of } \\
\text { request for a three-year arrangement } \\
\text { under the Poverty Reduction and } \\
\text { Growth Facility (PRGF) and } \\
\text { conclusion of the } 2002 \text { Article IV } \\
\text { consultation. }\end{array}$ & SDR 3.47 million \\
\hline End-June 2003 & $\begin{array}{l}\text { Quantitative performance criteria. } \\
\text { Test date for the first review. }\end{array}$ & \\
\hline End-December 2003 & $\begin{array}{l}\text { Quantitative performance criteria. } \\
\text { Test date for the second review. }\end{array}$ & \\
\hline February 2004 & $\begin{array}{l}\text { Executive Board consideration of } \\
\text { the first review under the PRGF } \\
\text { arrangement. }\end{array}$ & SDR 3.47 million \\
\hline March 2005 & $\begin{array}{l}\text { Executive Board consideration of } \\
\text { the second review under the PRGF } \\
\text { arrangement and conclusion of } \\
\text { the } 2004 \text { Article IV consultation. }\end{array}$ & SDR 3.47 million \\
\hline End-March 2005 & $\begin{array}{l}\text { Quantitative performance criteria. } \\
\text { Test date for the third review. }\end{array}$ & \\
\hline $\begin{array}{l}\text { End- } \\
\text { September } 2005\end{array}$ & $\begin{array}{l}\text { Quantitative performance criteria. } \\
\text { Test date for the fourth review. }\end{array}$ & \\
\hline January 2006 & $\begin{array}{l}\text { Executive Board consideration of } \\
\text { the third and fourth reviews under } \\
\text { the PRGF arrangement. }\end{array}$ & SDR 13.86 million \\
\hline
\end{tabular}




\section{Goal 1. Eradicate extreme poverty and hunger}

Target 1: Halve between 1990 and 2015, the proportion of people whose income is less than one dollar a day
1. Population below US\$1 a day (percent)
2. Poverty gap at US\$1 a day (percent)
3. Share of income or consumption held by poorest 20 (percent)

$\begin{array}{rr}45.4 & 22.3 \\ 20.0 & 5.7 \\ \ldots & 6.4\end{array}$

Target 2: Halve between 1990 and 2015, the proportion of people suffering hunger

4. Prevalence of child malnutrition (\% of children under 5 )

\section{Goal 2. Achieve universal primary education}

Target 3: Ensure that, by 2015, children will be able to complete a full course of primary schooling

6. Net primary enrollment ratio (percent of relevant age group)

7. Percentage of cohort reaching grade 5 (percent)

8. Youth literacy rate (\% ages $15-24)$

\section{Goal 3. Promote gender equality and empower women}

Target 4: Eliminate gender disparity in primary and secondary education preferably by 2005 and to all levels of education by 2015

9. Ratio of girls to boys in primary and secondary education (percent)

10. Ratio of young literate females to males (percent ages 15-24)

11. Share of women employed in the nonagricultural sector (percent)

12. Proportion of seats held by women in national parliament (percent)

Goal 4. Reduce child mortality

Target 5: Reduce by two-thirds, between 1990 and 2015, the under-five mortality rate

13. Under 5 mortality rate (per 1,000$)$

14. Infant mortality rate (per 1,000 live births)

15. Immunization, measles (percent of children under 12 months)

148.0

Goal 5. Improve maternal health

$\begin{array}{rrrrr}\ldots & 57.9 & 62.5 & 57.9 & {[100.0]} \\ \ldots & \ldots & 67.5 & \ldots & \\ 44.3 & 47.5 & 50.7 & 52.9 & \end{array}$

Target 6: Reduce by three-quarters, between 1990 and 2015, the maternal mortality rate

16. Maternal mortality ratio (modeled estimate, per 100,000 live births)

17. Births attended by skilled health staff (percent)

1200.0

$\begin{array}{rrrr}\ldots & 81.6 & 83.9 & 87.1 \\ 64.5 & 67.6 & 70.4 & 72.5 \\ \ldots & \ldots & \ldots & \ldots \\ \ldots & 12.0 & 12.0 & 19.0\end{array}$

[100.0] 19.0 
Table 15. Senegal: Millennium Development Goals (concluded)

\section{Goal 6. Combat HIV/AIDS, malaria and other diseases}

Target 7: Halt by 2015, and begin to reverse, the spread of HIV/AIDS

18. Prevalence of HIV (percent of population aged 15-24)

19. Contraceptive prevalence rate (percent of women aged 15-49)

20. Number of children orphaned by HIV/AIDS

.

$\cdots$

Target 8: Halt by 2015 , and begin to reverse, the incidence of malaria and

$$
\text { other major diseases }
$$

21. Malaria incidence (notified cases, per 100,000 people)

22. Malaria prevention (percent of population aged under 5 using insecticide-treated bed nets)

23. Tuberculosis incidence (per 100,000 people)

24. Tuberculosis cases detected under DOTS (percent)

$\begin{array}{rrrrr}\ldots & \ldots & \ldots & 11925.0 & \\ \ldots & \ldots & \ldots & 1.7 & \\ 200.2 & 213.1 & 223.2 & 233.9 & 245.1 \\ \ldots & 67.2 & 61.2 & 59.7 & 59.6\end{array}$

\section{Goal 7. Ensure environmental sustainability}

Target 9: Integrate the principles of sustainable development into policies and programs. Reverse the loss of environmental resources

25. Forest area ( $\%$ of total land area)

26. Nationally protected areas (\% of total land area)

27. GDP per unit of energy use (2000 PPP \$ per kg oil equivalent)

28. $\mathrm{CO} 2$ emissions (metric tons per capita)

29. Access to an improved water source (percent of population)

Target 11: Achieve significant improvement in life of at least 100 million slum dwellers by 2020

30. Access to improved sanitation (percent of population)

Target 10: Halve by 2015 proportion of people without access to safe drinking water

\section{Goal 8. Develop a Global Partnership for Development}

Target 12: Develop and implement strategies for productive work for youth

31. Youth unemployment rate (percent of total labor force ages 15-24)

32. Fixed line and mobile telephones (per 1,000 people)

33. Personal computers (per 1,000 people) 
Table 16. Senegal: Follow-up on HIPC Assistance

(As of December 15, 2005)

Assistance agreed at the decision point (June, 2000)
Interim

Assistance granted after the decision point
Assistance granted

after the completion

point (April, 2004)

Comments

CFAF 7.4 billion have been refunded, representing assistance from 4/1/2004 to $12 / 31 / 2004$

Agreement signed, May 20,2005

Refinancing assistance

Nordic Fund

Yes

\section{Paris Club Creditors}

France

Germany

Italy

Japan

Spain

United States

Norway

Denmark

The Netherlands

Belgium

Canada

United Kingdom

Sweden
Yes

Yes

Yes

Yes

Yes

Yes

Yes

Yes

Yes

Yes

Yes

Yes

Yes

yes yes

no yes

yes

yes

yes

yes

no

yes

yes

yes

no

no

yes

no

yes

yes

no

yes

yes

yes

yes

yes

yes

yes

yes

yes

yes

yes

no yes

yes

yes

no

\section{Bilateral creditors-Non Paris Club members}

$\begin{array}{llcr}\text { Kuwait } & \text { No } & \text { no } & \text { yes } \\ \text { Saudi Arabia } & \text { No } & \text { no } & \text { no } \\ \text { China } & \text { No } & \text { no } & \text { no } \\ \text { Taiwan, Prov.of China } & \text { n/a } & \text { n/a } & \text { no } \\ \text { Emirates } & \text { No } & \text { no } & \text { no } \\ \text { Algeria } & \text { No } & \text { no } & \text { no } \\ \text { Oman } & \text { No } & \text { no } & \text { no } \\ \text { Iraq } & \text { No } & \text { no } & \text { no }\end{array}$

Source: Senegalese authorities. 
Dear Mr. de Rato,

1. On April 28, 2003, the Executive Board of the International Monetary Fund approved a three-year arrangement under the Poverty Reduction and Growth Facility (PRGF) in the amount of SDR 24.27 million, in support of Senegal's economic growth and poverty reduction program for 2003-05. The first two reviews under this arrangement were completed on February 13, 2004 and March 8, 2005. The attached memorandum on economic and financial policies reviews the macroeconomic and financial performance and the implementation of economic policies in 2005. It also describes the economic policies and structural reforms that the government of Senegal will pursue in 2006.

2. As stated in paragraphs 4 and 5 of the memorandum, 11 of the 12 program's quantitative performance criteria at end-March 2005- the test date for the third review-and 8 of the 10 quantitative performance criteria at end-September 2005 - the test date for the fourth and final review-were met. However, owing to the rapid increase in oil prices in 2005, the financial situation of the electricity company SENELEC deteriorated and two continuous performance criteria (the ceiling on arrears of the company, and the ceiling on budgetary transfers to the company) were missed. The ceiling on no-bid contracts at endSeptember was also exceeded as extraordinary provisions of the Procurement Code were used in the case of a few large contracts of a strategic nature (paragraph 9 of the memorandum). The structural PC on elimination of a specific tax on vegetable oil was respected in December 2005.

3. The government has implemented a number of corrective measures and will implement others, as described in paragraphs 7 and 9 of the memorandum, to ensure that these problems do not arise in the future. Owing to the exceptional circumstances which have weakened the financial position of SENELEC and the reasons which led to the use of extraordinary procurement practices, and in view of the remedial actions already taken and planned, the government of Senegal requests a waiver for the nonobservance of these three performance criteria.

4. The government of Senegal believes that the policies and measures set forth in the attached memorandum will permit the achievement of its program objectives; however, it 
will promptly take any additional measures deemed necessary in this connection. The government of Senegal will consult the Managing Director of the IMF-at its own initiative or whenever the Managing Director requests such a consultation - before the adoption of any such measures and in advance of any changes to the policies described in the attached memorandum.

5. To facilitate the attainment of the objectives and the implementation of the abovementioned policies, the government of Senegal hereby requests the completion of the third and fourth reviews as well as the disbursement of the fourth and fifth loans under the current arrangement in an amount equivalent to SDR 13.86 million.

6. On the expiry of the current arrangement on April 27, 2006, the government of Senegal would like to continue its close collaboration with the Fund, through a nonfinancial arrangement, focusing on accelerating growth, reducing poverty and deepening fiscal and financial sector reforms.

7. The government of Senegal consents to the publication of this letter, the memorandum of economic and financial policies, and the report of Fund staff on the third and fourth reviews of the program.

Sincerely yours,

$$
\text { /s/ }
$$

Abdoulaye Diop

Minister of State, Minister of Economy

and Finance

Attachment: Memorandum of Economic and Financial Policies for 2006 of Senegal 


\title{
SENEGAL
}

\section{Memorandum of Economic and Financial Policies for 2006}

\author{
Dakar, December 29, 2005
}

1. In its letter of February 4, 2005 to the Managing Director of the International Monetary Fund (IMF) and in the memorandum attached thereto, the government of Senegal defined its economic and financial objectives for 2005, as well as the measures it intended to implement to achieve them. In this memorandum, the government outlines the results achieved in 2005, its analysis of the deviations observed between projected and actual performance, and the measures it intends to implement in 2006 to achieve its objectives.

\section{MEASURES IMPLEMENTED AND RESULTS ACHIEVED IN 2005}

2. Real GDP growth in 2005 is estimated at 5.1 percent, about 1.3 percent lower than programmed due to the unexpected increase in the price of oil. Growth was driven by recovery of grain production from the decline in 2004 owing to a locust invasion, expansion of construction and public works, and dynamic performance of the telecommunications sector. The consumer price index is estimated to have increased by about 2 percent on an average annual basis, reflecting mainly higher oil prices and the increase in food prices. As a result of the rise in value of petroleum imports, the external current account deficit is expected to increase by about 1 percent of GDP (to 7.6 percent of GDP) rather than narrow (to about 5.5 percent of GDP) as programmed.

3. The overall fiscal deficit in 2005 will remain at 3.5 percent of GDP $(0.2$ percent of GDP more than in 2004), while the basic fiscal surplus will increase slightly (by 0.1 percent of GDP). Strong revenue performance has made it possible to accommodate an increase in the allocation for priority sectors in the supplementary budget, transfers to SENELEC and SAR for the losses incurred as a result of higher oil prices, and the wage bill associated with the finalization of the recruitment program.

4. At end-March 2005, 11 of the 12 performance criteria were hit (Table 1). The continuous performance criterion on the absence of arrears of the electricity company was breached for reasons explained below (paragraph 6). Only half of the six quantitative indicative targets were achieved. The wage bill exceeded the target by CFAF 2.7 billion (less than 0.1 percent of GDP) as delays in recruitment during the last quarter of 2004 were rectified. The expenditures executed under exceptional procedures exceeded the program ceiling, as the treasury experienced difficulties to process the higher than usual volume of payment orders associated with the decentralization of the expenditure process to four ministries. The balance for treasury correspondent accounts was also above the program ceiling, reflecting an accumulation of deposits by some public entities. 
5. At end-September 2005, 2 of the 10 quantitative performance criteria were missed (the continuous ceiling on arrears of the electricity company and the ceiling on no-bid public contracts) for reasons explained below (paragraphs 6 and 9). The same three quantitative indicative targets which were missed in March were again exceeded in September (Table 1). In December 2005, a third performance criterion was missed when the ceiling on the budgetary transfers to SENELEC (continuous PC) was exceeded.

6. The surge in oil prices in 2005 and the reduction by 5 percent and the freezing of electricity prices in July 2004 led to a significant deterioration in SENELEC's financial situation. The company experienced difficulties in meeting obligations toward its suppliers and accumulated arrears on interest charges for overdue payment with one of its supplier (CFAF 1 billion at mid-December 2005). Under current regulation, SENELEC is entitled to budgetary transfers estimated at CFAF 16 billion by the Electricity Sector Regulation Commission (CRSE).

7. In order to improve SENELEC's financial situation, the government first froze the price of the fuel purchased by the company from the refining company (SAR) during JulyOctober 2005, creating the need for a transfer of CFAF 3.8 billion to SAR. In addition, electricity prices will be gradually increased according to a new electricity price formula compatible with the operator's profitability adopted in October 2005. Application of the new formula would entail a 15 percent increase in electricity prices retroactive to January 1,2005 . As a first step, electricity prices were raised by 10 percent on November 1, 2005, and will be further raised in order to fully apply the new price formula by the end of 2006. These adjustments will not be passed on fully to low-income subscribers. If prices are not adjusted in 2006 as required by the formula, CRSE has estimated that the budgetary transfer due to SENELEC would amount to CFAF 37 billion, an amount which could be reduced by about CFAF 9 billion provided two new more efficient power plants come on stream in 2006. SENELEC has signed a memorandum of understanding with its supplier to pay all its arrears before the end of the year.

8. Owing to the exceptional circumstances which have weakened the financial position of SENELEC and in view of the strong remedial actions explained above (paragraph 7), the government requests a waiver for the nonobservance of the performance criteria on the arrears of and the budgetary transfers to SENELEC.

9. The government also requests a waiver for the nonobservance of the ceiling on nobid contracts. This ceiling was exceeded owing to a few large no-bid contracts for the production of digitized identification cards for the next election and military equipment, the strategic nature of which prevented recourse to the usual tender procedures. However, the government recognizes that the excessive use of no-bid contracts is a recurrent problem. In order to limit the recourse to such contracts, the government will implement four remedial actions:

- $\quad$ First, in order to ensure better planning for procurement, each ministry will be required to send procurement plans for 2006 to CNCA (Advisory Commission on 
Public Contracts) by the end of 2005. Ministries that do not comply will be subject to sanctions.

- $\quad$ Second, a revised legal framework for procurement, including the Code of state financial obligations and a procurement code - both prepared with World Bank assistance - will be submitted for parliamentary approval by April 2006. The new framework will strictly limit the conditions under which noncompetitive bids can be approved.

- Third, two new institutions, the Directorate for Public Procurement and the Advisory and Regulatory Authority for Public Procurement, will be set up to enforce the implementation of the revised legal framework.

- $\quad$ Fourth, procurement contracts will be audited every year, with a special emphasis on no-bid contracts. The government will publish these audits, starting in December 2005 with that of the 2003 procurement contracts, and the competent authorities will evaluate the report and propose specific corrective measures, including sanctions.

10. Although two structural benchmarks in the area of fiscal transparency and public expenditure management (PEM) (reports on taxes due and collected and on budget execution) were met with delay, and one structural benchmark (improvement of the transparency of government's table on financial operations) was not observed (Table 2), the government implemented a series of measures that improved tax collection and strengthened PEM. In particular, the government has strengthened the Directorate General of Taxes and Property (DGID) through the recruitment of about 100 staff.

11. To better monitor public finance data, the government has decided to adopt the WAEMU TOFE when the relevant directive under discussion will have been adopted. In the meantime, starting with the TOFE for October 2005, the presentation of the table will be improved as described in the following box (prior action). The provisional monthly tables for January to September 2005 have been produced based on the consolidated monthly treasury balances, with a 60 -day lag. 


\section{Methodological Changes to the Table of Government Financial Operations}

The methodology to report fiscal data will be changed to provide a more accurate assessment of fiscal performance, and a more precise definition of bank and nonbank financing. The new format of the Table of Government Financial Operations (TOFE) brings it closer to the WAEMU TOFE, which expands the coverage of fiscal statistics to local governments and autonomous public entities. The changes are the following:

- Elimination of the line "Treasury Special and CorrespondentAccounts." 17 The accumulation of deposits of Correspondent Accounts at the treasury was previously recorded above the line as negative expenditures. This did not conform to international accounting practices and created distortions. In the new presentation, only those public sector entities whose entire operations (revenue and expenditure) pass through the treasury accounts have been maintained above the line.

- Introduction of the "balance of selected public sector entities" line. This new line in the TOFE, captures the deficit or surplus of three type of institutions: (i) local governments, (ii) autonomous public sector entities (e.g., hospitals, universities), and (iii) the Social Security Fund for civil servants (FNR). Movements in all the other correspondent accounts have been classified as nonbank financing, in accordance with the WAEMU TOFE.

- Revision of the net credit to the government (NCG) series. This series, reported below the line in the TOFE, will be corrected to ensure that its institutional coverage corresponds to the scope of government reflected above the line in the TOFE. This correction will create a difference between the change in the NCG reported in the TOFE and that in the monetary accounts. The latter, reported by the BCEAO, includes public sector agencies whose operations do not have a counterpart "above the line" in the TOFE. The changes in government deposits that do not have a counterpart in the TOFE are listed as a memorandum item. Thus the difference between the TOFE and BCEAO statistics can easily be reconstructed.

${ }^{17}$ Correspondent Accounts are a series of heterogeneous deposit accounts that local governments, autonomous agencies, Social Security Funds, spending ministries, and even private companies and individuals hold at the treasury, which plays the role of a bank. 
12. A number of important steps have been taken to strengthen public expenditure management. Starting with the 2005 budget, a single budget document, containing both current and capital expenditures, was prepared using a harmonized nomenclature. The government issued an administrative order in September 2005, establishing December as a deadline for payment orders (ordonnancements), with exceptions strictly limited to expenditure included in the supplementary budget law.

13. The privatization of SONACOS was completed with the final transfer of assets in March 2005. The specific tax (taxe spécifique) was eliminated in December 2005 (performance criterion). However, in view of the strong increase in vegetable oil imports since 2004, which has weakened SONACOS' financial situation and threatened its ability to buy the groundnut crop, parliament adopted a law imposing import duties of 25 percent on palm oil imports and 15 percent on other vegetable oil imports for a temporary period of up to 6 years. The government commits to ensuring that the imposition of these new taxes is in line with the emergency safeguard clauses in the World Trade Organization (WTO) and WAEMU treaties, within the deadline prescribed by these treaties.

14. The PRSP process has continued to improve health and education outcomes and reduce poverty. According to the second PRSP progress report published in June 2005, the incidence of poverty is estimated to have been further reduced from about 57 percent in 2001 to 54 percent in 2004 . The allocation of spending toward health and education continued to improve, reaching about 33 percent of total spending and 8 percent of GDP in 2004. These increased resources have led to better outcomes. For example, there have been increases in gross enrollment in primary schools from 77 percent in 2003 to 80 percent in 2004, and in the DTC3 immunization coverage from 59 percent in 2003 to 75 percent in 2004.

\section{Government Program for 2006}

15. The macroeconomic outlook for 2006 is broadly favorable. Real GDP growth is projected at about 5 percent, reflecting continued robust expansion in all sectors as in 2005, despite an increase in the average price of oil. Annual average inflation, measured by the GDP deflator, is expected to edge up to about 2.5 percent, reflecting mainly higher oil prices and the increase in electricity prices. Although the rise in value of petroleum imports will weigh on the trade deficit, strong private remittances will help maintain the external current account deficit at about 7.6 percent of GDP.

\section{A. Public Finances}

16. The government's fiscal policy will remain prudent in 2006. The overall fiscal deficit, including grants is expected to increase from 3.5 percent of GDP in 2005 to 4 percent of GDP in 2006, while the basic balance (program definition) will deteriorate by about 1 percent of GDP. This deterioration is explained mainly by higher capital expenditures, the postponement to 2006 of the cost of some structural reforms (especially the recapitalization of the postal agency), and some revenue losses associated with the reduction of the corporate income tax. At the same time, the growth of domestically financed public investment will be 
gradual and will take into account absorptive capacity. In order to improve the efficiency of investment projects, the government will also enhance investment planning, monitoring, and evaluation based on the recommendations of the World Bank's 2005 Public Expenditure Review.

17. The government will maintain an attractive fiscal environment for private sector development. To this end, the corporate income tax will be reduced from 33 percent to 25 percent, effective from January 2006, causing a likely revenue loss of approximately CFAF 19 billion a year. The equalization tax (a special sales tax) will be eliminated in 2007, generating a revenue loss estimated at CFAF 9 billion.

18. The government expects substantial additional revenue from the sale of its minority stake in the telecommunications company SONATEL, the renegotiation of an existing mobile phone license, and the issuance of a new mobile phone license. Senegal's qualification for the MDRI will also free additional resources for priority needs, which will be identified in the new Poverty Reduction Strategy Paper (PRSP) under preparation. The allocation of resources from MDRI will be decided after consultation with Fund staff. A supplementary budget will be presented to parliament in 2006 to account for all resources generated from the telecom sector and debt relief.

19. All resources that may be received within the framework of the Millennium Challenge Account program and the preparation of the summit of the Organization of Islamic Countries are not reflected in the 2006 budget. The amounts and procedures for spending these resources are still not known. In the interest of strengthening fiscal discipline and transparency, the 2006 supplementary budget law will present the external funds made available to the government and all of its agencies, to finance any expenditure associated with these initiatives.

20. To improve the efficiency of tax administration, the government will install a software at the treasury to render the data-sharing system between the three tax collecting agencies fully operational by March 2006. The government will assess the feasibility of transferring direct tax collection responsibilities to the General Directorate for Tax Collection (DGID). The tax management system software SIGTAS will be installed at the large enterprises unit in 2006.

21. The government has prepared an action plan to enhance budgetary transparency (Annex I) in line with the recommendations of the fiscal module of the Report on Observance of Standards and Codes (ROSC), and consistent with the CFAA and CPAR recommendations. The government will strengthen the legal and institutional environment in which various government funds and agencies operate. In particular, it will ensure that agencies follow regular budgetary rules and will set up a system of financial audits for the agencies to enhance transparency regarding financial risks for the government. A comprehensive list of agencies and the budgetary transfers made to them, either directly or through the budget allocations for spending ministries, have been clearly identified in the 2006 budget documentation and will continue to be presented in future budget laws. The 
government will extend the computerized system of monitoring budget execution (SIGFIP) to the final payment phase in 2006, making it possible to produce monthly reports on budget execution by major spending lines until the final payment stage.

22. The government will further strengthen budgetary control procedures. External audits of the government's end-year treasury accounts by the Audit Court will continue, and parliamentary examination of budget review laws will be strengthened. The 2004 end-year treasury accounts and draft budget review laws for 2003-04 will be submitted to the Audit Court by, respectively, end-March and end-June 2006. The capacity of the Audit Court will be enhanced to speed up examination of budget review laws and permit submission to the National Assembly within the timeframe specified by WAEMU directives. Resources allocated to the Audit Court (for current and capital expenditures) will be increased in 200607 to enable it, in particular, to recruit 27 new magistrates.

\section{B. Implementation of the Airport Project}

23. The government has reaffirmed its determination to build the new Blaise Diagne International Airport, the potential benefits of which will be reinforced by the implementation of the toll road and the Diamniadio industrial platform projects. The World Bank has expressed a favorable opinion on certain aspects of the project's viability but has highlighted several important issues that need to be analyzed in depth and resolved before implementation starts. The government commits to finding satisfactory answers to these questions with the World Bank's assistance. Before construction begins, the government will seek the independent and expert opinion of the Council on Infrastructure about the project, and submit it to parliament for its advice. The government will take the results of these deliberations into account in implementing the project.

24. The following measures apply to the implementation of the project:

- $\quad$ An Investment and Operation Company (SIE) will be created, in which the government will hold a 49 percent stake. The government's contribution to the capital of the company will consist of land and will be provided in accordance with company law. The private partner(s) in the airport will hold 51 percent of the capital.

- The company will finance the airport through a loan, which will be reimbursed with the receipts from the Airport Infrastructure Development Tax (RDIA).

- $\quad$ The authorities will publish the annual accounts of the SIE, approved and audited according to international standards, on the external website of the Ministry of Economy and Finance by end-July of the year following the SIE's fiscal year. These accounts will also be forwarded to the parliamentary commission on public works.

- $\quad$ The government will draw up a contract with the SIE, the provisions of which will minimize the risks of the project for public finances. Fund and World Bank staff will receive a copy before the contract is signed, and be given an opportunity to evaluate 
the distribution of risks and financing related to the project. Based on the results of that analysis, the financial flows and the contingent liabilities related to the project will be reflected in the Table of Government Financial Operations (TOFE) and in other fiscal tables as necessary. This contract will set out the procedures governing the use of RDIA revenue for implementation of the project.

- $\quad$ The revenue generated by the RDIA since its introduction on April 1, 2005 will be collected by the International Air Transport Association (IATA). All RDIA revenue will be collected and used in accordance with the procedures defined in Annex II. A decree was adopted to that end (prior action for the third and fourth reviews).

- $\quad$ IATA will be asked to send a report to the Minister of Finance by the end of each month containing full information on: (i) the amount of the funds collected by IATA for the benefit of Senegal directly or through the Administration des Activités Aéronautiques du Sénégal (AAANS) in the previous month and cumulatively since April 1, 2005; (ii) the amount of funds transferred to the SIE and its banks, to the government, or to third parties during the previous month and cumulatively since the first inception of transfers; and (iii) the remaining Senegalese funds held by IATA.

\section{Financial Sector Reform}

25. The government will continue to encourage the development of the financial system and strengthen its supervision. An action plan was prepared for this purpose following broad consultations in 2003 between the government, the private sector, the banking community, and the other financial institutions, and reflecting the recommendations of the Financial Sector Assessment Program (FSAP) report. The priority measures of the plan are presented in Annex III. These measures are intended to, inter alia (i) enhance the soundness of banks, which remains broadly satisfactory, although risk concentration is still the main source of vulnerability in this area; (ii) implement an appropriate legal framework and simplified procedures for the seizure of collateral, by end-December 2005; and (iii) encourage the preparation of certified financial statements by small- and medium-sized enterprises to facilitate their access to credit. The government will take the necessary steps to implement measures under the action plan at the national level, and has already brought those necessitating a decision at the regional level to the attention of the president of the WAEMU's Council of Ministers.

\section{External Debt Policy}

26. The government will not contract or guarantee any foreign loans on nonconcessional terms (as defined in the TMU of February 4, 2005) and will not accumulate any arrears. The provisions regarding prior authorization by the Minister of Finance for any foreign borrowing by public entities, as defined in the November 24, 2003 directive of the Prime Minister, will continue to be strictly enforced. 


\section{E. Public Enterprises}

27. To prevent any adverse consequences on public finances and the economy, the government will continue to strengthen its supervision of the financial situation of public enterprises. The government portfolio management and control unit will be strengthened in order to produce a semiannual report outlining: (i) the level of external and domestic debt of the enterprises in which the government has a stake of at least 45 percent; (ii) the expected new borrowing of these enterprises; (iii) the level of any contingent liabilities arising from the activities of these enterprises; and (iv) the risk to the government of operations related to public/private partnerships. The report on the first half of 2005 will be available in January 2006.

28. Industries Chimiques du Sénégal (ICS), in which the government owns 47 percent of the capital, is in a delicate financial situation and will be monitored closely by the government portfolio management and control unit. In order to resolve its financial problems, private shareholders will recapitalize the company.

\section{F. Accelerated Growth Strategy and Poverty Reduction Strategy Paper}

29. Senegal is committed to achieving the Millennium Development Goals and this objective is clearly reflected in its Poverty Reduction Strategy Paper (PRSP). To that end, the government's growth and development objectives underlying its economic and financial program for 2003-2005 were based on the guidelines specified in the PRSP.

30. To accelerate growth and meet substantial demand for social services, the government has launched deliberations on an Accelerated Growth Strategy (SCA), which aims to increase the average real GDP growth rate to more than 7 percent per annum. In January 2005, a list of five economic activities with substantial growth potential, labor intensity, and external competitiveness was drawn up. These include agriculture and agro-industries, fishing and fishing industries, tourism-art crafts, and cultural industries, textile and clothing industries, and electronic customer support services. In consultation with the public and private sectors, action plans to promote development in these sectors are being prepared for removing constraints to growth. The objective is to start the implementation of the SCA in June 2006.

31. The process of updating the PRSP also began in early 2005 and will be completed by the beginning of 2006. The revised PRSP, with which the SCA action plan will be aligned, will reflect the new priorities, particularly the development of infrastructures, including those in the secondary cities, and enhanced urban mobility in Dakar. It will draw on the conclusions of the first and second PRSP annual progress reports, and emphasize the need to improve the monitoring of poverty reduction indicators, including through better statistics.

\section{RElations With The IMF AND Program Monitoring}

32. The government would like the next economic and financial program supported by the Fund to be a nonfinancial program focusing on accelerating growth, reducing poverty, 
and deepening fiscal and financial sector reforms. Accordingly, the government has established indicative quantitative benchmarks for end-March, June, September, and December 2006 as a basis for assessing performance in the coming months (Tables 3 and 4). In addition, the government will pursue the above-mentioned structural reforms, which could be developed further under the new program to be established on the expiry of the current arrangement on April 27, 2006. 
Table 1. Senegal: Quantitative Performance Criteria and Indicative Targets for 2005

(In billions of CFA francs, cumulative from the beginning of the year, unless otherwise specified; end of period)

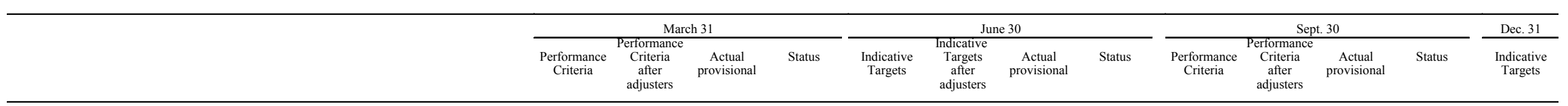

\section{Performance Criteria}

Floor on the basic fiscal balance, excluding temporary costs of structural reforms and spending financed with HIPC-related resources $1 /$ Ceiling on the cumulative change in net bank credit to the government Ceiling on government domestic payments arrears $2 /$

Ceiling on the contrating or guaranteeing of new nonconcessiont

external debt by the government $2 / 3 /$

Ceiling on the amount of government contracts signed without budgetary allocation 2

Ceiling on the share of the value of government contracts signed by single tender (in percent)

Ceiling on

transfers to SONACOS $2 /$

stock of arrears of SONACOS 2

operating losses 2

the stock of arrears of SENELEC 2

SENEL

$\begin{array}{cc}13.5 & \\ -16.1 & 5.2 \\ 0.0 & \\ 0.0 & \\ & \\ 0.0 & \\ 0.0 & \\ 20.0 & \\ & \\ 0.0 & \\ 0.0 & \\ 35.5 & \\ & \\ 0.0 & \\ 0.0 & \\ 52.5 & \\ & \end{array}$

\section{Indicative Targets}

Floor on tax revenue

f current non-wage non-interest expenditure

exceptional procedures

Floor on the difference between the net creditor flow in the treasury accounts of the postal service

and the net creditor flow in the deposit accounts at the Centre de Chèques Postaux (CCP)

and the saving accounts at the Caisse Nationale d'Epargne (CNE) $2 /$

Ceiling on the stock of net deposits in the correspondent accounts of the

treasury, excluding the correspondent accounts of local authorities, pub tc

agencies, SN La Poste, IPRES, and deposit and guarantee accounts

eiling on guarantee deposits of the governmen

$\begin{array}{ccc}26.5 & \text { met } & 32.7 \\ 2.6 & \text { met } & -24.5 \\ 0.0 & \text { met } & 0.0 \\ 0.0 & \text { met } & 0.0 \\ & & \\ 0.0 & \text { met } & 0.0 \\ 0.0 & \text { met } & 0.0 \\ 19.3 & \text { met } & 20.0 \\ & & \\ 0.0 & \text { met } & \text { n.a. } \\ 0.0 & \text { met } & \text { n.a. } \\ \ldots . & \text { n.a 4/ } & \text { n.a. } \\ & & \\ 0.0 & \text { met } & 0.0 \\ 0.9 & \text { not met } & 0.0 \\ 36.6 & \text { met } & 48.4 \\ & & \end{array}$

$\begin{array}{ccc}32.7 & & 1 \\ -24.5 & -24.0 & -8.3 \\ 0.0 & & \\ 0.0 & & \\ & & \\ 0.0 & & \\ 0.0 & & \\ 20.0 & & \\ & & \end{array}$

$\begin{array}{cc}118.3 & \text { met } \\ -81.0 & \text { met } \\ 0.0 & \text { met } \\ 0.0 & \text { met } \\ & \\ 0.0 & \text { met } \\ 0.0 & \text { met } \\ 31.9 & \text { not m } \\ & \text { n.a. } \\ & \text { n.a. } \\ & \text { n.a. }\end{array}$

met
met

$\begin{array}{ccccc}44.0 & & 120.5 & \text { met } & 40.4 \\ -27.3 & -39.6 & -119.4 & \text { met } & -17.5\end{array}$

$\begin{array}{cccc}-27.3 & -39.6 & -119.4 & \mathrm{me} \\ 0.0 & & 0.0 & \mathrm{me} \\ 0.0 & & 0.0 & \end{array}$

Memorandum item

External budgetary assistance, excluding IM

Grants

Loans
Programmed spending of HIPC debt relief

21.0

58.6

18.0

1/ Defined as total revenue minus total expenditure and net lending, excluding externally financed capital expenditure and on-lending, the cost of structural reforms and HIPC spending. 2/ This performance criterion or indicative target will be monitored on a continuous basis.

3/ This criterion excludes government or government-guaranteed CFAF borrowing from financial institutions within WAEMU.

4/ Not applicable: SONACOS was privatized in March 2005.

5/ SENELEC received budgetary transfers amounting to CFAF 16 billion in December 2005. 
Table 2. Senegal: Prior Actions, Structural Performance Criteria, and Benchmarks for the Program under the PRGF Arrangement in 2005

Proposed prior actions for the combined third and fourth reviews

- Promulgate a decree on transparency procedures for financing and construction of Dakar's new airport.

- Improve the transparency of the government's Table of Financial Operations (TOFE) for October 2005 in line with the methodological Changes to the Government Operations Table explained in the box of the memorandum of economic and financial policies in 2006.

\section{Structural performance criteria}

- Submit to the IMF staff the monthly table of the government's financial operations (TOFE) for January 2005.

- Eliminate the specific tax on refined vegetable oil.

- Improve the transparency of the government's Table of Financial Operations (TOFE) in line with the recommendations of the AFR/FAD technical mission of February 2004 concerning the treatment of correspondent accounts and the different government definitions used in the TOFE and the net bank credit to government.

- Prepare a report on commitments, verification, payment orders and payment by major spending lines for the months JanuaryJune 2005, using the software "Système Intégré de Gestion des Finances Publiques" (SIGFIP).

- Implement a new formula for electricity prices compatible with operator profitability.
March 31, 2005

December 31, 2005

March 31, 2005

March 31, 2005

June 30, 2005

Implemented

Implemented

Implemented

Implemented

Implemented with delay

Implemented

Not implemented

July 31, 2005

Implemented with delay

October 1, 2005

Gradual implementation, starting in November 2005 
Table 3. Senegal: Indicative Targets for 2006

(In billions of CFA francs, cumulative from the beginning of the year, unless otherwise specified; end of period)

\begin{tabular}{|c|c|c|c|c|}
\hline & March 31 & June 30 & Sept. 30 & Dec. 31 \\
\hline $\begin{array}{l}\text { Floor on the basic fiscal balance, excluding temporary costs of structural } \\
\text { reforms and spending financed with HIPC-related resources } 1 /\end{array}$ & -3.1 & 3.7 & -13.8 & 0.0 \\
\hline Floor on tax revenue & 208.8 & 464.0 & 669.2 & 892.1 \\
\hline Ceiling on the wage bill & 72.0 & 144.0 & 216.0 & 266.3 \\
\hline Ceiling on the share of the value of government contracts signed by single tender (in percent) & 20.0 & 20.0 & 20.0 & 20.0 \\
\hline $\begin{array}{l}\text { Ceiling on the amount of current non-wage non-interest expenditures } \\
\text { and domestically financed capital expenditures executed through } \\
\text { exceptional procedures }\end{array}$ & 23.0 & 28.1 & 25.6 & 25.7 \\
\hline $\begin{array}{l}\text { Ceiling on the contracting or guaranteeing of new nonconcessional } \\
\text { external debt by the government } 2 / 3 /\end{array}$ & 0.0 & 0.0 & 0.0 & 0.0 \\
\hline
\end{tabular}

1/ Defined as total revenue minus total expenditure and net lending, excluding externally financed capital expenditure and on-lending, the cost of structural reforms and HIPC spending.

2/ This indicative target will be monitored on a continuous basis.

3/ This indicative target excludes government or government-guaranteed CFAF borrowing from financial institutions within WAEMU. 
Table 4. Senegal: Quarterly Government Financial Operations, 2006 1/

\begin{tabular}{|c|c|c|c|c|}
\hline & March & June & Sept. & Dec. \\
\hline & \multicolumn{4}{|c|}{ (In billions of CFA francs, cumulative since the beginning of the year) } \\
\hline Total revenue and grants & 243.2 & 525.6 & 763.6 & 1022.4 \\
\hline Revenue & 217.3 & 480.9 & 701.0 & 934.5 \\
\hline Tax revenue & 208.8 & 464.0 & 669.2 & 892.1 \\
\hline Nontax revenue & 8.5 & 17.0 & 31.8 & 42.4 \\
\hline Grants & 26.0 & 62.7 & 81.4 & 87.9 \\
\hline Budgetary & 9.4 & 9.4 & 9.4 & 16.0 \\
\hline Budgeted development projects & 16.5 & 53.2 & 71.9 & 71.9 \\
\hline Total expenditure and net lending & 269.8 & 631.8 & 930.6 & 1207.3 \\
\hline Current expenditure & 178.9 & 364.7 & 541.7 & 700.7 \\
\hline Wages and salaries & 72.0 & 144.0 & 216.0 & 266.3 \\
\hline Interest due & 9.9 & 24.5 & 32.4 & 46.3 \\
\hline Of which: external & 7.8 & 20.3 & 26.2 & 37.9 \\
\hline Other current expenditure & 97.0 & 196.2 & 293.2 & 388.1 \\
\hline$o / w:$ HIPC current expenditure & 2.2 & 6.5 & 8.6 & 8.6 \\
\hline Capital expenditure & 92.2 & 250.1 & 369.2 & 473.1 \\
\hline Domestically financed & 56.6 & 144.8 & 218.7 & 295.0 \\
\hline Non HIPC financed & 47.7 & 123.0 & 185.7 & 250.5 \\
\hline HIPC financed & 8.9 & 21.8 & 33.0 & 44.5 \\
\hline Externally financed & 35.6 & 105.3 & 150.5 & 178.1 \\
\hline Net lending & -1.3 & 1.5 & 4.3 & 18.0 \\
\hline Temporary costs of structural reforms & 0.0 & 15.5 & 15.5 & 15.5 \\
\hline Selected public sector entities balance $2 /$ & 0.0 & 0.0 & 0.0 & 0.0 \\
\hline Overall fiscal balance (including grants) & -28.6 & -86.0 & -144.1 & -184.9 \\
\hline Overall fiscal balance (excluding grants) & -54.6 & -130.7 & -206.8 & -272.8 \\
\hline Primary balance $3 /$ & -18.8 & -61.5 & -111.7 & -138.6 \\
\hline Basic fiscal balance (program definition) 4/ & -3.1 & 3.7 & -13.8 & 0.0 \\
\hline Financing & 28.6 & 86.0 & 144.1 & 184.9 \\
\hline External financing & 30.7 & 58.7 & 75.3 & 145.5 \\
\hline Drawings & 38.1 & 73.8 & 103.0 & 164.1 \\
\hline Program loans & 16.2 & 16.2 & 16.2 & 31.9 \\
\hline Project loans & 21.8 & 57.5 & 86.8 & 132.2 \\
\hline Amortization due & -12.6 & -38.2 & -54.6 & -132.0 \\
\hline Debt relief and HIPC Initiative assistance & 5.3 & 23.2 & 26.9 & 98.4 \\
\hline T-bills and bonds issued in WAEMU & 0.0 & 0.0 & 0.0 & 15.0 \\
\hline Domestic financing & -2.1 & 27.3 & 68.8 & 39.3 \\
\hline Banking system & -2.1 & 27.3 & 71.6 & 41.0 \\
\hline Of which: T-bills and bonds & 0.0 & 0.0 & 0.0 & 7.5 \\
\hline Nonbank financing & 0.0 & 0.0 & -2.8 & -1.7 \\
\hline Of which : privatization receipts & 0.0 & 0.0 & 0.0 & 1.1 \\
\hline Of which: T-bills and bonds & 0.0 & 0.0 & 0.0 & -2.0 \\
\hline Errors and omissions & 0.0 & 0.0 & 0.0 & 0.0 \\
\hline Financing gap & 0.0 & 0.0 & 0.0 & 0.0 \\
\hline \multicolumn{5}{|l|}{ Memorandum items: } \\
\hline Airport travel tax earmarked for new airport (RDIA) & & & & 16.1 \\
\hline Total HIPC spending & 11.1 & 28.3 & 41.6 & 53.2 \\
\hline Basic fiscal balance (WAEMU definition) 5/ & -14.2 & -40.1 & -70.9 & -68.7 \\
\hline
\end{tabular}

Sources: Senegalese authorities; and staff estimates and projections.

1/ Incorporates methodological changes presented in box of the Memorandum of Economic and Financial Policies.

2/ Local governments, autonomous public sector entities (e.g. hospitals, universities), and the civil servants' pension fund (FNR). 3/ Defined as total revenue and grants minus total expenditure and net lending, excluding interest expenditure.

4/ Defined as total revenue minus total expenditure and net lending, excluding externally financed capital expenditure, on-lending, cost of structural reforms and HIPC expenditure.

5/ Defined as total revenue minus total expenditure and net lending, excluding externally financed capital expenditure, and on-lending.

\section{(CInternational Monetary Fund. Not for Redistribution}




\section{Priority Measures for Improving Fiscal Transparency in 2005-06}

1. Improve the quality of the information presented in the Budget Law, by submitting detailed information on:

(i) all resources transferred to agencies, public entities (établissements publics), funds, 2006 Budget Law and any other budget execution entities either directly or through the budget of spending ministries.

(ii) full details of transfers to public or private enterprises and other public entities

(établissements publics) to compensate them for their quasi-budgetary activities.

2007 Budget Law

(iii) exemptions of direct and indirect taxes, especially those that are associated with the new Investment Code.

2. Prepare the Consolidated Investment Budget (BCI) and the three-year Investment Program using an economic classification of expenditures, starting with the 2007 Budget Law.

3. Shorten the length of the complementary period (periode complémentaire): close out the administrative phase of the expenditure process (payment order or ordonnancement) on December 31, and the accounting phase (payment) at endFebruary of the following year, with exceptions strictly limited to expenditure included in the supplementary budget law.

4. Define the legal framework for agencies, funds, and any other fragmented budget execution entities. Promulgate a law establishing the terms on which agencies and funds may be created, specifying their goals, decision-making bodies, and other mechanisms necessary for tracking and monitoring their operations, and budget execution procedures, including compliance with the Procurement Code.

5. Improve the legal and operational framework for public procurement:

- Forward to the National Commission of Public Procurement (CNCA) the procurement plans for 2006 of all government ministries and agencies.

- Adopt a new Code of State Obligations and Procurement Code that limits the recourse to no-bid contracts.

- Publish the 2003 Audit of Public Procurement and propose corrective

December 2005

December 2005

2007 Budget Law

2007 Budget Law measures, including the need for sanctions.

6. Forward to the Audit Court the end-year treasury accounts (comptes de gestion) for 2004. 


\section{Decree on Transparency Procedures for the International Airport Blaise Diagne de Ndiass}

The following is the text of the articles of the decree that was issued in December 2005 to establish transparency procedures for the use of public resources earmarked for the construction of the new airport.

Article 1. The revenues from the earmarked airport tax (RDIA) will be deposited in a blocked account in a reputable commercial bank of first rank.

The contract with the reputable international commercial bank where the blocked account will be opened includes the following:

(a) The funds deposited in the blocked account can only be withdrawn to service the loan contracted by the Investment and Operation Company for the financing of the airport project (the company).

(b) the depository bank will send a report to the Minister of Finance by the end of each month providing full information on:

- the amounts deposited in the blocked account and interest accrued the previous month and cumulatively since the opening of the account;

- the details of the administrative and banking charges;

- outflows from the blocked account (amounts withdrawn, transferred, paid to third parties, etc.), during the previous month and cumulatively since the opening of the account, by purpose, nature of transaction, and beneficiary; and

- the outstanding balance of the blocked account.

Article 2. The reports provided by the agencies in charge of the collection of the RDIA and by the depository bank will be sent to parliament for information by the Minister of Finance within 15 days of their receipt, and posted on the Ministry of Finance external website.

Article 3. The Minister in charge of the execution and construction of the airport will verify every six months that the use of the proceeds of the loan is consistent with the execution of the works. If the construction work is interrupted, or there are substantial differences between the payments and the progress of the construction work, the Minister responsible for the construction of the airport should ask the company to suspend the withdrawal of funds from the account where the proceeds from the loan have been deposited.

Article 4. The project and the contract between the government and the company will be submitted to the independent Council on Infrastructure and parliament for their advice. The 
statutes of the company and the government's contracts with IATA and the company will be published on the website of the Ministry of Finance.

Article 5. The Minister of State, The Minister of Economy and Finance and the Minister of Tourism and Air Transport are responsible, each according to their areas of expertise, for the implementation of this decree, which will be published in the Official Journal. 


\section{Priority Measures for Enhancing the Performance of the Financial Sector}

\section{Objective}

Improve the legal and judicial environment

Improve prudential regulation

Improve access to credit for SMEs
Boost resources allocated to the judicial system, with the aim of:

Measures

Timetable
- Enhancing the performance of the offices of the clerks of courts;

- Instituting a specialized window to expedite procedures for the realization of collateral;

- $\quad$ Creating specialized chambers within the Dakar courts to handle commercial, financial, and banking cases so as to simplify and expedite procedures for the realization of collateral;

- Increasing the number of judges trained in economic and financial matters and ensuring that court cases are handled regularly throughout the year (including during the judicial holiday period).

Bring the following measures to the attention of the president of the Council of Ministers of the WAEMU:

- Raise the minimum capital adequacy ratio,

- $\quad$ Adopt a law on bank failure in accordance with best international practices.

- Streamline the guarantee funds and the existing interest subsidy mechanisms, as well as their management.

- $\quad$ Provide technical, financial, and institutional assistance (through the Private Investment Promotion Project) to the National Association of Chartered Accountants (ONECCA) to promote the preparation of certified financial statements and prevent the illegal performance of accounting functions.

- $\quad$ Ensure the effectiveness of the ONECCA disciplinary unit.
2006 Budget Law

June 2006

June 2006

June 2006

2006

Implemented, October 2005

December 2006

Ongoing from

September 2005

December 2006 


\section{Senegal: Relations with the Fund}

(As of November 30, 2005)

I. Membership Status: Joined August 31, 1962; Article VIII as of June 1, 1996

II. General Resources Account:

Quota

Fund holdings of currency

Reserve position in the Fund

III. SDR Department:

Net cumulative allocation

Holdings

IV. Outstanding Purchases and Loans:

Poverty Reduction and Growth Facility

(PRGF) arrangements
SDR Million

161.80

160.24

1.56

$\underline{\text { SDR Million }}$

24.46

0.23

$\underline{\text { SDR Million }}$

109.38

Percent of

Allocation

100.00

0.95

Percent of

Quota

67.60

V. Financial Arrangements:

\begin{tabular}{|c|c|c|c|c|}
\hline Type & $\begin{array}{c}\text { Approval } \\
\text { Date }\end{array}$ & $\begin{array}{c}\text { Expiration } \\
\text { Date }\end{array}$ & $\begin{array}{r}\text { Amount } \\
\text { Approved } \\
\text { (SDR million })\end{array}$ & $\begin{array}{r}\text { Amount } \\
\text { Drawn } \\
(\underline{\text { SDR million })})\end{array}$ \\
\hline PRGF & April 28, 2003 & April 27,2 & 24.27 & 10.41 \\
\hline PRGF & April 20, 1998 & April 19, 2 & 107.01 & 96.47 \\
\hline ESAF/PRGF & August 29, 1994 & January 12 & 130.79 & 130.79 \\
\hline
\end{tabular}

\section{Projected Payments to Fund (with Board-approved HIPC Assistance)}

(SDR Million; based on existing use of resources and present holdings of SDRs):

Principal

Charges/interest

Total
Forthcoming

\begin{tabular}{lrrrr}
\hline$\underline{2005}$ & $\underline{2006}$ & $\underline{2007}$ & $\underline{2008}$ & $\underline{2009}$ \\
\hline .41 & 20.96 & 23.75 & 17.86 & 13.20 \\
0.28 & 1.17 & 1.06 & 0.96 & 0.88 \\
4.69 & 22.13 & 24.80 & 18.82 & 14.08
\end{tabular}




\section{Implementation of HIPC Initiative: ${ }^{18}$}

I. Commitment of HIPC Initiative assistance

Decision point date

Assistance committed

By all creditors (US\$ million) $1 /$

Of which: IMF assistance (US\$ million)

(SDR equivalent in millions)

Completion point date

II. Disbursement of IMF assistance (SDR Million)

Assistance disbursed to the member

Interim assistance

Completion point ${ }^{19}$

Additional disbursement of interest income 2/
Enhanced

Framework

June 2000

488.30

42.30

33.80

Apr. 2004

33.80

19.49

4.60

38.40

\section{Safeguards Assessments:}

The Central Bank of the West African States (BCEAO) is the common central bank of eight west African states, which include Senegal. An on-site safeguards assessment of the BCEAO completed on March 3, 2002, proposed specific remedies to alleviate vulnerabilities that were identified by staff. Based on the 2002 financial statements, the staff noted that the BCEAO has improved the explanatory notes to the financial statements and further changes are scheduled for the next fiscal year, with a view towards a gradual alignment with IAS accounting to the extent applicable to central banks by 2005 . The external auditor has apprised the Board of Directors of the BCEAO of the quality of internal controls in June 2003, and the financial statements for the year 2002 were published on the bank's

\footnotetext{
${ }^{18}$ Senegal was not eligible for the HIPC Initiative under the original framework.

${ }^{19}$ Under the enhanced framework, an additional disbursement is made at the completion point corresponding to interest income earned on the amount committed at the decision point but not disbursed during the interim period.
} 
website. The staff will continue its follow up on the progress of the BCEAO in implementing the proposed recommendations as part of the ongoing safeguards monitoring process.

Financial reporting framework. The Fund staff recommended that the BCEAO formally adopt International Accounting Standards (IAS) and publish a complete set of financial statements, including detailed explanatory notes. It was agreed by the BCEAO and Fund staff that the BCEAO will strive to improve its financial and accounting reporting by aligning its practices with those recommended by IAS, which have been adopted internationally by other central banks.

Internal controls system. The staff noted that the absence of oversight of the bank's governance, financial reporting, and internal control practices by an entity external to the management of the BCEAO represented a significant risk. It was agreed y the BCEAO and Fund staff that, after seeking the opinion of the external auditor (Commissaire Contrôleur), the BCEAO staff will propose to the BCEAO Board of Directors that it adopt a resolution whereby the external auditor will be required to apprise the Board of Directors, during its annual review and approval of the financial statements, of the state and quality of internal controls within the bank.

The staff follows up regularly on the BCEAO's progress in implementing the recommendations in the context of the Fund's semiannual regional consultation missions.

\section{Exchange System:}

Senegal is a member of the West African Economic and Monetary Union (WAEMU). The exchange system, common to all members of the union, is free of restrictions on the making of payments and transfers for current international transactions. The union's common currency, the CFA franc, had been pegged to the French franc at the rate of CFAF 1 = F 0.02. Effective January 12, 1994, the CFA franc was devalued and the new parity set at CFAF $1=$ F 0.01. Effective December 31, 1998, the parity was switched to the euro at a rate of CFAF $655.96=€ 1$. On May 6, 2005, the rate of the CFA franc in terms of the SDR was SDR $1=$ CFAF 767.75 .

Aspects of the exchange system are also discussed in the recent report on economic developments and regional policy issues of WAEMU.

\section{Article IV Consultations:}

Senegal is on the 24-month Article IV consultation cycle, in accordance with the provisions of the decision on consultation cycles. The 2004 Article IV consultation was completed by the Executive Board on March 7, 2005 (2004 Article IV consultation and the second review, and IMF Country Report No. 05/155). In concluding the Article IV consultation, Executive Directors stressed the importance of prudent fiscal policies and structural reforms to enhance policy credibility, maintain debt sustainability, and achieve the growth needed to further 
reduce poverty. They emphasized in particular the need to enhance public expenditure management and fiscal transparency, rehabilitate and privatize public enterprises, address the under-development of financial markets, and press ahead with structural reforms and infrastructural improvements to lower the cost of production, improve the business climate and raise productivity in the export sector.

\section{Financial Sector Assessment Program (FSAP) and Report on the Observance of Standards and Codes (ROSC) Participation:}

A joint team of the World Bank and the International Monetary Fund conducted a mission under the FSAP program in November 2000 and January 2001. The Financial System Stability Assessment (FSSA) was issued in August 2001 (IMF Country Report No. 01/189). An FSAP update was undertaken in June 2004, focusing on development issues (in particular nationwide supply of basic financial services and access of SMEs to credit), in line with the priorities defined in the PRSP (IMF Country Report No. 05/126).

A ROSC on the data module, based on a September 2001 mission, was published on December 2, 2002. An FAD mission conducted a ROSC on the fiscal transparency module in January 2005.

\section{Technical Assistance:}

\begin{tabular}{|c|c|c|c|}
\hline STA & Staff & September 2001 & ROSC assessment of data. \\
\hline FAD & Staff/consultant & September 2001 & $\begin{array}{l}\text { Assessment of capacity to track } \\
\text { poverty-reducing expenditures. }\end{array}$ \\
\hline STA & AFRISTAT & July 2002 & $\begin{array}{l}\text { Real sector statistics assessment } \\
\text { Mission, under GDDS West } \\
\text { Africa project. }\end{array}$ \\
\hline STA & AFRISTAT & August 2002 & $\begin{array}{l}\text { National accounts assistance } \\
\text { under GDDS West Africa } \\
\text { project. }\end{array}$ \\
\hline STA & Regional advisor & August 2002 & $\begin{array}{l}\text { Continued assistance with fiscal } \\
\text { sector data under GDDS West } \\
\text { Africa project. }\end{array}$ \\
\hline STA & AFRISTAT & December 2002 & $\begin{array}{l}\text { Continued assistance with } \\
\text { national accounts and prices } \\
\text { statistics under GDDS West } \\
\text { Africa project }\end{array}$ \\
\hline
\end{tabular}


STA Regional advisor February $2003 \quad$ Continued assistance with fiscal sector data under GDDS West Africa project.

AFRITAC 2003

- Ongoing

Public external debt: Upgrading of information systems; techniques of external debt management.

AFRITAC

November 2003

- Ongoing

Assistance to strengthening the microfinance sector.

\section{Resident Representative}

Stationed in Dakar since July 24, 1984. The position has been held by Mr. Ousmane Doré since August 4, 2003.

\section{Fourth Amendment of the Articles of Agreement and the Eleventh Quota Review}

The authorities have indicated their agreement with the Fourth Amendment of the Articles of Agreement. The increase in Senegal's quota under the Eleventh General Review of Quotas was completed on February 11, 1999. 


\section{Senegal: Relations with the World Bank ${ }^{20}$}

(As of December 21, 2005)

\section{Partnership in Senegal's development}

1. In May 2002, the Government of Senegal outlined its development strategy in a poverty reduction strategy paper (PRSP). The PRSP was presented to the Bank and IMF Boards in December 2002. It covers the period 2003-2005. The Government provided the first annual PRSP progress report in March 2004 and the second Annual Progress Report in June 2005. The PRSP sets out the following pillars of the government's strategy: (i) creation of wealth within a healthy macroeconomic framework; (ii) capacity building and promotion of basic social services; (iii) improving the living conditions of vulnerable groups; and (iv) implementation and monitoring/evaluation. A new PRSP is currently being prepared and the Government expects to finalize it by first half of 2006.

2. Regarding the division of responsibilities between the Bretton Woods institutions, the IMF takes the lead in the policy dialogue on macroeconomic policies and monitors macroeconomic performance by way of quantitative performance criteria and indicators. In addition, the IMF's PRGF contains structural conditionality in areas such as electricity and groundnut sector reform, which have a direct bearing on macroeconomic stability and growth prospects.

3. The PRSP and its comprehensive poverty analysis have provided the framework for the Bank's country assistance strategy (FY03-05). The Bank supports the Government's efforts to achieve sustained growth rates, reduce the incidence of poverty and improve access to basic social services. In particular, the Bank is currently supporting the implementation of the PRSP in the areas of health/nutrition, education, HIV/AIDS, rural development, transport, water, and urban development through the implementation of a portfolio of specific projects, as outlined more fully below. A new CAS (FY07-10) will be prepared to take into account the Government's priorities as set in its new PRSP. CAS Board presentation is currently planned for December 2006.

\section{World Bank Group strategy and portfolio}

\section{Lending}

4. IDA has provided external assistance to Senegal since 1966. The main objective of the Bank's assistance strategy for Senegal has been to reduce the incidence of poverty and improve employment. The Bank is working to (i) develop country ownership through policy dialogue;

(ii) use public expenditure reviews with a focus on impacts at the levels of the consolidated central budget; (iii) monitor linkages between implementation and aggregate results; and (iv) emphasize investment in human capital through the lending and advisory services.

${ }^{20}$ Prepared by Françoise Perrot from the World Bank (202-473 4465). 
5. As of December 21, 2005, the World Bank had approved 132 credits for Senegal with a total amount of about US\$2.6 billion. Past projects had supported agricultural diversification, irrigation, human resources development, institutional development, and expansion of the country's infrastructure, particularly its transport system. In recent years, the emphasis has shifted to better utilizing and maintaining existing facilities and to helping the Government resolve some of the key issues hampering long-term development prospects. The current active portfolio has a commitment value of about US\$672.2 million equivalent, with an undisbursed amount of about US\$402.6 million. The portfolio is composed of 17 credits in various sectors (rural development, human resources: population/health/nutrition/education/social development, HIV/AIDS, infrastructure/urban development, energy/water, industry, and private sector development, budget support).

\section{Sector issues}

6. Bank support to the health sector is provided under a series of PRSCs. PRSC II is expected to strengthen the Government's efforts aimed at improving health infrastructure and the allocation of human and financial resources toward the regions.

7. In 1999, the Government adopted a ten-year program (PDEF) with the ultimate objective of reaching universal primary education by the year 2008 (up from 60 percent of gross primary enrollment in 1999). A Quality Education For All Project (QEFA-Education SIP) covers the first three years of the ten-year program. Key issues in education include the need to (a) consolidate gains in expanding access while addressing the needs of the under- and un-served areas; (b) support quality improvements in education through interventions at the school and at local education structures; (c) improve sector management (in particular, financial and personnel management); and (d) prepare for smooth deconcentration, and eventual decentralization, to the regional and departmental levels. The first phase of the QEFA operation is due to close at the end of December 2005. Preparation of the second phase is under way. Board presentation is planned for the second half of FY06.

8. Up until 1996, the urban water sector was facing two major issues: (i) a shortage of water production and distribution capacity in the Dakar area requiring substantial immediate investments, (ii) low managerial efficiency with no financial viability of the sector. To deal with these issues, the government adopted two Water Sector Projects in 1996 and 2001 supported by seven donors, including the World Bank as lead agency. The Water Sector is now closed. The Long-Term Water Sector project includes a large physical investment program and institutional reforms (PSP), which have increased access to potable water in the Dakar area and improved overall management of the sector.

9. In the transport sector, capacity constraints are being addressed with a program of new investments, especially in rehabilitating rail links between Senegal and Mali, as well as institutional reforms. The Transport SIP was launched in 1999 with support from several donors, including the World Bank. Key issues in transport include the need to strengthen institutional capacity, direct private sector involvement in investment and management of the sector, improving the condition of the priority road network and reducing the cost of road maintenance and rehabilitation. 
10. Due to global difficulties in the energy sector, the two "privatization" efforts conducted over the last five years to bring private sector expertise and private financing into SENELEC were not successful. The Bank is now assisting the Government in a third effort taking into account the lessons learned and the overall international context where private sector interest in investing in Africa is reduced. A Rural Electrification Services project was approved in September 2004, and an Electricity Efficiency Enhancement project was approved in May 2005.

11. As of September 2005, credits from the International Finance Corporation's (IFC) portfolio totaled about US\$38 million and included two targe investments (the GTI-Dakar power plant - the first independent power producer of the country - and Ciments du Sahel - the country's second cement producer). Direct SME investments were made to finance a 130-room hotel, a private school, a microfinance institution, and expand a fishing fleet. Prior equity investments in the financial sector in housing and leasing are mature and IFC is seeking exit. IFC has been active in advisory work with FIAS assessments of investment red tape and the taxation system. IFC is also an active participant in the President's Investors' Advisory Council, a forum for high-level dialogue between the government and private sector (domestic and foreign).

\section{World Bank-IMF collaboration in specific areas}

12. The IMF and the World Bank staff maintain a close working relationship, especially with respect to (i) the implementation of the Poverty Reduction Strategy; (ii) reforms in public finance management; and (iii) structural measures in specific sectors, such as electricity and groundnuts, which have systematic impact on the public finances and on macroeconomic stability.

13. The conditionality for the groundnut and electricity sectors in the new PRGF-supported program has been developed in close collaboration with World Bank staff, and the Bank takes the lead role in working out the technical details of the envisaged reforms. The IMF and the Bank also coordinate their activities and conditionalities in the area of public expenditure reform, an area in which both institutions have an interest. 
Senegal: Statement of Loans/Credits/Grants

(As of July 26, 2005)

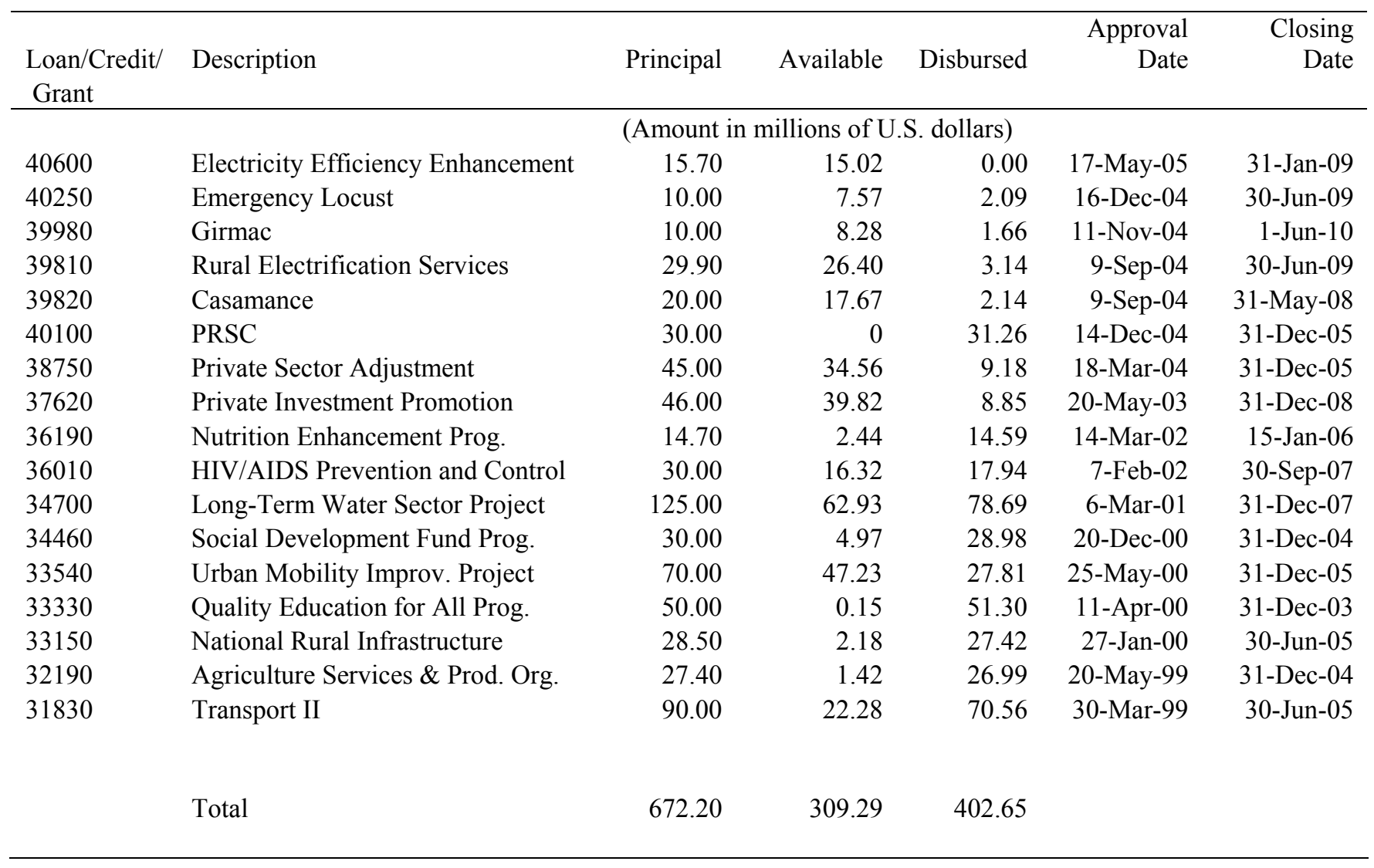


Statement of IFC's Held and Disbursed Portfolio

(As at 9/20/05)

(In US\$ million)

\begin{tabular}{|c|c|c|c|c|c|c|c|c|c|}
\hline \multirow[b]{2}{*}{ FY Approval } & \multirow[b]{2}{*}{ Company } & \multicolumn{4}{|c|}{ Held } & \multicolumn{4}{|c|}{ Disbursed } \\
\hline & & Loan & Equity & Quasi & Partic & Loan & Equity & Quasi & Partic \\
\hline $1996 / 97$ & AEF SERT & 0 & 0.43 & 0 & 0 & 0 & 0.43 & 0 & 0 \\
\hline 1980 & BHS & 0 & 0.46 & 0 & 0 & 0 & 0.46 & 0 & 0 \\
\hline 1999 & $\mathrm{CDS}$ & 14.59 & 2.26 & 3.25 & 0 & 14.59 & 2.26 & 3.25 & 0 \\
\hline $1997 / 98$ & GTI Dakar & 9.06 & 1.69 & 0 & 10.93 & 9.06 & 1.51 & 0 & 10.93 \\
\hline \multirow[t]{2}{*}{2001} & SEF Royal Saly & 1.48 & 0 & 0 & 0 & 1.48 & 0 & 0 & 0 \\
\hline & Portfolio: & 25.13 & 4.84 & 3.25 & 10.930 & 25.13 & 4.67 & 3.25 & 10.93 \\
\hline
\end{tabular}

AEF SERT = Africa Enterprise Fund - Société d'exploitation des ressources thonnères (tuna fishing unit) BHS = Banque Habitat du Sénégal (local housing bank)

CDS = Ciments du Sahel (cement factory)

GTI-DAKAR $=$ (power plant)

SEF Royal Saly $=$ Small Enterprise Fund (small tourist hotel) 


\section{Senegal: Statistical Issues}

9. Overall, Senegal's economic database is comprehensive, but there are weaknesses in data on national accounts, production, international trade, and social indicators. The authorities are strongly committed to improving the quality and availability of economic, financial and social indicators, partially relying on technical assistance from the Fund and other international organizations.

10. Senegal has embarked on a process of regional harmonization of statistical methodologies within the framework of the West African Economic and Monetary Union (WAEMU).

11. Senegal participates in the General Data Dissemination System (GDDS), and its metadata were posted on the Fund's Dissemination Standards Bulletin Board on September 10, 2001. Metadata were updated in July 2005.

12. The mission that prepared the Report on Observance of Standards and Codes (ROSC) in September 2001 carried out a review of Senegal's data dissemination practices against the GDDS, as well as an in-depth assessment of the quality of national accounts, consumer price, monetary, balance of payments, government finance, and income poverty statistics. The main findings of the mission were as follows: (i) Senegal generally follows the recommendations of the GDDS for the coverage, periodicity, and timeliness of all data categories; (ii) improvements need to be made in data coverage (especially national accounts by institutional sectors), periodicity (especially quarterly government financial statistics), and timeliness (especially balance of payments, monetary, and poverty); and (iii) plans for improvement in these areas should address resource constraints, reinforce the legal framework for data collection and coordination, and clarify the responsibilities for dissemination of government operations, public debt, and monetary data. The mission also found that, while statistics were generally compiled based on acceptable sources; those relating to government finance were weak because of the absence of an integrated accounting source and the practice of not investigating revisions.

13. On October 17, 2002, the authorities conveyed to the Fund their observations on the draft data ROSC and their consent to the publication of the final version on the Fund website. The data ROSC was published on December 2, 2002.

14. In discussions with the government in the context of the 2002 Article IV consultations and the monitoring of the PRGF-supported program, the staff has urged the authorities to address the lack of quarterly (or half-yearly) indicators for overall economic activity. The national accounts and balance of payments data are prepared only on an annual basis. The authorities publish a quarterly industrial production index and a semi-annual services sector output index.

15. Specific issues in various topical areas are discussed below. 


\section{Real sector}

16. The comparability of the national accounts generally follows the System of National Accounts, 1993. While the staff demonstrates professionalism, the lack of adequate financial resources has constrained efforts to collect and process data. Data sources are deficient in some areas, particularly the informal sector. Owing to financial constraints, surveys of business and households are not conducted regularly, impeding the production of national accounts estimates (e.g., input-output tables and institutional sector accounts are not compiled annually). However, efforts are being made to improve data collection procedures, strengthen the coordination among statistical agencies, and reduce delays in data dissemination. A project to implement the System of National Accounts 1993 was completed in early 2003 and included a change in the base year from 1987 to 1999, and improvement of estimates of informal activities; revised data have already been published in the IFS. Other initiatives, as indicated in the February 2004-April 2005 AFRITAC West Work Program, are the redevelopment of the industrial production index, development of a statistical register, production of a CPI with national coverage. A regional advisor in real sector statistics covering the West Afritac countries, including Senegal, was posted for one year beginning December 2005.

17. The coverage of the harmonized consumer price index, introduced in January 1998, is limited to Dakar. Its weights are based on a household budget survey conducted during only three months in 1996, and the regular provision of financial resources required for the price collection is not assured.

\section{Public finances}

18. Government finance statistics (GFS) data are compiled by the Ministry of Economy and Finance from the customs, tax, and treasury directorate sources. The authorities have not reported data to STA for inclusion in the 2005 GFS Yearbook. Data last reported were for fiscal year 2001. They do not report subannual data for publication in IFS, although the ministry compiles and disseminates reasonably detailed quarterly government financial operations tables (TOFE) in their own publications. In part to improve its GFS, Senegal started implementing recommendations made by a May 2000 FAD mission to correct a reported deterioration in the treasury accounts, as well as to integrate special accounts.

19. An AFR team worked with the authorities in February 2004 to improve fiscal reporting in the context of the PRGF-supported program. The focus was on (i) public accounts that are outside of the direct purview of the treasury; (ii) the treatment of correspondents' accounts in the TOFE; and (iii) ensuring consistency between treasury and banking system information concerning government transactions. The mission found that the recent adoption by the authorities of the five WAEMU directives on public finances had set off a series of reforms, which should contribute to improving the overall government financial operations and bring the TOFE much in line with the extended WAEMU TOFE. However, the full implementation of these reforms could take time because of delays in addressing the weakness in the treasury computerization system. In the interim, the mission proposed a number of specific recommendations for improvement of the fiscal accounting 
practices. The authorities will review with Fund staff the modalities for incorporating these recommendations.

\section{Monetary data}

20. Preliminary monetary data for Senegal are compiled by the national agency of the Central Bank of West African States (BCEAO) and officially released by BCEAO headquarters. There has been an improvement in the timeliness of data provided on interest rates, monetary institutions, and deposit money banks. The authorities are now reporting monetary data to STA on a regular basis, with a reduction in the lag from about six months to about three to four months. Most key monetary statistical issues have been resolved.

21. A monetary and financial statistics mission visited BCEAO headquarters in Dakar in May 2001. The mission reviewed the procedures for collecting and compiling monetary statistics and addressed outstanding methodological issues that concern all the member countries of the WAEMU. The mission also briefed the BCEAO authorities on the methodology in the new Monetary and Financial Statistics Manual (MFSM) and discussed the modalities for introducing an IFS area-wide page for the WAEMU zone, which was subsequently introduced in the January 2003 issue of IFS.

22. In April 2003, the BCEAO organized a regional seminar on monetary and financial statistics with representatives from BCEAO headquarters and national agencies in Dakar; STA staff also participated. Participants agreed to set up a working group consisting of representatives from the national agencies and various departments of the BCEAO's headquarters, which will follow up on the implementation of the seminar's recommendations on implementation of the MFSM.

\section{Balance of payments data}

23. Balance of payments data for Senegal are compiled by the national agency of the BCEAO. With STA support, several steps have been taken to tackle balance of payments deficiencies, including: (i) implementation of the Balance of Payments Manual $5^{\text {th }}$ edition methodology; (ii) modified and simplified related surveys for companies and banks; (iii) improvement in the computerization of procedures; and (iv) significant strengthening of staff training. Definitive balance of payments data can now be provided with a delay of less than one year. Further efforts are required to enhance the quality and coverage of balance of payments data. In particular, their latest published data show significant inconsistencies between the balance of payments and the international investment position. 
Senegal: Table of Common Indicators Required for Surveillance

(As of December 20, 2005)

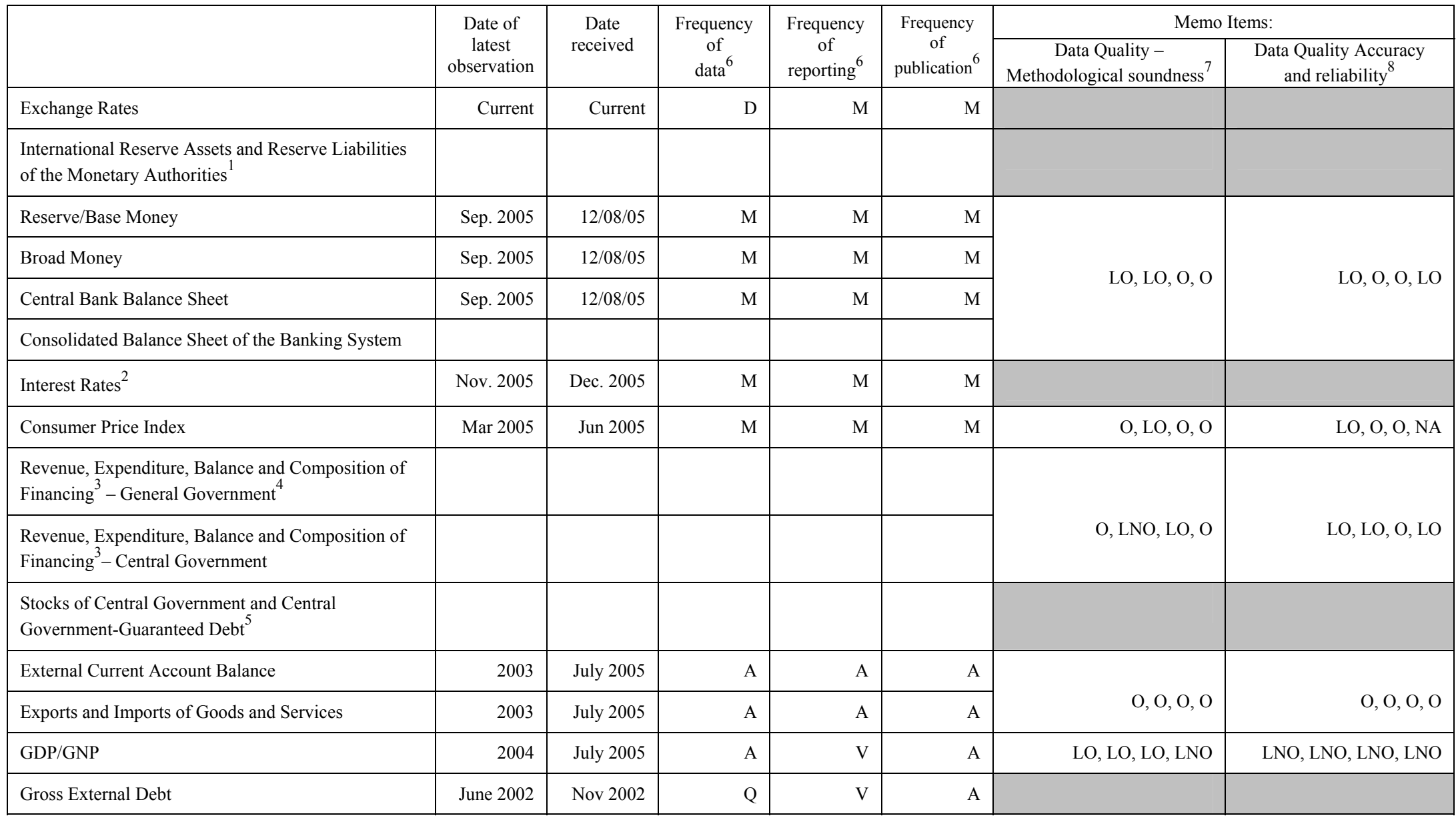

${ }^{1}$ Includes reserve assets pledged or otherwise encumbered as well as net derivative positions.

${ }^{2}$ Both market-based and officially-determined, including discount rates, money market rates, rates on treasury bills, notes and bonds.

${ }^{3}$ Foreign, domestic bank, and domestic nonbank financing.

${ }^{4}$ The general government consists of the central government (budgetary funds, extra budgetary funds, and social security funds) and state and local governments.

${ }^{5}$ Including currency and maturity composition.

${ }^{6}$ Daily (D); Weekly (W); Monthly (M); Quarterly (Q); Annually (A); Irregular (I); Not Available (NA).

${ }^{7}$ Reflects the assessment provided in the data ROSC published in November 2002 and based on the findings of the mission that took place in September 2001 for the dataset corresponding

to the variable in each row. The assessment indicates whether international standards concerning (respectively) concepts and definitions, scope, classification/sectorization, and basis for

recording are fully observed (O), largely observed (LO), largely not observed (LNO), not observed (NO), or not available (NA).

${ }^{8}$ Same as footnote 7, except referring to international standards concerning (respectively) source data, statistical techniques, assessment and validation of source data, and revision studies. 
January 10, 2006

\section{IMF Executive Board Completes Third and Fourth Reviews Under Senegal's PRGF Arrangement and Approves US\$20.0 Million Disbursement}

The Executive Board of the International Monetary Fund (IMF) has completed the third and fourth reviews of Senegal's economic performance under an SDR 24.3 million (about US\$35.1 million) Poverty Reduction and Growth Facility (PRGF) arrangement. The three-year PRGF arrangement was approved on April 28, 2003(see Press Release No. 03/62). The completion of the reviews enables a further release of an amount equivalent to SDR 13.9 million (about US\$20.0 million) The disbursement brings Senegal to the maximum amount available under the arrangement. In completing the reviews, the Board also granted a waiver for the nonobservance of performance criteria.

Senegal was one of 19 countries recently granted 100 percent relief on all obligations to the IMF incurred before January 1, 2005 (see Press Release No. 05/302). Total debt relief granted to Senegal amounts to approximately US\$144.9 million, including relief under the Heavily Indebted Poor Countries Initiative. Excluding HIPC relief, the relief amounts to US\$136.9 million and will be effective in early January 2006.

Following the Board discussion of Senegal, on January 9, 2006, Mr. Agustín Carstens, Deputy Managing Director and Acting Chair, stated:

"The Senegalese authorities are to be commended for maintaining robust economic growth and low inflation in 2005 despite higher world oil prices. The fiscal deficit and government indebtedness remain at sustainable levels, and progress in structural reforms has continued. Going forward, full implementation of the reform agenda will be critical to sustaining donor support and achieving the Millennium Development Goals.

"The authorities are strongly committed to implementing their program of economic and financial policies geared toward growth and poverty reduction. The fiscal program for 2006 allows for a modest increase in expenditure, keeps the investment outlays in line with the absorptive capacity of the economy and foreign financing, and aims at a realistic revenue target. The authorities should allocate more resources to the health and social service sectors in the 2006 supplementary budget, taking advantage of the resources made available through the Multilateral Debt Relief Initiative. 
"Progress in strengthening public expenditure management and enhancing fiscal transparency and governance is crucial to raise the efficiency of public outlays and the economy's growth potential. In particular, more needs to be done to improve investment planning, evaluation, and monitoring further; fully implement transparent procedures introduced for the construction of the new airport; and strictly enforce the new procurement code.

"Sustained economic growth and poverty reduction will also require further progress in structural reforms, particularly in the public enterprise sector. To rehabilitate the chemical company, ICS, the government should press for cost cutting, management improvements, and capital injection by private partners. The financial situation of the state electricity company, SENELEC, should be strengthened, by adhering to the market-oriented electricity formula for setting electricity prices, with safeguards to protect poor households. The authorities should consider rescinding the new regressive tax aimed at protecting the recently privatized groundnut processing company, SONACOS, from foreign competition.

"Efforts should continue to enhance the soundness of the banking sector, and priority actions identified in the action plan should be taken expeditiously. This will also require strengthened coordination at the regional level," Mr. Carstens said.

The PRGF is the IMF's concessional facility for low-income countries. PRGF-supported programs are based on country-owned poverty reduction strategies adopted in a participatory process involving civil society and development partners, and articulated in a Poverty Reduction Strategy Paper, or PRSP. This is intended to ensure that each PRGF-supported program is consistent with a comprehensive framework for macroeconomic, structural, and social policies, to foster growth and reduce poverty. PRGF loans carry an annual interest rate of 0.5 percent, and are repayable over 10 years with a 51/2-year grace period on principal payments. 


\section{Statement by Damian Ondo Mañe, Executive Director for Senegal January 9, 2006}

1. We thank staff for the comprehensive set of papers on Senegal and for providing the authorities with useful policy advice. We also would like to commend Management and staff for their diligence in bringing these papers within tight deadlines for Board consideration. We express gratitude to Directors for agreeing to waive the minimum circulation period in this case. We are pleased to convey to the Executive Board the authorities' appreciation of its decisive role in Senegal's qualification under the MDRI debt relief. The decision that Senegal meets all the criteria to qualify for immediate debt relief is a clear recognition of the authorities' sound macroeconomic policies, consistent implementation of the PRS, and robust PEM systems. Nevertheless, my authorities view the MDRI debt relief as an invitation to persevere in strengthening macroeconomic and structural policies, enhancing budget management and their poverty reduction strategy with a view to achieving growth and poverty reduction objectives and making the best use of the resources freed by the multilateral debt relief to that effect.

\section{SENELEC ANd Misreporting Allegations}

2. As noted by staff, the financial situation of the electricity company, SENELEC, worsened last year in the aftermath of oil price increases. An action plan has been already implemented with a view to strengthening the financial situation of the company. Key measures include inter alia (i) a 10 percent increase in electricity tariffs on November 1 , 2005 which is a first step toward ensuring a full pass-through of oil price increases by end2006, and (ii) adjustment measures taken by SENELEC to improve its profitability. In view of these measures which should improve the financial situation of SENELEC, the authorities request a waiver for the nonobservance of the performance criteria on the arrears of and budgetary transfers to the company.

3. With regard to the noncomplying disbursement, we wish to echo the authorities' view that "there was no intent to withhold information nor did misreporting take place, and certainly there was no deliberate intent to provide the Executive Board with inaccurate information concerning SENELEC's late interest due to the independent electricity producer." We would like to point out that there was a tacit agreement between SENELEC and its energy supplier, GTI, which granted the former a deferral of payment, at least until the latter claims payment of such interest. In view of the terms of this agreement, it cannot be ascertained that there were any arrears on interest charges on payment to GTI at least so long as GTI did not make a demand for payment of late interest. Moreover, at the time GTI demanded payment of late interest in June 2005, there was disagreement as to the exact amount of interest due, which made it difficult to estimate the arrears of SENELEC. Upon reaching an agreement with GTI on the amount of the late interest, a plan to clear the arrears was agreed upon and SENELEC began to repay promptly on December 15, 2005. Given these circumstances, it is the authorities' view that there was neither accumulation of arrears nor misreporting to the Fund. 


\section{Recent Macroeconomic and Structural DeVelopments}

4. Macroeconomic performance has been strong under the PRGF arrangement, with GDP growth rates exceeding 6 percent in 2003 and 2004. For 2005, preliminary estimates indicate that, in the absence of the oil price shock, GDP growth estimated at 5.1 percent could have been along the lines of growth rates reported during the two previous years. Owing to this shock, inflation bounced but stayed within the program limits and the external current account deficit increased by slightly less than one percentage point of GDP.

5. On the fiscal front, strong revenue performance was made possible last year, in part, by the authorities' efforts to enhance collection of corporate income tax revenues. Total expenditure increased during the same year, accommodating the much-needed infrastructure investment and unexpected spending related to the oil price increase, including transfers to the electricity company and oil refinery.

6. Overall, public expenditure management has been in line with the poverty reduction and growth objectives that the authorities strive to achieve through the implementation of the PRGF-supported program. This is notably reflected by the increasing trend in public investment and social expenditure. Actions taken to strengthen budget management include the decision made by the government last September to halt payment orders each December except for expenditure set forth in the supplementary budget law. The authorities are mindful of the staff concern about the excessive use of no-bid contracts. They are determined to take immediate actions with a view to strengthening procurement practices, including enhanced procurement planning, the submission for parliamentary approval of a revised legal framework for procurement practices, the creation of new agencies to ensure the implementation of the revised legal framework, and the yearly audit of procurement contracts. In light of the above, the authorities request a waiver for the nonobservance of the performance criteria on no-bid contracts.

7. On the structural front, delays in implementing the benchmark on the revised presentation of the main fiscal table - converted into a prior action-were related to the process of program negotiations. As agreement was finally reached with staff on the necessary methodological changes to this table, the authorities moved promptly toward the implementation of the related structural condition.

8. Furthermore, it is important to note that the authorities' strategy to cope with the adverse impact of the increased imports of vegetable oil and address the difficult financial situation of the groundnut processing and vegetable oil refining company, SONACOS, was developed in close collaboration with the World Bank and in accordance with the WTO's Agreement on Safeguards. Measures taken to that effect were, among other things, motivated by the authorities' desire to protect the livelihood of the many small farmers involved in the groundnut sector whose income would otherwise be adversely affected, thereby aggravating the incidence and depth of poverty. 


\section{Prospects AND Challenges fOR 2006}

9. The outlook for 2006 remains encouraging, with GDP growth and the external current account deficit projected to be at the same level as in 2005 and CPI inflation expected to rise to 2.5 percent before trending downward in subsequent years.

\section{Fiscal Policy and Reform}

10. The fiscal outlook for 2006 is consistent with macroeconomic stability. Still, the authorities intend to limit increases in public investment, in order to allow at most an increase of one-half percentage point of GDP in overall fiscal deficit-from 3.5 percent of GDP in 2005 to 4 percent of GDP in 2006 - while paying due attention to absorptive capacity constraints. With the World Bank's assistance, care will be also taken to increase the productivity of infrastructure investment through better planning, monitoring, and evaluation.

11. With regard to tax revenue, the authorities intend to install a tax management system software during the course of this year. Actions are also envisaged in the coming months to enhance the connection between the Treasury and the tax collecting agencies through the establishment of a data-sharing system. The reduction in the corporate income tax effective as of January 2006 reflects the authorities' desire to boost private sector development and is expected to have a minor incidence on revenue performance.

12. Enhancing fiscal transparency is among the main objectives pursued by the authorities as well as a key pillar of their macroeconomic program. To that end, an action plan was designed by the government based on the recommendations of the fiscal module of the ROSC, and close collaboration was maintained with the World Bank through the implementation of Country Financial Assessment Accounting and Country Procurement Assessment Review action plans.

\section{Airport Project}

13. The airport project is a manifestation of the authorities' determination to explore innovative ways of financing major infrastructure projects deemed necessary for the development of the economy. A delegation headed by the Prime Minister made a visit to the Fund in June 2005 during which a detailed presentation of the airport project was made to the staff. The authorities explained that the financing scheme of the airport project was in full compliance with the regulations of the International Civil Aviation Organization.

14. From the very beginning, the authorities shared the staff's call for the need to ensure full transparency in the implementation of the project. However, differences on the nature of transparency procedures to be implemented led to lengthy discussions that finally came to a successful conclusion last month. The authorities have already started to implement the details of the agreement reached with staff on the transparency procedures including the promulgation of a decree on transparency procedures related to the financing and construction of the airport. 


\section{Financial Sector Reform and Public Enterprises}

15. Although the soundness of the banking sector corroborated by the last FSAP mission is still maintained, the authorities continued to work on reducing vulnerabilities related to banks' exposure to large corporations. After extensive consultation with domestic stakeholders, the authorities have finalized an action plan aimed at strengthening the financial system and enhancing its supervision. This plan based on the FSAP recommendations envisages a series of measures to reduce nonperforming loans and the vulnerability in the financial sector induced by credit concentration. The main objectives of the priority measures of the action plan are to strengthen the legal and judicial framework, improve prudential regulation, and provide enhanced access to credit for small and medium enterprises. Specific measures to improve prudential regulation were already brought to the competent regional authorities, including the increase in the minimum capital adequacy ratio and the adoption of a law on bank failure in line with best international practices.

16. Concerning the Industries Chimiques du Sénégal, the government has agreed to an increase in the capital of this company with a view to allowing the private partner to recapitalize the company and detain the majority stake.

\section{Accelerated Growth Strategy (SCA) and Poverty Reduction}

17. As we noted during the last Board meeting on Senegal, the government of Senegal has decided to place a special emphasis on an accelerated growth strategy (SCA) in order to reach the objective of halving the poverty rate by 2015 and create export opportunities. The SCA was launched early in 2005 after broad consultations with representatives from the private sector, NGOs, civil society, and the donor community. The SCA identifies priority sectors based on the potential for growth, export opportunities, and employment generation along with key constraints to development in each of these sectors. These sectors include agriculture and agro-industries, fisheries, tourism, art crafts and cultural industries, textile and clothing industries, and information and communication technologies and teleservices. Technical committees comprising representatives from the private and public sectors are in charge of defining the action plans for the implementation of the SCA.

18. The implementation of the SCA is expected to begin in June 2006, and its action plan to be aligned with the revised PRSP, which will reflect the key priorities of the government. The implementation of poverty reduction policies appears to be associated with a continuous decline in the number of poor households over recent years. Available estimates indicate that poverty declined from 57 percent in 2001 to 54 percent in 2004 .

\section{CONCLUSION}

19. As previously announced, my authorities are eager to maintain their close relation with the Fund at the expiry of the current arrangement under the PRGF. Major accomplishments were made in achieving key objectives under PRGF arrangements, including continued macroeconomic stability, sound financial system, and fiscal and debt sustainability. As we noted in a previous Board meeting on Senegal, the authorities' intention 
is to attain higher growth rates and reduce poverty further through a private sector-led economy. To achieve this objective, increased access to international capital markets is necessary and, to this end, the benefit of the Fund's signaling role is crucial. It is therefore the authorities' view that a nonfinancial arrangement will be more appropriate at this stage. We are hopeful that Directors will continue to provide their unfailing support to the authorities' future development endeavors.

20. In view of the major progress made in the implementation of the program and the corrective measures taken by the authorities, I would like to request Directors' support for the proposed decision. 